Darby Freitas de Albuquerque Lopes

\title{
Estimativa da Atitude e Posição e Controle Robusto de um Helicóptero Autônomo
}

Dissertação de Mestrado apresentada à Escola de Engenharia de São Carlos da Universidade de São Paulo, como parte dos requisitos para obtenção do título de Mestre em Ciências, Programa de Engenharia Elétrica

Área de Concentração:

Sistemas Dinâmicos

Orientador:

Prof. Dr. Marco Henrique Terra

São Carlos

2010 
AUTORIZO A REPRODUÇÃO E DIVULGAÇÃO TOTAL OU PARCIAL DESTE TRABALHO, POR QUALQUER MEIO CONVENCIONAL OU ELETRÔNICO, PARA FINS DE ESTUDO E PESQUISA, DESDE QUE CITADA A FONTE.

Ficha catalográfica preparada pela Seção de Tratamento da Informação do Serviço de Biblioteca - EESC/USP

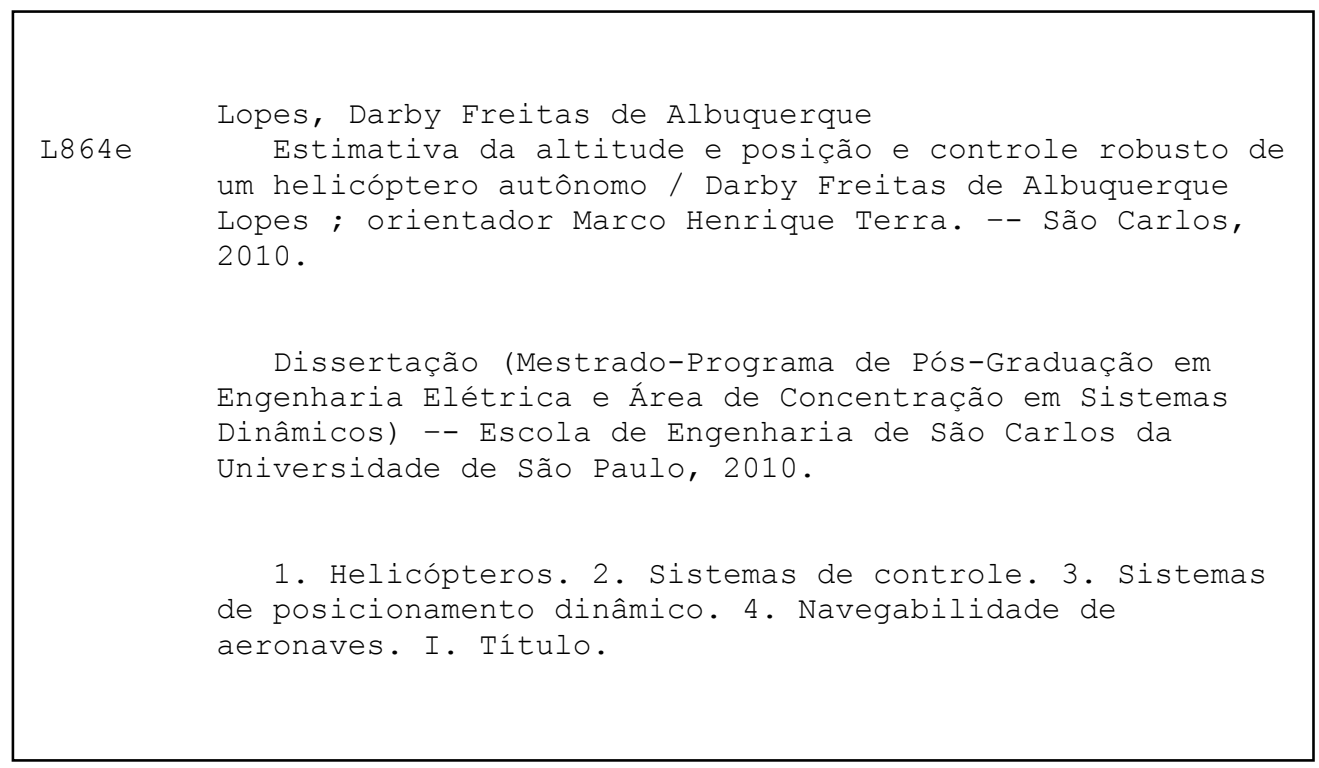




\section{FOLHA DE JULGAMENTO}

Candidato(a): Engenheiro Eletricista DARBY FREITAS DE ALBUQUERQUE LOPES.

Dissertação defendida e julgada em 10/12/2010 perante a Comissão Julgadora:
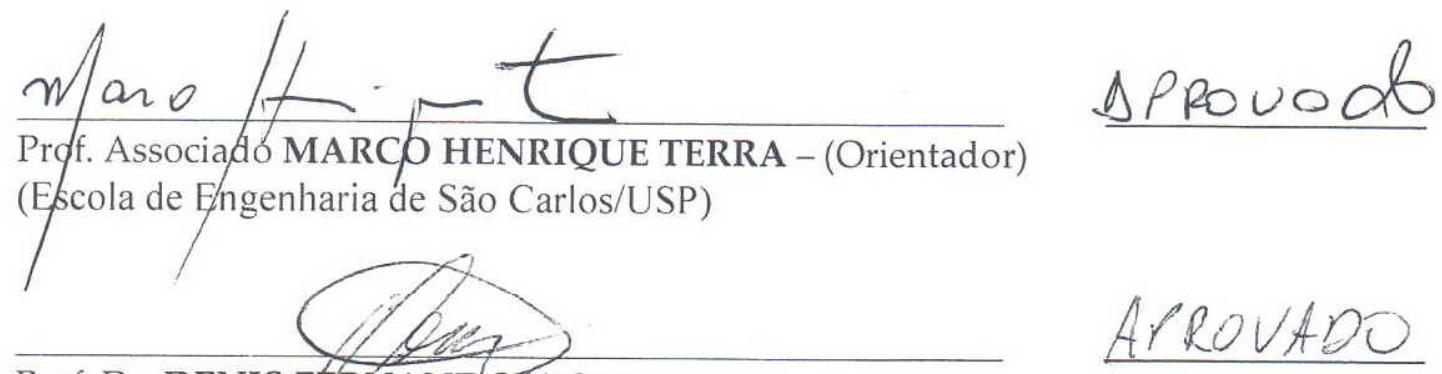

Prof. Dr. DENIS ERTNANDOWOLF

(Instituto de Ciências Matemáticas e de Computação/USP)

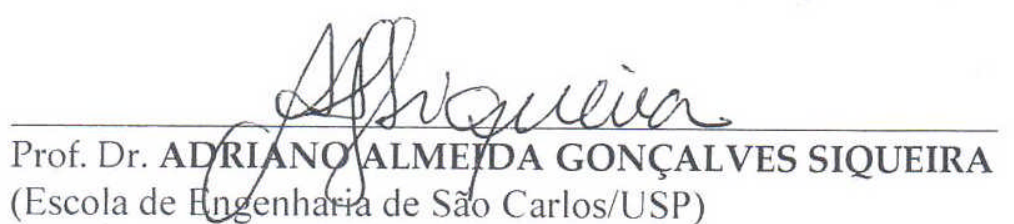

APROVADO

(Escola de Engenharia de São Carlos/USP)
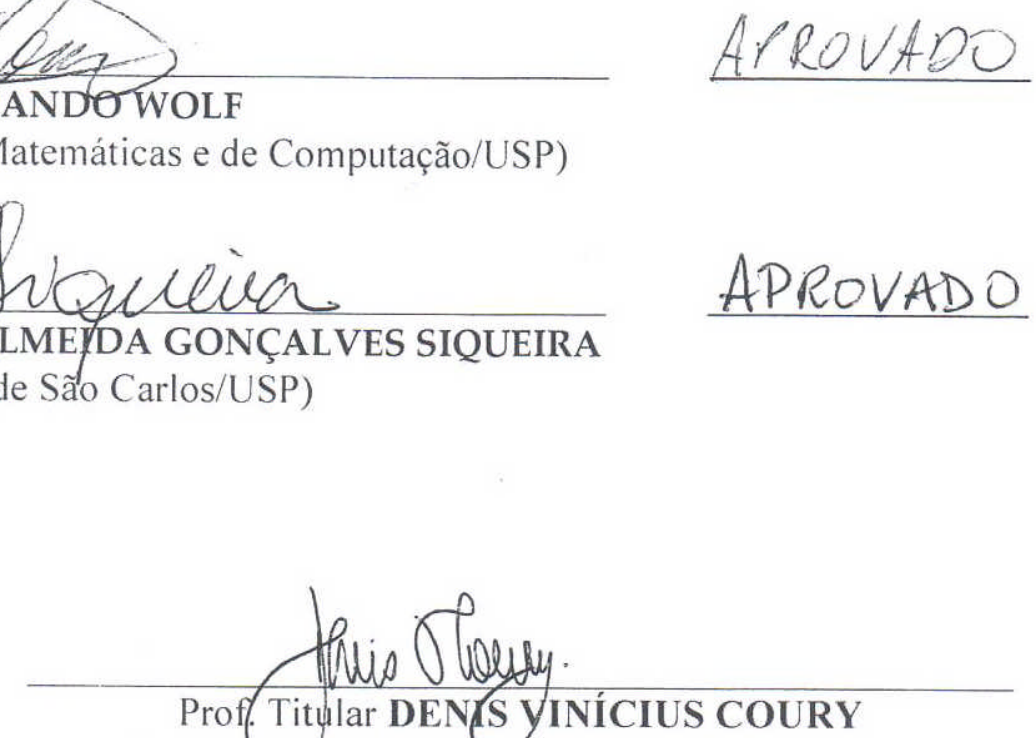

Coordenador do Programa de Pós-Graduação em

Engenharia Elétrica

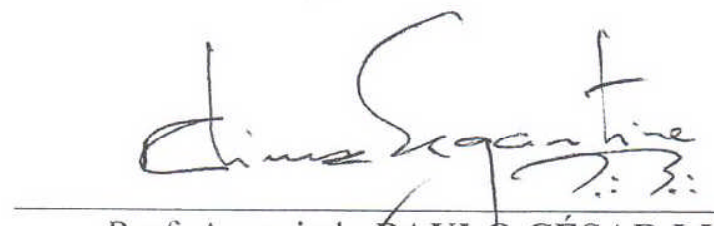

Prof. Associado PAUL $\varnothing$ CÉSAR LIMA SEGANTINE

Presidente da Comissão da Pós-Graduação da EESC 

Dedico este trabalho à minha esposa, Carla, companheira incomparável; aos meus pais, Átila e Antônia, modelos de honestidade, amor e perseverança; aos meus irmãos, Elohim e Eloha, por batalharem por seus sonhos; e ao meu sobrinho, Murilo, fonte de alegria inesgotável. 



\section{Agradecimentos}

Ao Todo-Poderoso, pela saúde e pela força concedidas durante o curso desta jornada.

À Carla, minha amada esposa, pelo companheirismo, amor e incentivo incessantes empregados durante esta empreitada.

Ao Prof. Dr. Marco Henrique Terra pela oportunidade, confiança, orientação e paciência dispensadas ao longo deste trabalho.

Ao Prof. Dr. Adriano Almeida Gonçalves Siqueira pela atenção, apoio e importantes contribuições para realização deste trabalho.

À instituição Universidade de São Paulo em São Carlos por toda a infraestruta oferecida.

Aos professores das disciplinas pela dedicação demonstrada e aos funcionários do Departamento de Engenharia Elétrica da USP São Carlos, Marisa e Jussara em especial, que de alguma forma, direta ou indiretamente, contribuíram com o trabalho.

Aos camaradas do Laboratório de Sistemas Inteligentes (LASI): Aline Bianco, Amanda Manfrim, Daniel Vaz, Gildson de Jesus, Gustavo Brito, Igor Breda, João Paulo Cerri, Leo Tubota, Miguel Vilca, Pedro Campos, Roberto Inoue, Samuel Lourenço e Tatiana Pazelli, pelas trocas de experiência, sugestões, confraternização, pelo companheirismo e respeito.

Aos colegas da turma Belém de Especialização em Gestão de Projetos FDC-Vale: André Ligório, Cadu Fois, Edan Rios, Francisco Fontenele, Gabriel Boamorte e Rannieri Barros, pelo incentivo na etapa de finalização desta dissertação.

À Fundação de Amparo à Pesquisa de São Paulo (FAPESP, processo 2007/06224-7) pela concessão da bolsa de mestrado e pelo apoio financeiro para a realização desta pesquisa.

E, finalmente, à minha amada família e aos meus grandes amigos que, mesmo distantes, demonstraram todo o amor e apoio para que eu seguisse em frente. 

"Há homens que lutam um dia e são bons. Há outros que lutam um ano e são melhores. Há os que lutam muitos anos e são muito bons. Porém, há os que lutam toda a vida. Esses são os imprescindiveis." 



\section{Resumo}

LOPES, D. F. de A.Estimativa da Atitude e Posição e Controle Robusto de um Helicóptero Autônomo. São Carlos, 2010, Dissertação (Mestrado) - Escola de Engenharia de São Carlos, Universidade de São Paulo.

Este trabalho aborda o estudo de um sistema de referência inercial de posição e atitude e um sistema de controle para um helicóptero autônomo utilizando, como base para formulação e testes, o modelo linearizado da aeronave Yamaha R-MAX. Um sistema de referência inercial (INS) e um sistema de referência de atitude e orientação (AHRS) são utilizados para estimar a posição e atitude da aeronave, e estimadores robustos baseados no filtro de Kalman são empregados para minimizar os efeitos de incertezas paramétricas. É utilizada uma estratégia de controle em cascata com três malhas consistindo de uma malha interna para garantir a estabilidade do helicóptero (são utilizadas as técnicas LQR e $\mathscr{H}_{\infty}$, separadamente), uma malha intermediária baseada em linearização por realimentação (FLC) para desacoplar os pares entrada/saída e uma malha externa baseada em um controlador proporcional-derivativo (PD) para permitir o rastreamento da trajetória. Resultados de simulação são apresentados para avaliar o desempenho de cada abordagem.

Palavras-Chave: Helicóptero, sistemas de controle, sistemas de posicionamento dinâmico, navegabilidade de aeronaves. 



\section{Abstract}

LOPES, D. F. de A.Attitude and Position Estimation and Robust Control of an Autonomous Helicopter. São Carlos, 2010, Dissertation (Master) - Escola de Engenharia de São Carlos, Universidade de São Paulo.

This work concerns the study of an inertial reference system and a control system for an autonomous helicopter using, as basis for the formulation and testing, the linearized model of the aircraft Yamaha R-MAX. An inertial navigation system (INS) and an attitude and orientation reference system (AHRS) are used to estimate the position and attitude of the aircraft and robust estimators based on Kalman filter are employed to minimize the effects of parametric uncertainties. A cascaded control architecture with three control methodologies is used, consisting of an inner-loop to ensure stability of the helicopter (the LQR and $\mathscr{H}_{\infty}$ techniques are used, separately), a mid-loop based on linearization feedback (FLC) to decouple the dynamics of the lateral, longitudinal, vertical and heading axes and an outer-loop based on a proportional-derivative (PD) controller to enable trajectory tracking. Simulation results are presented to evaluate the performance of each approach.

Keywords: Helicopter, control systems, dynamic positioning systems, aircraft navigability. 



\section{Lista de Figuras}

2.1 Sistemas de Coordenadas Referenciais: (a) inercial, (b) ECEF, (c) geográfico e $(d)$ do corpo [36]. . . . . . . . . . . . . . . . . . . . . . . . p. p. 29

2.2 Transformação do sistema de coordenada do corpo para o sistema de navegação [36]. . . . . . . . . . . . . . . . . . p. 31

$3.1 \quad$ Diagrama de blocos de um sistema de navegação [36]. . . . . . . . . . . . . p. p.33

$4.1 \quad$ Modelo do helicóptero em diagrama de blocos. . . . . . . . . . . . . . . . . p. p. 47

4.2 Yamaha R-MAX. . . . . . . . . . . . . . . . . . . . . . . . . . . . p. 49

$4.3 \quad$ Diagrama de blocos ilustrando as etapas do aplicativo CIFER. . . . . . . . . p. p.49

5.1 Diagrama de blocos do sistema. $\ldots \ldots \ldots \ldots \ldots$. . . . . . . . . . . . . . . .

6.1 Comparação entre a posição da aeronave e a saída do filtro de Kalman: (a) Emx, (b)Emy, (c)Emz. . . . . . . . . . . . . . . . . . p. 63

6.2 Comparação entre as atitudes e as saídas correspondentes do filtro de Kalman: (a) Rolagem, (b) Arfagem (c) Guinada. . . . . . . . . . . . . . . . . . p.63

6.3 Comparação entre a posição da aeronave e a saída da estimativa robusta A: (a) $\operatorname{Emx},($ b) $\operatorname{Em} y,(c) \operatorname{Em} z . \ldots \ldots$ p. 64

6.4 Comparação entre as atitudes e as saídas correspondentes da estimativa robusta A: (a) Rolagem, (b) Arfagem (c) Guinada. . . . . . . . . . . . . . . . . p.64

6.5 Comparação entre a posição da aeronave e a saída da estimativa robusta B: (a) Emx, (b)Emy, (c)Emz. . . . . . . . . . . . . . . . . . . p. 65

6.6 Comparação entre as atitudes e as saídas correspondentes da estimativa robusta B: (a) Rolagem, (b) Arfagem (c) Guinada. . . . . . . . . . . . . . . . . p. 65

6.7 Distúrbios externos. . . . . . . . . . . . . . . . . . . p. 66

6.8 Comparação entre a posição desejada da aeronave e a posição obtida, para distúbrio $\left[\begin{array}{lll}W_{x} & W_{y} & W_{z}\end{array}\right]=\left[\begin{array}{lll}2 & 1 & 1\end{array}\right]:$ (a) em $x,(b)$ em y e (c) em z. $\ldots . . . \quad$ p. 67 
$6.9 \quad$ Comparação entre a atitude da aeronave para o distúrbio $\left[\begin{array}{lll}W_{x} & W_{y} & W_{z}\end{array}\right]=$

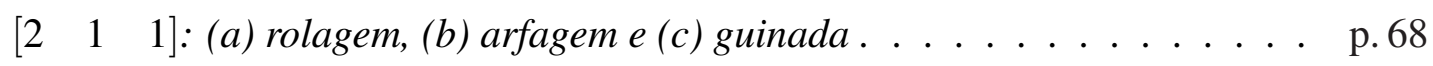

6.10 Variação da norma da matriz $A(\rho(t))$ durante o período de simulação. . . . . p. 69 


\section{Lista de Tabelas}

4.1 Características físicas do Yamaha R-MAX. $\ldots \ldots \ldots \ldots$. . . . . . . . p. 49

4.2 Elementos dos Vetores de Estado e de Entradas. . . . . . . . . . . . . p.50

6.1 Desempenho de cada filtro de acordo com a Equação (6.1). . . . . . . . . . . p. 63 



\section{Lista de Abreviaturas}

AHRS Sistema de Referência de Atitude e Orientação (Attitude and Heading Reference System, em inglês)

BDU Dados com Incertezas Limitadas (Bounded Data Uncertains, em inglês)

CIFER ${ }^{\circledR} \quad$ Comprehensive Identification from FrEquency Responses, em inglês

CNF Realimentação Não-linear Composta (Composite Nonlinear Feedback, em inglês)

DML Desigualdade Matricial Linear

ECEF Earth-Centered Earth-Fixed

EKF Filtro de Kalman Estendido (Extended Kalman Filter, em inglês)

ER Estimador Robusto

EWC Critério de Erro Branqueador (Error Whitening Criterion, em inglês)

FLC Controlador de Linearização por Realimentação (Feedback Linearization Controller, em inglês)

GPS Sistema de Posicionamento Global (Global Positional System, em inglês)

IMU Unidade de Medida Inercial (Inertial Measurement Unit, em inglês)

INS Sistema de Navegação Inercial (Inercial Navegation System, em inglês)

LLA Latitude, Longitude e Atitude

LMS Mínimo Quadrático Médio (Least Mean Square, em inglês)

LPV Linear a Parâmetros Variantes

LQR Regulador Quadrático Linear (Linear Quadratic Regulator, em inglês)

MEMS Sistema Micro-EletroMecânico (Micro Electro Mechanical System, em inglês) 
NED Norte, leste e para baixo (North, east and down, em inglês)

PD Proporcional-Derivativo

TLS Mínimo Quadrático Total (Total Least Square, em inglês)

UKF Uscented Kalman Filter, em inglês

VANT Veículo Aéreo Não-Tripulado

VTOL Decolagem e Pouso Verticais (Vertical Take Off and Landing, em inglês) 


\section{Sumário}

\begin{tabular}{lll}
\hline Introdução & p. 23
\end{tabular}

1.1 Motivação e Trabalhos Anteriores $\ldots \ldots \ldots \ldots$. . . . . . . . . . 23

1.2 Organização da Dissertação $\ldots \ldots \ldots \ldots \ldots$. . . . . . . . . . . . 26

\begin{tabular}{|ll}
2 Sistemas de Coordenadas Referenciais & p. 27
\end{tabular}

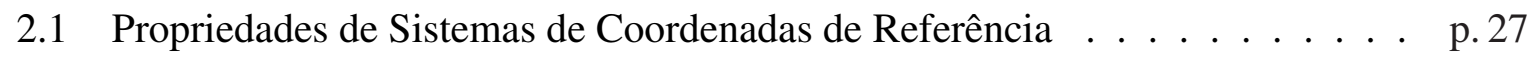

2.2 Definições de Sistemas de Coordenadas de Referência $\ldots \ldots \ldots$. . . . . . . p. 28

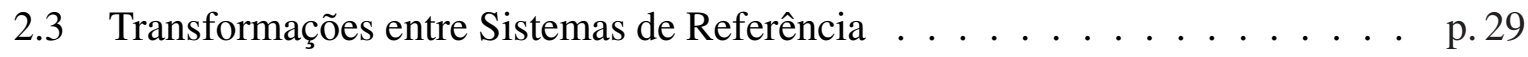

2.3.1 Transformação: ECEF para Geográfico . . . . . . . . . . . . . . . p. 29

2.3.2 Transformação: Sistema do Corpo para Sistema Geográfico . . . . p. p.30

\begin{tabular}{|ll}
\hline Sistema de Navegação & p. 33
\end{tabular}

3.1 Sistema de Referência de Atitude e Orientação $\ldots \ldots$. . . . . . . . . . . p. 34

3.1 .1 Modelo em Espaço de Estado . . . . . . . . . . . . . . . p. 34

3.2 Sistema de Navegação Inercial $\ldots \ldots \ldots \ldots$. . . . . . . . . . . . . 36

3.2.1 Modelo em Espaço de Estado . . . . . . . . . . . . . . . p. 37

3.3 Estimativa Robusta BDU . . . . . . . . . . . . . . . . . . . . . p. 39

3.4 Estimativa Ótima Robusta $\ldots \ldots \ldots \ldots \ldots \ldots \ldots$. . . . . . . . . . . .

4 Modelo Dinâmico do Helicóptero $\quad$ p. 45

4.1 Visão Geral $\ldots \ldots \ldots \ldots \ldots \ldots \ldots \ldots \ldots \ldots$. . . . . . . . . . . . . . . . . . .

4.2 Forças, Momentos e Equações Cinemáticas $\ldots \ldots \ldots$. . . . . . . . . . p.47

4.3 Modelo Yamaha $R$-MAX Adaptado $\ldots \ldots \ldots \ldots \ldots$. . . . . . . . . . . . 48 
5 Sistema de Controle $\quad$ p. 53

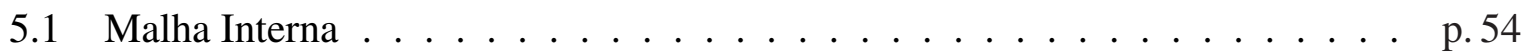

$5.1 .1 \quad$ Regulador Quadrático Linear . . . . . . . . . . . . . . . . . . p. 54

5.1 .2 Controlador $\mathscr{H}_{\infty}$ não-linear $\ldots \ldots \ldots \ldots$. . . . . . . . . . . . . . . .

5.2 Malha Intermediária - Controlador de Linearização por Realimentação ～ p.58

5.3 Malha Externa - Controlador PD . . . . . . . . . . . . . . . . . p. 59

5.4 Lei de Controle em Cascata $\ldots \ldots \ldots \ldots$. . . . . . . . . . . . . . . p. 59

5.5 Seguidor de Waypoints em Sistema de Coordenadas do Corpo . . . . . . . . p. 59

$\begin{array}{lll}6 & \text { Resultados } & \text { p. } 61\end{array}$

6.1 Comparação entre Filtros $\ldots \ldots \ldots \ldots$. . . . . . . . . . . . 61

6.2 Comparação entre Sistemas de Controle da Malha Interna . . . . . . . . . . . p. 63

\begin{tabular}{lll}
\hline 7 & Conclusão & p. 71
\end{tabular}

\begin{tabular}{ll}
\hline Referências Bibliográficas & p. 73
\end{tabular}

\begin{tabular}{ll}
\hline Apêndice A - Quatérnios & p. 77
\end{tabular}

A.1 Considerações geométricas . . . . . . . . . . . . . . . p. 80

A.2 Operador quatérnio de rotação $\ldots \ldots \ldots \ldots \ldots$. . . . . . . . . 81

A.3 Conversão entre quatérnios e ângulos de Euler $\ldots \ldots \ldots$. . . . . . . . . p. 82

A.4 Derivada do quatérnio . . . . . . . . . . . . . . . . . . p. 84

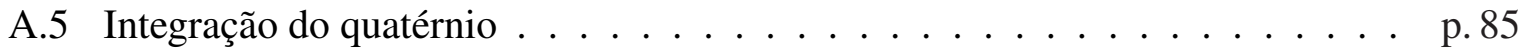




\section{$1 \quad$ Introdução}

\subsection{Motivação e Trabalhos Anteriores}

Helicópteros autônomos são equipamentos de alta tecnologia que devem satisfazer os mais altos padrões industriais de qualidade técnica e confiabilidade. Com a crescente disponibilidade de recursos computacionais de alto desempenho, avanços em tecnologias de transmissão de dados e posicionamento global, os custos dos veículos aéreos não-tripulados (VANTs) têm diminuído e, desta forma, o interesse da comunidade científica em VANTs aumentou significativamente. Esses aspectos também têm permitido o desenvolvimento de veículos cada vez mais confiáveis e versáteis.

Por serem capazes de realizar uma grande variedade de manobras como, por exemplo, decolagem e pouso vertical, voo longitudinal/lateral, piruetas e ainda planar eficientemente por longos períodos de tempo, eles estão aptos a executar missões complexas em diversos campos de aplicação, civil ou militar. Estas características os fazem importantes para pesquisas terrestres, aplicações em engenharia, vigilância e monitoramento, operações de resgate e limpeza de locais perigosos, tornando-os indispensáveis em várias aplicações onde intervenção humana é considerada difícil ou perigosa.

A construção de um helicóptero autônomo completamente funcional exige o estudo aprofundado de alguns componentes essenciais. Entre eles estão a plataforma de hardware, o projeto e integração de um sistema de software, a modelagem da aerodinâmica, e projeto e implementação das leis de controle de voo [1].

Um dos desafios inerentes ao desenvolvimento destes helicópteros diz respeito à sua modelagem matemática. Há, na literatura, uma grande variedade de trabalhos que abordam diferentes métodos usados para descrever o comportamento dinâmico da aeronave. Um deles é utilizar um modelo linear em espaço de estado nos modos pairar e voo de cruzeiro, como feito por Cheng et al. [2] para o helicóptero Yamaha R-Max, obtido a partir de dados de teste de voo utilizando um método de identificação de sistema no domínio da frequência. Gavrilets et al. [3] desenvolve- 
ram um modelo dinâmico não-linear de pequena ordem de um helicóptero acrobático miniatura, usando expressões analíticas simplificadas para os componentes de força e momento. Uma modelagem não-linear de um helicóptero de pequena escala também foi proposta por Beckmann e Borges [4], eles identificaram seus trinta e dois parâmetros utilizando métodos de minimização do erro de predição. Em [5], Zhou aplicou EWC (Error Whitening Criterion, em inglês) na identificação de sistema para o movimento de decolagem de um helicóptero de fabricação própria e comparou o resultado do EWC-LMS com o LMS (Least Mean Square, em inglês) e TLS (Total Least Square, em inglês), destacando o melhor desempenho do EWC-LMS.Um novo método de identificação de sistema para pequenos VANTs combinando um estimador UKF (Uscented Kalman Filter, em inglês) e um identificador baseado em redes neurais artificiais foi apresentado por [6]. Este método foi aplicado a um VANT de asas fixas e a um de asas rotativas.

Diversas instituições espalhadas pelo mundo possuem projetos visando à construção de helicópteros autônomos. Amidi e Miller [7] apresentaram uma visão geral do projeto do helicóptero autônomo do Instituto de Robótica da Universidade Carnegie Mellon, com foco nas seguintes capacidades: estabilidade baseada em visão e controle; decolagem, seguimento de trajetória e pouso autônomos; mapeamento aéreo; e reconhecimento e manipulação de objetos. Um sistema de gerenciamento de voo implementado como inteligência embarcada em um VANT de asas rotativas foi proposto por Kim et al. [8]. Entre outras coisas, derivaram um modelo dinâmico não-linear no qual uma camada de controle de seguimento foi projetada utilizando controle preditivo não-linear integrado a um gerador de trajetória para planejamento logístico de ação. Em [9], Gavrilets et al. apresentaram o sistema aviônico, projeto do estimador de estado e o isolador de vibração para um helicóptero Xcell-60, do MIT, para desempenhar manobras agressivas de forma autônoma. Garcia e Valavanis [10] discutem os aspectos técnicos de um projeto de um pequeno helicóptero autônomo como um veículo de testes a fim de encorajar mais instituições científicas a trabalharem com tal tema, já que seu desenvolvimento é de longa duração e de alto custo.

Um sistema automático de controle de voo é essencial em missões de um helicóptero com mínima ou nenhuma interferência humana. Há, na literatura, muitos trabalhos que propõem controladores para movimento e trajetória de um helicóptero autônomo. Shim et al. [11] compararam três estratégias de controle: controle linear robusto multivariável, controle com lógica fuzzy com ajuste evolucionário e controle por rastreamento não-linear. Um método de controle através de equação de Lyapunov utilizando técnicas de backstepping foi proposto por Mahony, Hamel e Dzul [12]. Isidori, Marconi e Serran projetaram controladores não-lineares combinados com adaptação não-linear de saída regulável e estabilização robusta para o controle do movimento vertical de um helicóptero [13]. Em [14], foram projetados controladores robustos 
baseados em critérios $\mathscr{H}_{\infty}$, síntese $\mu$ e sistemas lineares a parâmetros variantes (LPV) para um helicóptero autônomo em voo longitudinal. Em [15], foi apresentada uma abordagem para controle de voo para um modelo dinâmico não linear de um helicóptero de seis graus de liberdade utilizando equação de Riccati dependente do estado cujos resultados experimentais foram mostrados utilizando um helicóptero X-Cell-90 e um Yamaha R-Max. Em [16], foi proposta uma metodologia na qual controladores baseados em redes neurais são envolvidos em uma simulação usando um modelo dinâmico qualitativamente similar a um helicóptero. Saripalli e Sukhatme [17] propuseram um controle ótimo de trajetória para aterrissagem de um helicóptero sobre um alvo em movimento, em que tentativas iniciais em simulação foram apresentadas. Em [18], um controle de posição e direção em cascata, levando em consideração o acoplamento dos quatro eixos de controle da aeronave em questão, foi proposto. Marconi e Naldi [19] projetaram um controlador não-linear, obtido pela combinação apropriada de ações de controle feedforward e high-gain e leis de realimentação de saturação associadas. Tais leis obtêm sucesso em reforçar as trajetórias desejadas de maneira robusta com respeito às incertezas caracterizando os parâmetros físicos e aerodinâmicos do helicóptero. Em [20], são apresentados o projeto e implementação de uma lei de controle para voo autônomo para um helicóptero de pequena escala, incorporando uma técnica de realimentação não-linear composta (CNF) (Composite Nonlinear Feedback, em inglês) seguida de inversão dinâmica. Outros trabalhos envolvendo controle de helicópteros autônomos são encontrados em [21, 22, 23, 24, 25, 26, 27, 28].

Outra etapa importante no desenvolvimento de um helicóptero autônomo diz respeito ao sistema de navegação, que pode ser definido como o sistema que estima o estado da aeronave (posição, velocidade e atitude) em tempo real enquanto o mesmo manobra ao longo de uma trajetória. Para a maioria das aplicações externas, os sensores de navegação incluem um sistema de medida inercial (IMU, em inglês), sistema de posicionamento global (GPS), uma bússola magnética e um algoritmo de estimação sofisticado [1].

Em [29], foram investigados três algoritmos de filtro de Kalman adaptativo que podem ser utilizados para aprimorar a estimativa das propriedades estocásticas de um sistema inercial de navegação de baixo custo, usando um sensor micro-eletromecânico (MEMS) IMU da Crossbow integrado com um GPS. Em [30], é descrito um sistema de determinação de atitude baseado em acelerômetros e em taxas de giroscópio utilizando filtro de Kalman extendido. Em [31], é abordado o problema da restituição da atitude e direção de um VANT VTOL, decolagem e pouso verticais (Vertical Take Off and Landing, em inglês), propondo uma estratégia de observação para restaurar a atitude partindo de uma medição da matriz de orientação e leituras de taxas angulares que sofrem interferência de um vetor de viés desconhecido. Abdelkrim et al. [32] utilizaram uma técnica de filtragem não-linear robusta baseada na teoria de controle $\mathscr{H}_{\infty}$ para 
sistema de navegação e posição a fim de se evitar problemas que técnicas clássicas sofrem como inicialização e os erros de linearização que degradam severamente o desempenho dos estimadores de localização de VANTs. Em [33], é proposto um novo algoritmo de filtragem fuzzy auto adaptativo de segunda ordem para sistema de navegação GPS/INS, sistema de navegação inercial (Inercial Navegation System, em inglês), a fim de diminuir os erros de navegação e aperfeiçoar o desempenho anti-interferência.

Como os sistemas robóticos podem estar sujeitos a perturbações externas e incertezas paramétricas, a estimativa robusta dos estados envolvidos é bastante útil para atenuar os efeitos indesejáveis dessas variáveis. O filtro de Kalman se apresenta como uma das ferramentas mais utilizadas para resolver problemas de filtragem recursiva. Uma premissa central na teoria do filtro de Kalman é a necessidade de que o modelo utilizado seja exato. Caso essa hipótese seja violada, o desempenho do filtro pode se deteriorar ou até se tornar instável.

Este trabalho aborda o estudo de um sistema de referência inercial de posição e atitude para um helicóptero não-tripulado utilizando fusão de sensores através de filtragem. Serão aplicados filtros robustos para minimizar os efeitos das incertezas nas estimativas dos estados de um helicóptero autônomo modelo Yamaha R-MAX. Os filtros implementados são os desenvolvidos em [34], que propôs um sistema para estimar modelos incertos no espaço de estado, e em [35], que pode ser tratado como uma abordagem mais abrangente que a primeira. $\mathrm{O}$ desempenho de cada configuração será comparado entre si e com uma formulação baseada no filtro de Kalman nominal. Será utilizado também o controlador em cascata apresentado em [18], combinando três metodologias de controle: regulador linear quadrático, controlador baseado em linearização por realimentação e um controle proporcional-derivativo. Será empregado um controlador $\mathscr{H}_{\infty}$ não-linear para estabilizar a planta e seu desempenho será comparado com o regulador linear quadrático.

\subsection{Organização da Dissertação}

Esta dissertação está dividida em seis Capítulos. No Capítulo 2, são discutidos os sistemas de coordenadas de referência utilizados em sistemas de navegação e as relações matemáticas pertinentes. O Capítulo 3 apresenta o sistema de navegação inercial, o sistema de referência de atitude e orientação, e os filtros utilizados para estimar os estados. No Capítulo 4, é apresentado o modelo matemático de um helicóptero. O Capítulo 5 aborda as estratégias de controle empregadas neste trabalho. O Capítulo 6 apresenta os resultados de simulações obtidos durante a execução deste projeto. Finalmente, o Capítulo 7 apresenta as conclusões e contribuições. 


\section{Sistemas de Coordenadas Referenciais}

Uma parte importante no estudo dos sistemas de navegação é a correta utilização dos sistemas de referência bem como a transformação de quantidades medidas e calculadas entre os vários sistemas de referência. Este capítulo trata da definição de alguns sistemas de coordenadas de referência e dos métodos para transformação das coordenadas dos pontos e representação de vetores entre eles.

\subsection{Propriedades de Sistemas de Coordenadas de Referência}

Os sistemas de navegação envolvem medições de variáveis em diversos sistemas de referência. Para garantir a adequada comunicação e interoperabilidade entre todos que trabalham com o sistema, cada sistema referencial e suas propriedades devem ser claramente definidos [36].

A menos que seja dito o contrário, assume-se que todos os sistemas coordenados de referência retangulares possuem três eixos definidos como ortonormais e seguindo a regra da mão direita.

Devido aos efeitos gravitacionais próximos à superfície da Terra, é geralmente conveniente considerar sistemas de coordenadas elipsoidais além dos sistemas de coordenadas retangulares. Considera-se, portanto, a utilização do geóide da Terra, que é uma superfície hipoteticamente equipotencial do campo de gravidade terrestre que coincide com o nível médio do mar.

Em todo o texto, vetores e matrizes serão escritas em negrito. Quando um vetor for representado relativo a um sistema de referência específico, este será indicado por um sobrescrito. Por exemplo, $\omega^{a}$ é o vetor $\omega$ representado com respeito ao sistema de referência $a$. A matriz de rotação para transformar um vetor representado em um sistema $a$ para um sistema $b$ é escrita como $\mathbf{R}_{a}^{b}$ em que

$$
\omega^{b}=\mathbf{R}_{a}^{b} \omega^{a}
$$




\subsection{Definições de Sistemas de Coordenadas de Referência}

Um sistema de coordenadas de referência inercial é um sistema de referência no qual as leis de Newton se aplicam. Um sistema inercial é, portanto, não acelerado, mas pode estar em movimento linear uniforme. Sua origem é arbitrária e os eixos coordenados podem apontar para quaisquer direções mutuamente perpendiculares. Todos os sensores inerciais produzem medidas relativas a um referencial inercial, resolvidos ao longo do eixo sensível do instrumento.

O sistema de coordenadas de referência ECEF (Earth-centered, Earth-fixed) tem sua origem fixada no centro da Terra e utiliza dois sistemas de coordenadas diferentes para descrever a localização de um ponto: o sistema de coordenadas retangulares $\left((x, y, z)^{e}\right)$ e o sistema de coordenadas geodésicas $\left((\phi, \lambda, h)^{e}\right.$, latitude, longitude e altura, respectivamente). O índice 'e' se refere ao sistema de coordenadas ECEF. Devido à rotação da Terra, o sistema ECEF não é um sistema de referencial inercial.

O sistema de coordenadas de referência geográfico, ou de navegação, possui poucos pontos que o diferem de outros sistemas, sendo um deles o fato de sua origem se mover com o veículo e ser a projeção da origem do sistema de coordenadas do veículo sobre o elipsóide de referência. A posição do veículo neste sistema é $\mathbf{x}^{g}=[0,0,-h]^{T}$, em que a latitude $\phi$ e a longitude $\lambda$ definem a posição da origem no elipsóide de referência. O outro ponto de distinção é que $\frac{d}{d t} \mathbf{x}^{g}=[0,0,-\dot{h}]^{T}$ não representa o vetor velocidade do veículo, mas é obtido a partir de uma transformação do vetor velocidade do veículo no sistema de referência ECEF.

O sistema de referência do corpo é rigidamente acoplado ao veículo de interesse, geralmente em um ponto fixo como o centro de gravidade. Escolher o centro de gravidade como sua origem simplifica a derivação das equações cinemáticas e é geralmente conveniente para projeto de sistemas de controle. O eixo $u$ é definido na direção avante do veículo. O eixo $w$ é definido como apontando para a parte inferior do veículo e o eixo $v$ completa o sistema coordenado ortogonal pela regra da mão direita. As direções dos eixos definidos não são únicas, mas são típicas em aplicações de veículos aéreos e submarinos. O sistema de referência do corpo não é um sistema de referência inercial.

As Figuras 2.1(a), 2.1(b), 2.1(c) e 2.1(d) exibem os sistemas de coordenadas referenciais inercial, ECEF, geográfico e do corpo, respectivamente. Na Figura 2.1(d), $p$ é a velocidade angular em torno do eixo $u$ (taxa de rolagem), $q$ é a velocidade angular em torno do eixo $v$ (taxa de arfagem), e $r$ é a velocidade angular em torno do eixo $w$ (taxa de guinada). 


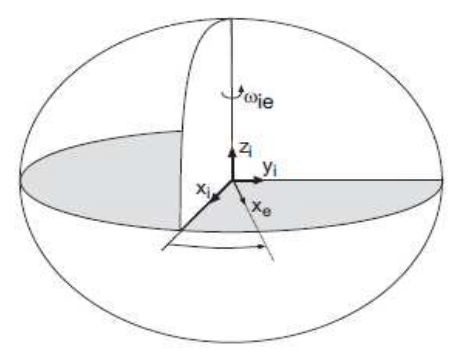

(a)

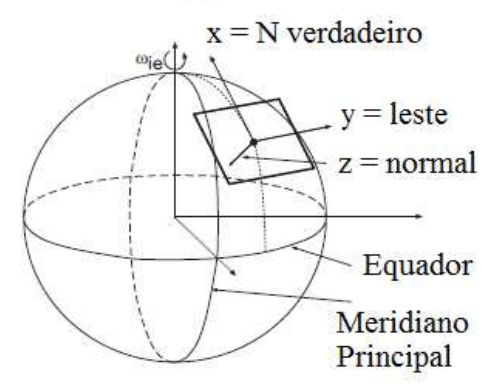

(c)

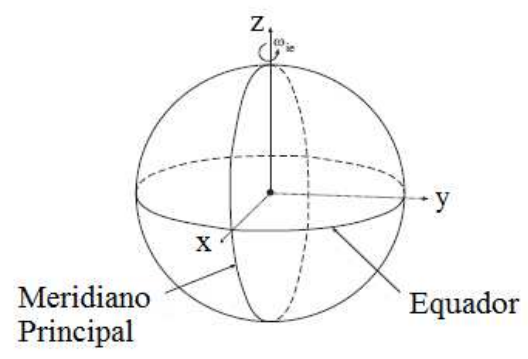

(b)

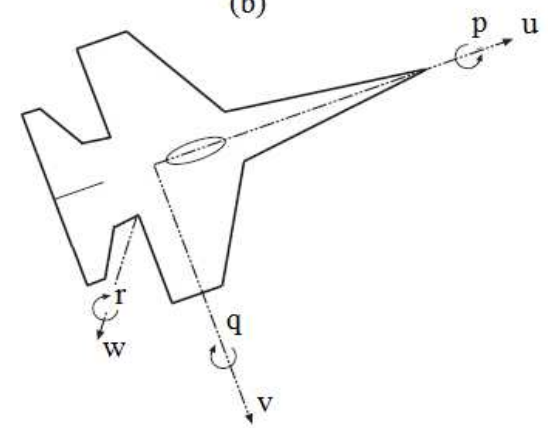

(d)

Figura 2.1: Sistemas de Coordenadas Referenciais: (a) inercial, (b) ECEF, (c) geográfico e (d) do corpo [36].

\subsection{Transformações entre Sistemas de Referência}

Esta seção apresenta as operações de transformação entre sistemas de coordenadas de referência úteis nas aplicações de navegação.

\subsubsection{Transformação: ECEF para Geográfico}

O vetor $\mathbf{v}_{e}^{g}=\left[v_{n}, v_{e}, v_{d}\right]^{T}$ representa os componentes norte, leste e para baixo (NED, north, east e down, em inglês) do vetor velocidade ao longo dos eixos instantâneos do sistema de referência geográfico e o vetor $[\dot{\phi}, \dot{\lambda}, \dot{h}]^{e}$ contém os componentes do vetor velocidade em coordenadas geodésicas do sistema de referência ECEF. A Equação (2.2) apresenta a relação de transformação entre os dois vetores:

$$
\mathbf{v}_{e}^{g}=\left[\begin{array}{c}
v_{n} \\
v_{e} \\
v_{d}
\end{array}\right]=\left[\begin{array}{ccc}
\left(R_{M}+h\right) & 0 & 0 \\
0 & \left(R_{N}+h\right) \cos (\phi) & 0 \\
0 & 0 & -1
\end{array}\right]\left[\begin{array}{c}
\dot{\phi} \\
\dot{\lambda} \\
\dot{h}
\end{array}\right],
$$


sua relação inversa é facilmente obtida por

$$
\left[\begin{array}{c}
\dot{\phi} \\
\dot{\lambda} \\
\dot{h}
\end{array}\right]=\left[\begin{array}{ccc}
\frac{1}{R_{M}+h} & 0 & 0 \\
0 & \frac{1}{\left(R_{N}+h\right) \cos \phi} & 0 \\
0 & 0 & -1
\end{array}\right]\left[\begin{array}{c}
v_{n} \\
v_{e} \\
v_{d}
\end{array}\right]
$$

onde $R_{M}(\phi)$ é chamado de raio meridional ou raio da elipse, $R_{N}(\phi)$ é chamado de raio de curvatura da primeira vertical ou grande normal, dados por:

$$
\begin{aligned}
& R_{M}(\phi)=\frac{a\left(1-e^{2}\right)}{\left(1-e^{2} \operatorname{sen}^{2}(\phi)\right)^{\frac{3}{2}}} \\
& R_{N}(\phi)=\frac{a}{\left(1-e^{2} \operatorname{sen}^{2}(\phi)\right)^{\frac{1}{2}}} .
\end{aligned}
$$

sendo $a$ o raio equatorial e $e$ a excentricidade, cujos valores são $6.378 .137 m$ e 0,08181919 , respectivamente, segundo o sistema geodésico WGS84.

\subsubsection{Transformação: Sistema do Corpo para Sistema Geográfico}

Os eixos do sistema de referência geográfico são indicados pelos vetores unitários $I, J \mathrm{e}$ $K$ que, inicialmente, estão alinhados à direção do sistema referencial tangente $(n, e, d)$ e a transformação do sistema do corpo para o sistema geográfico é obtida através de três rotações. A Figura 2.2a exibe o resultado da rotação de guinada num ângulo $\psi$ radioanos, gerando os vetores unitários $I^{\prime}, J^{\prime}$ e $K^{\prime}=K$. A Figura $2.2 \mathrm{~b}$ mostra a rotação do sistema obtido anteriormente num ângulo $\theta$ radianos sobre o eixo $J^{\prime}$, gerando $I^{\prime \prime}, J^{\prime \prime}=J^{\prime}$ e $K^{\prime \prime}$, alinhando o vetor unitário $I^{\prime \prime}$ com o eixo- $u$ do veículo. E finalmente, a Figura 2.2 r exibe a rotacão do sistema anterior de um ângulo $\phi$ radianos alinhando os novos eixos $I^{\prime \prime \prime}=I^{\prime \prime}, J^{\prime \prime \prime}$ e $K^{\prime \prime \prime}$ aos eixos $u, v$ e $w$ do veículo, respectivamente.

A relação entre vetores pode ser definida por uma série de três rotações planares envolvendo os ângulos de Euler $[\phi]_{1}[\theta]_{2}[\psi]_{3}$ :

$$
\begin{aligned}
& \mathbf{v}^{b}=\left[\begin{array}{ccc}
1 & 0 & 0 \\
0 & c \phi & s \phi \\
0 & -s \phi & c \phi
\end{array}\right]\left[\begin{array}{ccc}
c \theta & 0 & -s \theta \\
0 & 1 & 0 \\
s \theta & 0 & c \theta
\end{array}\right]\left[\begin{array}{ccc}
c \psi & s \psi & 0 \\
-s \psi & c \psi & 0 \\
0 & 0 & 1
\end{array}\right] \mathbf{v}^{g} \\
& \mathbf{v}^{b}=\left[\begin{array}{ccc}
c \psi c \theta & s \psi c \theta & -s \theta \\
-s \psi c \phi+c \psi s \theta s \phi & c \psi c \phi+s \psi s \theta s \phi & c \theta s \phi \\
s \psi s \phi+c \psi s \theta c \phi & -c \psi s \phi+s \psi s \theta c \phi & c \theta c \phi
\end{array}\right] \mathbf{v}^{g} \\
& \mathbf{v}^{b}=\mathbf{R}_{g}^{b} \mathbf{v}^{g} \text {. }
\end{aligned}
$$




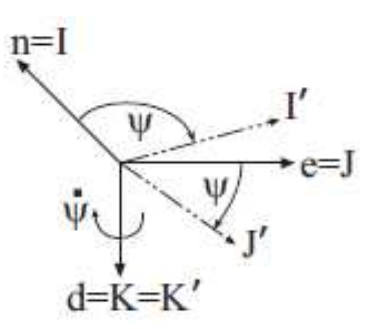

(a)

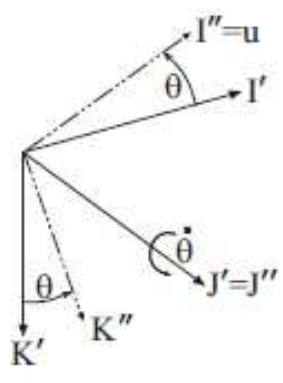

(b)

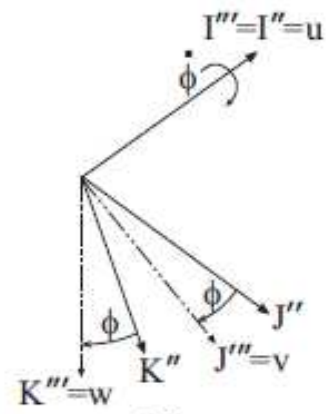

(c)

Figura 2.2: Transformação do sistema de coordenada do corpo para o sistema de navegação [36].

onde $\phi$ representa ângulo de rolagem, $\theta$ representa ângulo de arfagem e $\psi$ representa ângulo de guinada, $c x=\cos (x)$ e $s x=\operatorname{sen}(x)$. A operação inversa é

$$
\begin{aligned}
\mathbf{v}^{g} & =\mathbf{R}_{b}^{g} \mathbf{v}^{b} \\
& =\left(\mathbf{R}_{g}^{b}\right)^{T} \mathbf{v}^{b} .
\end{aligned}
$$

Uma vez que a sequência de rotações (neste caso, $z y x$ ) for especificada, a sequência de rotação angular para representar uma dada orientação rotacional relativa é única, exceto em pontos de singularidade. Por exemplo, para a sequência de rotações $z y x$, a sequência rotacional $[x]_{1}\left[\frac{\pi}{2}\right]_{2}[x]_{3}$ resulta na mesma orientação para qualquer $\|x\| \leq \pi$. Isto demonstra que a sequência de rotações zyx é singular nos pontos $\theta= \pm \frac{\pi}{2}$. Estes são os únicos pontos de singularidade desta sequência de rotações. Parametrizações livres de singularidade, como quatérnios (ver Apêndice (A), oferecem alternativas atraentes.

Quando a matriz $\mathbf{R}_{b}^{g}$ é conhecida, os ângulos de Euler podem ser determinados, para propósitos de controle ou planejamento, pelas seguintes equações:

$$
\begin{aligned}
\theta & =-\arctan \left(\frac{\mathbf{R}_{b}^{g}[3,1]}{\sqrt{1-\left(\mathbf{R}_{b}^{t}[3,1]\right)^{2}}}\right), \\
\phi & =\operatorname{atan} 2\left(\mathbf{R}_{b}^{g}[3,2], \mathbf{R}_{b}^{g}[3,3]\right), \\
\psi & =\operatorname{atan} 2\left(\mathbf{R}_{b}^{g}[2,1], \mathbf{R}_{b}^{g}[1,1]\right)
\end{aligned}
$$

em que $\operatorname{atan} 2(y, x)$ é uma função tangente inversa do quarto quadrante e os números nos colchetes se referem a um elemento específico da matriz. Por exemplo, $\mathbf{A}[i, j]=a_{i j}$ é o elemento 
na $i$-ésima linha e $j$-ésima coluna da matriz $\mathbf{A}$.

Maiores detalhes sobre estes e outros sistemas de referência bem como as transformações entre eles podem ser encontrados em [36]. 


\section{Sistema de Navegação}

Este capítulo apresenta as ferramentas necessárias para resolver o problema de localização e atitude de um helicóptero não tripulado. Serão utilizados um sistema de navegação inercial (INS) e um sistema de referência de atitude e orientação (AHRS). Em aplicações AHRS/GPS altamente acoplados, o modelo de estado do sistema é linear, contudo, o modelo de medição contém componentes que são não-lineares por natureza. Portanto é necessário o projeto de um filtro EKF (sigla em inglês para Filtro de Kalman Estendido) em tempo real, abordando os componentes não-lineares de acordo com a teoria de expansão de Taylor [37]. Assumindo que incertezas paramétricas possam estar presentes, casos em que o modelo utilizado para representar o sistema não é exato o suficiente, serão considerados projetos de dois filtros robustos distintos, propostos por [34] e [35], para lidar com este problema.

A Figura 3.1 exibe um diagrama de blocos ilustrando o sistema de navegação que será descrito neste Capítulo.

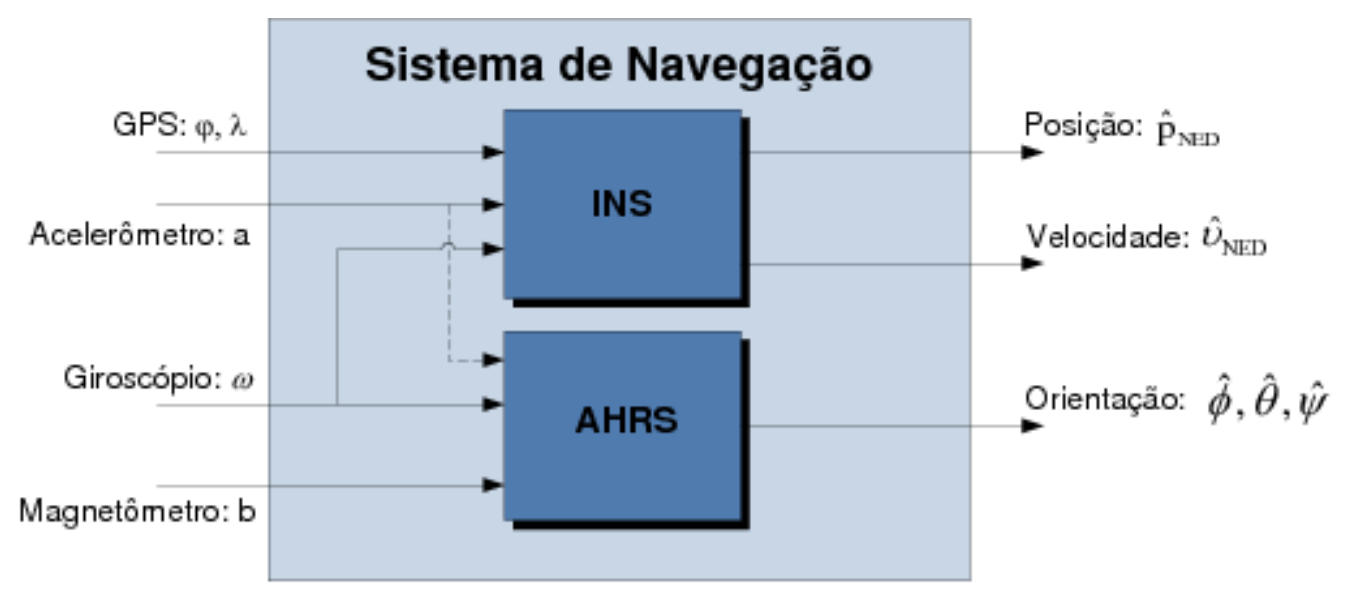

Figura 3.1: Diagrama de blocos de um sistema de navegação [36]. 


\subsection{Sistema de Referência de Atitude e Orientação}

Um sistema de referência de atitude e orientação é uma combinação de instrumentos capaz de manter uma estimativa precisa dos ângulos de rolagem $\phi$, arfagem $\theta$ e guinada $\psi$ do veículo enquanto o mesmo manobra [36]. As medidas dos acelerômetros e magnetômetros no sistema de coordenadas do corpo são transformadas para o sistema de navegação para predizer os vetores de aceleração gravitacional e campo magnético terrestre. Um filtro de Kalman utiliza medições residuais para estimar o erro de atitude e os fatores de calibração do sensor. Como o acelerômetro no sistema de coordenadas de referência do corpo mede especificamente a acelereração cinemática menos a aceleração gravitacional, o projeto será feito de modo a detectar quando o veículo estiver acelerando e ponderar ou ignorar as medições do acelerômetro durante esse período.

\subsubsection{Modelo em Espaço de Estado}

Nesta seção, quatérnios são utilizados para obter uma representação de atitude de um corpo rígido livre de singularidades. Utilizando as equações contidas no Apêndice $\mathrm{A}$, a derivada do quatérnio pode ser escrita matricialmente como

$$
\dot{q}=\frac{1}{2} q \otimes\left[\begin{array}{c}
0 \\
\boldsymbol{\omega}
\end{array}\right]=\frac{1}{2} \Omega(\boldsymbol{\omega}) q=\frac{1}{2} \Xi(q) \boldsymbol{\omega},
$$

sendo

$$
\Omega(\boldsymbol{\omega})=\left[\begin{array}{cc}
0 & -\boldsymbol{\omega}^{T} \\
\boldsymbol{\omega} & -\left[\boldsymbol{\omega}^{\times}\right]
\end{array}\right], \Xi(q)=\left[\begin{array}{c}
-\boldsymbol{q}^{T} \\
I_{3 \times 3} q_{0}+\left[\boldsymbol{q}^{\times}\right]
\end{array}\right],
$$

$\boldsymbol{\omega}$ o vetor velocidade angular no sistema de coordenadas fixo do corpo, $\left[\boldsymbol{\omega}^{\times}\right]$e $\left[\boldsymbol{q}^{\times}\right]$as matrizes representativas do produto vetorial

$$
\mathscr{E}^{\times}=\left[\begin{array}{ccc}
0 & -\mathscr{E}_{3} & \mathscr{E}_{2} \\
\mathscr{E}_{3} & 0 & -\mathscr{E}_{1} \\
-\mathscr{E}_{2} & \mathscr{E}_{1} & 0
\end{array}\right]
$$

e $I_{3 \times 3}$ a matriz identidade no $\mathbb{R}^{3 \times 3}$. As velocidades angulares real $\boldsymbol{\omega}$ e estimada $\hat{\boldsymbol{\omega}}$ são modeladas da seguinte forma [38]

$$
\begin{aligned}
& \boldsymbol{\omega}=\boldsymbol{\omega}_{g}-\boldsymbol{b}_{g}-\boldsymbol{\eta}_{g}, \\
& \hat{\boldsymbol{\omega}}=\boldsymbol{\omega}_{g}-\hat{\boldsymbol{b}}_{g},
\end{aligned}
$$


sendo $\boldsymbol{\omega}_{g}$ a velocidade angular medida do giroscópio, $\boldsymbol{b}_{g}$ o bias do giroscópio e $\boldsymbol{\eta}_{g}$ o ruído branco gaussiano do giroscópio. Utilizando o processo de Gauss-Markov escalar para escrever o bias $\boldsymbol{b}_{g}$ tem-se

$$
\begin{aligned}
& \dot{\boldsymbol{b}}_{g}=-\frac{1}{\tau} \boldsymbol{b}_{g}+\boldsymbol{\eta}_{b g}, \\
& \dot{\hat{\boldsymbol{b}}}_{g}=0,
\end{aligned}
$$

sendo $\boldsymbol{\eta}_{b g}$ o ruído branco do bias do giroscópio e $\tau$ o tempo de correlação desse processo. Substituindo (3.3) em (3.1), obtém-se

$$
\begin{aligned}
\dot{q} & =\frac{1}{2} \Xi(q)\left(\boldsymbol{\omega}_{g}-\boldsymbol{b}_{g}\right)-\frac{1}{2} \Xi(q) \boldsymbol{\eta}_{g} \\
& =\frac{1}{2} \Omega\left(\boldsymbol{\omega}_{g}\right) q-\frac{1}{2} \Xi(q) \boldsymbol{b}_{g}-\frac{1}{2} \Xi(q) \boldsymbol{\eta}_{g} .
\end{aligned}
$$

A equação (3.7) não garante a norma unitária do quatérnio. Então será usada a equação

$$
q\left(t_{k}\right)=\left(\cos (\| \mathbf{w} \mid) I+\frac{\sin (\|\mathbf{w}\|)}{\|\mathbf{w}\|} W\right) q\left(t_{k-1}\right) .
$$

na discretização de (3.7) e em seguida, normaliza-se o quatérnio para manter a norma unitária. O desenvolvimento desta equação se encontra no apêndice A. Combinando as equações (3.7) e (3.5), e definindo o estado como $\mathbf{x}_{1}=\left[\begin{array}{ll}q^{T} & \boldsymbol{b}_{g}^{T}\end{array}\right]^{T} \in \mathbb{R}^{7 \times 1}$, obtém-se

$$
\dot{\mathbf{x}}_{1}=F_{1} \mathbf{x}_{1}+G_{1} \boldsymbol{\eta}_{1}
$$

sendo

$$
\begin{aligned}
F_{1} & =\left[\begin{array}{cc}
\frac{1}{2} \Omega\left(\boldsymbol{\omega}_{g}\right) & -\frac{1}{2} \Xi(q) \\
0_{3 \times 4} & -\frac{1}{\tau} I_{3 \times 3}
\end{array}\right], \\
G_{1} & =\left[\begin{array}{cc}
-\frac{1}{2} \Xi(q) & 0_{4 \times 3} \\
0_{3 \times 3} & I_{3 \times 3}
\end{array}\right] \text { e } \boldsymbol{\eta}_{1}=\left[\begin{array}{c}
\boldsymbol{\eta}_{g} \\
\boldsymbol{\eta}_{b g}
\end{array}\right] .
\end{aligned}
$$

A equação de medida é dada por

$$
\mathbf{z}_{1}=\mathbf{h}_{1}\left(\mathbf{x}_{1}\right)+\mathbf{v}_{1}
$$

sendo $\mathbf{h}_{1}\left(\mathbf{x}_{1}\right)=\left[\begin{array}{ll}\boldsymbol{a}^{T} & \psi\end{array}\right]^{T} \in \mathbb{R}^{4 \times 1}, \mathbf{v}_{1}$ processo gaussiano de média zero, $\boldsymbol{a} \in \mathbb{R}^{3 \times 1}$ a medida da aceleração no eixo do corpo do veículo e $\psi$ o ângulo de proa magnético gerado pelos magnetômetros. O ângulo de proa magnético é obtido através dos magnetômetros em 3 eixos, 
$m=\left[\begin{array}{lll}m_{x} & m_{y} & m_{z}\end{array}\right]^{T}$, utilizando o seguinte algoritmo

$$
\psi=\left\{\begin{array}{cc}
\frac{\pi}{2}-\tan ^{-1}\left(\frac{m_{x}}{m_{y}}\right) & \text { se } m_{y}>0 \\
\pi+\frac{\pi}{2}-\tan ^{-1}\left(\frac{m_{x}}{m_{y}}\right) & \text { se } m_{y}<0 \\
\frac{\pi}{2} & \text { se } m_{y}=0 \text { e } m_{x}<0 \\
0 & \text { se } m_{y}=0 \text { e } m_{x}>0
\end{array}\right.
$$

A medida da aceleração é dada por

$$
\boldsymbol{a}=\mathbf{R}_{b}^{g}(q) \boldsymbol{g}=\left[\begin{array}{c}
2 g\left(q_{1} q_{3}-q_{0} q_{2}\right) \\
2 g\left(q_{2} q_{3}+q_{0} q_{1}\right) \\
g\left(q_{0}^{2}-q_{1}^{2}-q_{2}^{2}+q_{3}^{2}\right)
\end{array}\right],
$$

sendo $g=\left[\begin{array}{lll}0 & 0 & g\end{array}\right]^{T}$ e $g=-9.81 \mathrm{~m} / \mathrm{s}^{2}$. O ângulo de proa é dado por

$$
\psi=\tan ^{-1}\left(\frac{2\left(q_{1} q_{2}+q_{0} q_{3}\right)}{q_{0}^{2}+q_{1}^{2}-q_{2}^{2}-q_{3}^{2}}\right) .
$$

Linearizando (3.11) em torno de $\mathbf{x}_{k}$, tem-se

$$
\mathbf{z}_{1}=H_{1} \mathbf{x}_{1}+\mathbf{v}_{1}, \operatorname{com} H_{1}=\frac{\partial \mathbf{z}(t)}{\partial \mathbf{x}_{k}}
$$

\subsection{Sistema de Navegação Inercial}

Sabe-se que receptores GPS podem fornecer informações de posição e velocidade de plataformas móveis com precisão em tarefas de agrimensura (processos de medição e representação do terreno). Porém, navegação usando apenas GPS não é uma boa solução. O problema é que os sinais do GPS estão sujeitos à interferência, podem facilmente ser obstruídos, suas medidas são transmitidas em baixas frequências e sua precisão cai muito com a perda de satélites. Assim, é interessante integrar o sistema de navegação GPS com um outro tipo de navegação para melhorar sua autonomia. Desse ponto de vista o INS é o ideal.

O INS é baseado em medições de acelerações lineares e velocidades angulares obtidas através de sensores, acelerômetros e giroscópios, que estão em um veículo cujos sinais não são sensíveis a interferências ou obstruções e permitem uma alta taxa de amostragem. A posição usando o INS é obtida através de uma dupla integração das acelerações e os ângulos de rotação do corpo através de uma simples integração das velocidades angulares. Porém, sua desvantagem 
está no crescimento do erro do INS decorrente da integração numérica. Isso acontece devido a falta de compensação do erros dos sensores, giroscópios e acelerômetros.

Assim, combinando GPS e INS, o INS fornece um posicionamento tridimensional de alta precisão enquanto o GPS, quando estiver disponível, mantém os erros de navegação em uma certa faixa, já que os erros de medidas do GPS não são função do tempo.

\subsubsection{Modelo em Espaço de Estado}

Conforme mostrado na Seção 2.3.1, se as velocidades no sistema de coordenadas geográfico, $v_{N E D}=\left[v_{n}, v_{e}, v_{d}\right]^{T}$, são conhecidas e as posições geodésicas no sistema de coordenadas LLA (latitude, longitude e altitude), $\boldsymbol{p}=[\phi, \lambda, h]^{T}$, são desejadas, então o vetor de variação geodésica LLA é relacionado com a velocidade NED através da Equação (2.3), repetida a seguir:

$$
\left[\begin{array}{c}
\dot{\phi} \\
\dot{\lambda} \\
\dot{h}
\end{array}\right]=\left[\begin{array}{ccc}
\frac{1}{R_{M}+h} & 0 & 0 \\
0 & \frac{1}{\left(R_{N}+h\right) \cos \phi} & 0 \\
0 & 0 & -1
\end{array}\right]\left[\begin{array}{c}
v_{n} \\
v_{e} \\
v_{d}
\end{array}\right]
$$

sendo $R_{M}$ e $R_{N}$ definidos pelas Equações (2.4) e (2.5), respectivamente.

A equação da aceleração NED é

$$
\boldsymbol{a}_{n e d}=\left[\begin{array}{c}
a_{n} \\
a_{e} \\
a_{d}
\end{array}\right]=\mathbf{R}_{b}^{g}(q)^{T} \boldsymbol{a}
$$

sendo $\boldsymbol{a}$ as medidas dos acelerômetros no sistema de coordenadas do corpo rígido e $\mathbf{R}_{b}^{g}(q)$ a matriz de cossenos diretores em termo do quatérnio $q$ entre o sistema de coordenadas geográfico $g$ e do corpo rígido $b$.

A aceleração do corpo rígido $\boldsymbol{a}$ e a estimada $\hat{\boldsymbol{a}}$ são modeladas da seguinte forma

$$
\begin{aligned}
& \boldsymbol{a}=\boldsymbol{a}_{a}-\boldsymbol{b}_{a}-\boldsymbol{\eta}_{a} \\
& \hat{\boldsymbol{a}}=\boldsymbol{a}_{a}-\hat{\boldsymbol{b}}_{a}
\end{aligned}
$$

sendo $\boldsymbol{a}_{a}$ a aceleração medida do acelerômetro, $\boldsymbol{b}_{a}$ o bias do acelerômetro e $\boldsymbol{\eta}_{a}$ o ruído branco gaussiano. 
Utilizando o processo de Gauss-Markov para escrever o bias $\boldsymbol{b}_{a}$, tem-se

$$
\begin{aligned}
& \dot{\boldsymbol{b}}_{a}=-\frac{1}{\tau} \boldsymbol{b}_{a}+\boldsymbol{\eta}_{b a}, \\
& \dot{\hat{\boldsymbol{b}}}_{a}=0
\end{aligned}
$$

sendo $\eta_{b a}$ o ruído branco do bias do acelerômetro e $\tau$ o tempo de correlação desse processo.

A equação da variação da velocidade no sistema geográfico como mostrado em [39] é

$$
\left[\begin{array}{c}
\dot{v}_{n} \\
\dot{v}_{e} \\
\dot{v}_{d}
\end{array}\right]=\left[\begin{array}{c}
a_{n} \\
a_{e} \\
a_{d}
\end{array}\right]-\left[\begin{array}{l}
0 \\
0 \\
g
\end{array}\right]+\left[\begin{array}{c}
-\frac{v_{e}^{2} \operatorname{sen}(\phi)}{\left(R_{N}+h\right) \cos (\phi)}+\frac{v_{n} v_{d}}{R_{M}+h} \\
\frac{v_{e} v_{n} \operatorname{sen}(\phi)}{\left(R_{N}+h\right) \cos (\phi)}+\frac{v_{e} v_{d}}{\left(R_{N}+h\right)} \\
-\frac{v_{e}^{2}}{R_{N}+h}-\frac{v_{n}^{2}}{R_{M}+h}
\end{array}\right]
$$

sendo $g=-9.81 \mathrm{~m} / \mathrm{s}^{2}$ a aceleração da gravidade.

Substitiuindo (3.16) em (3.15) e em seguida em (3.20), tem-se

$$
\left[\begin{array}{c}
\dot{v}_{n} \\
\dot{v}_{e} \\
\dot{v}_{d}
\end{array}\right]=\mathbf{R}_{b}^{g}(q)^{T}\left[\boldsymbol{a}_{a}-\boldsymbol{b}_{a}-\boldsymbol{\eta}_{a}\right]-\left[\begin{array}{c}
0 \\
0 \\
g
\end{array}\right]+\left[\begin{array}{c}
-\frac{v_{e}^{2} \operatorname{sen}(\phi)}{\left(R_{N}+h\right) \cos (\phi)}+\frac{v_{n} v_{d}}{R_{M}+h} \\
\frac{v_{e} v_{n} \operatorname{sen}(\phi)}{\left(R_{N}+h\right) \cos (\phi)}+\frac{v_{e} v_{d}}{\left(R_{N}+h\right)} \\
-\frac{v_{e}^{2}}{R_{N}+h}-\frac{v_{n}^{2}}{R_{M}+h}
\end{array}\right]
$$

Define-se o estado do sistema dinâmico como $\mathbf{x}_{2}=\left[\begin{array}{lll}\boldsymbol{p}^{T} & \boldsymbol{v}_{N E D}^{T} & \boldsymbol{b}_{a}^{T}\end{array}\right]^{T} \in \mathbb{R}^{9 \times 1}, \boldsymbol{\eta}_{2}=$ $\left[\begin{array}{ll}\boldsymbol{\eta}_{a}^{T} & \boldsymbol{\eta}_{b a}^{T}\end{array}\right]^{T} \in \mathbb{R}^{6 \times 1}$ como o ruído branco gaussiano e a equação de medida observável do GPS como $\mathbf{z}_{2}=\mathbf{h}_{2}\left(\mathbf{x}_{2}\right)+\mathbf{v}_{2} \in \mathbb{R}^{6 \times 1}$, sendo $\mathbf{h}_{2}(\mathbf{x})=\left[\begin{array}{ll}\boldsymbol{p}^{T} & \boldsymbol{v}_{N E D}^{T}\end{array}\right]^{T}$ e $\mathbf{v}_{2}$ o processo gaussiano de média zero. Linearizando (2.3), (3.21) e (3.18) em torno de $\mathbf{x}_{2}$, tem-se a dinâmica linearizada

$$
\begin{array}{lr}
\dot{\mathbf{x}}_{2}= & F_{2} \mathbf{x}_{2}+G_{2} \boldsymbol{\eta}_{2}, \\
\mathbf{z}_{2}= & H_{2} \mathbf{x}_{2}+\mathbf{v}_{2},
\end{array}
$$


sendo

$$
\begin{aligned}
& F_{2}=\left[\begin{array}{ccc}
F_{11} & F_{12} & 0_{3 \times 3} \\
F_{21} & F_{22} & -\mathbf{R}_{b}^{g}(q)^{T} \\
0_{3 \times 3} & 0_{3 \times 3} & -\frac{1}{\tau} I_{3 \times 3}
\end{array}\right] \\
& F_{11}=\left[\begin{array}{ccc}
0 & 0 & -\frac{v_{n}}{\left(R_{M}+h\right)^{2}} \\
\frac{v_{e} \operatorname{sen}(\phi)}{\left(R_{N}+h\right)(\cos (\phi))^{2}} & 0 & -\frac{v_{e}}{\left(R_{N}+h\right)^{2} \cos (\phi)} \\
0 & 0 & 0
\end{array}\right] \text {, } \\
& F_{12}=\left[\begin{array}{ccc}
\left(R_{M}+h\right)^{-1} & 0 & 0 \\
0 & \left(\left(R_{N}+h\right) \cos (\phi)\right)^{-1} & 0 \\
0 & 0 & -1
\end{array}\right] \\
& F_{21}=\left[\begin{array}{ccc}
-\frac{v_{e}^{2}}{R_{N}+h}-\frac{v_{e}^{2}(\operatorname{sen}(\phi))^{2}}{\left(R_{N}+h\right)(\cos (\phi))^{2}} & 0 & \frac{v_{e}^{2} \operatorname{sen}(\phi)}{\left(R_{N}+h\right)^{2} \cos (\phi)}-\frac{v_{n} v_{d}}{\left(R_{M}+h\right)^{2}} \\
\frac{v_{e} v_{n}}{R_{N}+h}+\frac{v_{e} v_{n}(\operatorname{sen}(\phi))^{2}}{\left(R_{N}+h\right)(\cos (\phi))^{2}} & 0 & -\frac{v_{e} v_{n} \operatorname{sen}(\phi)}{\left(R_{N}+h\right)^{2} \cos (\phi)}-\frac{v_{e} v_{d}}{\left(R_{N}+h\right)^{2}} \\
0 & 0 & \frac{v_{e}^{2}}{\left(R_{N}+h\right)^{2}}+\frac{v_{n}^{2}}{\left(R_{M}+h\right)^{2}}
\end{array}\right] \mathrm{e} \\
& F_{22}=\left[\begin{array}{ccc}
\frac{v_{d}}{R_{M}+h} & -2 \frac{v_{e} \operatorname{sen}(\phi)}{\left(R_{N}+h\right) \cos (\phi)} & \frac{v_{n}}{R_{M}+h} \\
\frac{v_{e} \operatorname{sen}(\phi)}{\left(R_{N}+h\right) \cos (\phi)} & \frac{v_{n} \operatorname{sen}(\phi)}{\left(R_{N}+h\right) \cos (\phi)}+\frac{v_{d}}{R_{N}+h} & \frac{v_{e}}{R_{N}+h} \\
-2 \frac{v_{n}}{R_{M}+h} & -2 \frac{v_{e}}{R_{N}+h} & 0
\end{array}\right] \text {, } \\
& G_{2}=\left[\begin{array}{cc}
0_{3 \times 3} & 0_{3 \times 3} \\
-\mathbf{R}_{b}^{g}(q)^{T} & 0_{3 \times 3} \\
0_{3 \times 3} & I_{3 \times 3}
\end{array}\right], \quad H_{2}=\left[\begin{array}{ll}
I_{6 \times 6} & 0_{6 \times 3}
\end{array}\right] \text {. }
\end{aligned}
$$

\subsection{Estimativa Robusta BDU}

Além dos ruídos de estado e de medida provenientes de sistemas dinâmicos, há também casos em que o modelo utilizado para representar o sistema não é exato o suficiente. Estas incertezas violam a premissa central da formulação do filtro de Kalman a qual assume que os parâmetros fundamentais do modelo $\{F, G, H\}$ são exatos. Quando esta suposição é violada, o desempenho do filtro pode deteriorar apreciavelmente.

Uma maneira de modelar essas incertezas é considerar os parâmetros $\{F, G\}$ como valores nominais e assumir que os valores reais variam em um certo conjunto cujos valores centrais são formados pelos parâmetros nominais. Assim, conforme mostrado em [34], considere o sistema 
de incertezas da forma

$$
\begin{aligned}
\mathbf{x}_{i+1} & =\left(F_{i}+\delta F_{i}\right) \mathbf{x}_{i}+\left(G_{i}+\delta G_{i}\right) \mathbf{w}_{i}, \quad i \geq 0, \\
\mathbf{z}_{i} & =H_{i} \mathbf{x}_{i}+\mathbf{v}_{i},
\end{aligned}
$$

em que $\left\{\mathbf{x}_{0}, \mathbf{w}_{i}, \mathbf{v}_{i}\right\}$ são variáveis aleatórias de média zero com variâncias

$$
E\left(\left[\begin{array}{c}
\mathbf{x}_{0} \\
\mathbf{w}_{i} \\
\mathbf{v}_{i}
\end{array}\right]\left[\begin{array}{c}
\mathbf{x}_{0} \\
\mathbf{w}_{j} \\
\mathbf{v}_{j}
\end{array}\right]^{T}\right)=\left[\begin{array}{ccc}
\Pi_{0} & 0 & 0 \\
0 & Q_{i} \delta_{i j} & 0 \\
0 & 0 & R_{i} \delta_{i j}
\end{array}\right]
$$

satisfazendo

$$
\Pi_{0}>0, \quad R_{i}>0, \quad Q_{i}>0
$$

Aqui, $\delta_{i j}$ é a função delta de Kronecker que é igual à unidade quando $i=j$ e zero, caso contrário. As pertubações em $\left\{F_{i}, G_{i}\right\}$ são modeladas como

$$
\left[\begin{array}{lll}
\delta & F_{i} & \delta G_{i}
\end{array}\right]=M_{i} \Delta_{i}\left[\begin{array}{ll}
E_{f, i} & E_{g, i}
\end{array}\right]
$$

para matrizes conhecidas $\left\{M_{i}, E_{f, i}, E_{g, i}\right\}$ e para uma contração arbitrária $\Delta_{i},\left\|\Delta_{i}\right\| \leq 1$. Observe que, de maneira geral, permite-se que as quantidades $\left\{M_{i}, E_{f, i}, E_{g, i}\right\}$ variem com o tempo. Para o caso onde haja incertezas somente em $F_{i}$, toma-se $E_{g, i}=0$. Da mesma maneira, para incertezas somente em $G_{i}$, toma-se $E_{f, i}=0$. Finalmente, para o caso de modelos exatos, toma-se $M_{i}=0$, $E_{f, i}=0$ e $E_{g, i}=0$.

O modelo (3.27) permite ao projetista restringir as fontes de distorção selecionando as entradas $\left\{E_{f, i} E_{g, i}\right\}$ apropriadamente [34].

Este filtro robusto procura minimizar o erro de estimativa para o pior caso possível criado pela faixa de incertezas $\delta F_{i}$ e $\delta G_{i}$. As estimativas $\left\{\hat{\mathbf{x}}_{i+1 \mid i}, \hat{\mathbf{x}}_{i+1 \mid i+1}\right\}$, no algoritmo que segue, podem ser obtidas por iterações recursivas entre os passos 2 e 3 mostrados abaixo. Para o caso quando $\hat{\lambda_{i}}=0$, os passos 2 e 3 são reduzidos para o tempo padrão e para as equações de atualização de medida do filtro de Kalman nominal. O algoritmo do filtro robusto, denominado BDU (Bounded Data Uncertainties) é dado abaixo:

Modelo de Incertezas:

Eqs. (3.23)-(3.27). $\Pi_{0}>0, R_{i}>0, Q_{i}>0$ são matrizes de ponderações.

Condições Iniciais:

$$
\hat{\mathbf{x}}_{0 \mid 0}=P_{0 \mid 0} H_{0}^{T} R_{0}^{-1} z_{0}
$$




$$
P_{0 \mid 0}=\left(\Pi_{0}^{-1}+H_{0}^{T} R_{0}^{-1} H_{0}\right)^{-1}
$$

\section{Passo 1:}

Se $H_{i+1} M_{i}=0$, então configure $\hat{\lambda}_{i}=0$ (filtro não robusto). Caso contrário, selecione $\alpha_{f i}>0$ e configure:

$$
\hat{\lambda}_{i}=\left(1+\alpha_{f i}\right) \cdot\left\|M_{i}^{T} H_{i+1}^{T} R_{i+1}^{-1} H_{i+1} M_{i}\right\|
$$

\section{Passo 2:}

Substitua $\left\{Q_{i}, R_{i}, P_{i \mid i}, G_{i}, F_{i}\right\}$ por:

$$
\begin{aligned}
\hat{Q}_{i}^{-1} & =Q_{i}^{-1}+\hat{\lambda}_{i} E_{g, i}^{T}\left[I+\hat{\lambda}_{i} E_{f, i} P_{i \mid i} E_{f, i}^{T}\right]^{-1} E_{g, i} \\
\hat{R}_{i+1} & =R_{i}-\hat{\lambda}_{i}^{-1} H_{i+1} M_{i} M_{i}^{T} H_{i+1}^{T} \\
\hat{P}_{i \mid i} & =P_{i \mid i}-P_{i \mid i} E_{f, i}^{T}\left[\hat{\lambda}_{i}^{-1} I+E_{f, i} P_{i \mid i} E_{f, i}^{T}\right]^{-1} E_{f, i} P_{i \mid i} \\
\hat{G}_{i} & =G_{i}-\hat{\lambda}_{i} F_{i} \hat{P}_{i \mid i} E_{f, i}^{T} E_{g, i} \\
\hat{F}_{i} & =\left(F_{i}-\hat{\lambda}_{i} \hat{G}_{i} \hat{Q}_{i} E_{g, i}^{T} E_{f, i}\right)\left(I-\hat{\lambda}_{i} \hat{P}_{i \mid i} E_{f, i}^{T} E_{f, i}\right)
\end{aligned}
$$

\section{Passo 3:}

Atualize $\left\{\hat{\mathbf{x}}_{i \mid i}, P_{i \mid i}\right\}$ como segue:

$$
\begin{aligned}
& \hat{\mathbf{x}}_{i+1 \mid i}=\hat{F}_{i} \hat{\mathbf{x}}_{i \mid i} \\
& \hat{\mathbf{x}}_{i+1 \mid i+1}=\hat{\mathbf{x}}_{i+1 \mid i}+P_{i+1 \mid i+1} H_{i+1}^{T} \hat{R}_{i+1}^{-1} e_{i+1} \\
& e_{i+1}=z_{i+1}-H_{i+1} \hat{\mathbf{x}}_{i+1 \mid i} \\
& P_{i+1 \mid i}=F_{i} \hat{P}_{i \mid i} F_{i}^{T}+\hat{G}_{i} \hat{Q}_{i} \hat{G}_{i}^{T} \\
& P_{i+1 \mid i+1}=P_{i+1 \mid i}-P_{i+1 \mid i} H_{i+1}^{T} R_{e, i+1}^{-1} H_{i+1} P_{i+1 \mid i} \\
& R_{e, i+1}=\hat{R}_{i+1}+H_{i+1} P_{i+1 \mid i} H_{i+1}^{T}
\end{aligned}
$$

\subsection{Estimativa Ótima Robusta}

Outra estimativa robusta proposta por [35] foi considerada. Uma vantagem desta abordagem em relação àquela proposta por [34] é a forma como as estimativas recursivas robustas, com as respectivas equações recursivas de Riccati, foram expressas na forma de blocos matriciais, possuindo estrutura simples e simétrica. A outra vantagem é que é mais fácil ajustar este 
filtro, basta considerarmos valores do parâmetro $\mu$ tendendo ao infinito para obtermos o ótimo robusto.

Considere o seguinte sistema dinâmico singular sujeito a incertezas paramétricas:

$$
\begin{aligned}
\left(E_{i+1}+\delta E_{i+1}\right) x_{i+1} & =\left(F_{i}+\delta F_{i}\right) x_{i}+\tilde{\mathrm{v}}_{i} \\
z_{i+1} & =\left(H_{i+1}+\delta H_{i+1}\right) x_{i+1}+\left(J_{i}+\delta J_{i}\right) x_{i}+\tilde{\mathrm{v}}_{i}, i \geq 0
\end{aligned}
$$

sendo $x_{i} \in \mathbb{R}^{n}$ a variável que descreve o comportamento interno do sistema; $z_{i} \in \mathbb{R}^{p}$ o sinal observado, $\tilde{v}_{i}$ o erro de ajuste do modelo definido como:

$$
\tilde{v}_{i}:=\left[\begin{array}{c}
\tilde{w}_{i} \\
\tilde{v}_{i+1}
\end{array}\right]:=\left[\begin{array}{c}
\left(G_{w, i}+\delta G_{w, i}\right) w_{i}+\left(G_{v, i+1}+\delta G_{v, i+1}\right) v_{i+1} \\
\left(K_{w, i}+\delta K_{w, i}\right) w_{i}+\left(K_{v, i+1}+\delta K_{v, i+1}\right) v_{i+1}
\end{array}\right] .
$$

As matrizes $E_{i+1}, F_{i}, G_{w, i}, G_{v, i+1}, H_{i+1}, J_{i}, K_{w, i}$ e $K_{v, i+1}$ são assumidas conhecidas, de dimensões apropriadas, quadradas ou retangulares; $\delta E_{i+1}, \delta F_{i}, \delta G_{w, i}, \delta G_{v, i+1}, \delta H_{i+1}, \delta J_{i}$, $\delta K_{w, i}$ e $\delta K_{v, i+1}$ são perturbações nos parâmetros do sistema nominal, variantes no tempo.

As incertezas paramétricas de (3.29) são modeladas por

$$
\left[\begin{array}{lll}
\delta F_{i} & \delta G_{i} & \delta E_{i+1} \\
\delta J_{i} & \delta K_{i} & \delta H_{i+1}
\end{array}\right]:=\left[\begin{array}{cc}
M_{1, i} & 0 \\
0 & M_{2, i}
\end{array}\right]\left[\begin{array}{cc}
\Delta_{1} & 0 \\
0 & \Delta_{2}
\end{array}\right]\left[\begin{array}{ccc}
N_{F_{i}} & N_{G_{i}} & N_{E_{i+1}} \\
N_{J_{i}} & N_{K_{i}} & N_{H_{i+1}}
\end{array}\right]
$$

sendo que $N_{G_{i}}:=\left[N_{G_{w, i}} N_{G_{v, i+1}}\right]$ e $N_{K_{i}}:=\left[N_{K_{w, i}} N_{K_{v, i+1}}\right]$.

O problema de ajuste ótimo para estimativas filtradas do sistema (3.29) é definido da seguinte maneira. Assuma que no passo $i$ tem-se a estimativa a priori para o estado $x_{i}$ e denote a estimativa inicial por $\hat{x}_{i \mid i}$. Além disso, suponha que há uma matriz de ponderação definida positiva $P_{i \mid i}$ para o erro de estimativa $\left(x_{i}-\hat{x}_{i \mid i}\right)$. Para atualizar a estimativa de $\hat{x}_{i \mid i}$ para $\hat{x}_{i+1 \mid i+1}$, o seguinte funcional associado a (3.29) é proposto

$$
\begin{aligned}
& \left.\mathscr{J}_{i}:=\left[\begin{array}{c}
x_{i}-\hat{x}_{i \mid i} \\
v_{i} \\
x_{i+1}
\end{array}\right]^{T}\left[\begin{array}{cc}
P_{i \mid i} & 0 \\
0 & \mathscr{R}_{i}
\end{array}\right]^{-1} \quad 0\right]\left[\begin{array}{c}
x_{i}-\hat{x}_{i \mid i} \\
v_{i} \\
x_{i+1}
\end{array}\right]+ \\
& \left.\left(\left[\begin{array}{c}
-F_{i} \hat{x}_{i \mid i} \\
z_{i+1}
\end{array}\right]+\left[\begin{array}{l}
-\delta F_{i} \hat{x}_{i \mid i} \\
-\delta J_{i} \hat{x}_{i \mid i}
\end{array}\right]\right)+\left(\left[\begin{array}{ccc}
F_{i} & G_{i} & -E_{i+1} \\
J_{i} & K_{i} & H_{i+1}
\end{array}\right]+\left[\begin{array}{ccc}
\delta F_{i} & \delta G_{i} & -\delta E_{i+1} \\
\delta J_{i} & \delta K_{i} & \delta H_{i+1}
\end{array}\right]\right)\left[\begin{array}{c}
x_{i}-\hat{x}_{i \mid i} \\
v_{i} \\
x_{i+1}
\end{array}\right]\right)^{T} \\
& \times \Xi_{i}^{-1}\left(\left[\begin{array}{l}
\bullet \\
{[}
\end{array}\right]\right)
\end{aligned}
$$


com

$$
\begin{aligned}
v_{i} & :=\left[\begin{array}{c}
w_{i} \\
v_{i+1}
\end{array}\right], G_{i}:=\left[G_{w, i} G_{v, i+1}\right], K_{i}:=\left[K_{w, i} K_{v, i+1}\right], \\
\mathscr{R}_{i} & :=\left[\begin{array}{cc}
Q_{i} & S_{i} \\
S_{i}^{T} & R_{i+1}
\end{array}\right] \text { e } \quad \Xi_{i}:=\mu_{i}^{-1}\left[\begin{array}{cc}
\theta_{11} & \theta_{12} \\
\theta_{21} & \theta_{22}
\end{array}\right]^{-1} .
\end{aligned}
$$

O problema de filtragem robusta é encontrar $\hat{x}_{i+1 \mid i+1}$ que minimize $\mathscr{J}_{i}$, considerando o pior caso das perturbações, ou seja,

$$
\min _{x_{i}, x_{i+1}} \max _{\delta_{i}} \mathscr{J}_{i}
$$

sendo $\delta_{i}:=\left\{\delta E_{i+1}, \delta F_{i}, \delta G_{i}, \delta H_{i+1}, \delta J_{i}, \delta K_{i}\right\}$.

O resultado apresentado no Teorema 3.4.1, cuja prova pode ser verificada em [35], fornece expressões para as estimativas robustas ótimas na forma filtrada com a respectiva equação recursiva de Riccati. 
Teorema 3.4.1 Considere o sistema dinâmico singular (3.29) e o problema de otimização (3.34), sendo as incertezas paramétricas dadas por (3.31). Suponha que

$$
\left[E_{i+1}^{T} H_{i+1}^{T} N_{E_{i+1}}^{T} N_{H_{i+1}}^{T}\right] e\left[\begin{array}{ccc}
F_{i} & G_{i} & E_{i+1} \\
J_{i} & K_{i} & H_{i+1} \\
N_{F_{i}} & N_{G_{i}} & N_{E_{i+1}} \\
N_{J_{i}} & N_{K_{i}} & N_{H_{i+1}}
\end{array}\right]
$$

tenham posto linha pleno para todo $i$. Assim, tem-se que as estimativas robustas filtradas $\hat{x}_{i+1 \mid i+1}$ e sua correspondente equação recursiva de Riccati são dadas por

$$
\left[\hat{x}_{i+1 \mid i+1} P_{i+1 \mid i+1}\right]=\left[\begin{array}{c}
\mathbf{0} \\
\mathbf{0} \\
\mathbf{0} \\
\mathbf{0} \\
\mathbf{0} \\
\mathbf{0} \\
\mathbf{I}
\end{array}\right]^{T}\left[\begin{array}{ccccccc}
-\mathscr{Q}_{i} & \mathbf{0} & \mathbf{0} & \mathbf{I} & \mathbf{0} & \mathbf{0} & \mathbf{0} \\
\mathbf{0} & -\hat{\mathscr{W}}_{i} & \mathbf{0} & \mathbf{0} & \mathbf{I} & \mathbf{0} & \mathbf{0} \\
\mathbf{0} & \mathbf{0} & -\hat{\lambda}_{i} \mathbf{I} & \mathbf{0} & \mathbf{0} & \mathbf{I} & \mathbf{0} \\
\mathbf{I} & \mathbf{0} & \mathbf{0} & \mathbf{0} & \mathbf{0} & \mathbf{0} & \mathbf{I} \\
\mathbf{0} & \mathbf{I} & \mathbf{0} & \mathbf{0} & \mathbf{0} & \mathbf{0} & \mathscr{A}_{i} \\
\mathbf{0} & \mathbf{0} & \mathbf{I} & \mathbf{0} & \mathbf{0} & \mathbf{0} & \mathscr{N}_{\mathscr{A}_{i}} \\
\mathbf{0} & \mathbf{0} & \mathbf{0} & \mathbf{I} & \mathscr{A}_{i}^{T} & \mathscr{N}_{\mathscr{A}_{i}}^{T} & \mathbf{0}
\end{array}\right]^{-1}\left[\begin{array}{cc}
\mathbf{0} & \mathbf{0} \\
\mathbf{0} & \mathbf{0} \\
\mathbf{0} & \mathbf{0} \\
\hat{\mathbb{X}}_{i} & \mathbf{0} \\
\mathbb{Z}_{i+1} & \mathbf{0} \\
\mathbf{0} & \mathbf{0} \\
\mathbf{0} & \mathbf{I}
\end{array}\right]
$$

sendo que

$$
\begin{aligned}
\mathscr{Q}_{i} & :=\left[\begin{array}{ccc}
P_{i \mid i}^{-1} & \mathbf{0} & \mathbf{0} \\
\mathbf{0} & \mathscr{R}_{i}^{-1} & \mathbf{0} \\
\mathbf{0} & \mathbf{0} & \mathbf{0}
\end{array}\right], \mathscr{A}_{i}:=\left[\begin{array}{ccc}
F_{i} & G_{i} & -E_{i+1} \\
J_{i} & K_{i} & H_{i+1}
\end{array}\right] \\
\mathscr{N}_{\mathscr{A}_{i}} & :=\left[\begin{array}{ccc}
N_{F_{i}} & N_{G_{i}} & -N_{E_{i+1}} \\
N_{J_{i}} & N_{K_{i}} & -N_{H_{i+1}}
\end{array}\right], \mathbb{Z}_{i+1}:=\left[\begin{array}{c}
\mathbf{0} \\
z_{i+1}
\end{array}\right], \hat{\mathbb{X}}_{i}:=\left[\begin{array}{c}
\hat{x}_{i \mid i} \\
\mathbf{0} \\
\mathbf{0}
\end{array}\right] .
\end{aligned}
$$

Além disso, tem-se que

$$
\hat{\mathscr{W}}_{i}=\left(\Xi_{i}-\hat{\lambda}_{i}^{-1} M_{i} M_{i}^{T}\right)^{-1}
$$

sendo $\Xi:=\mu_{i}^{-1}\left[\begin{array}{ll}\theta_{11} & \theta_{12} \\ \theta_{21} & \theta_{22}\end{array}\right]^{-1}$ e $M_{i}:=\left[\begin{array}{cc}M_{1, i} & 0 \\ 0 & M_{2, i}\end{array}\right]$ para $\mu_{i}>0$ fixado. O ótimo robusto será obtido para $\mu \rightarrow \infty$.

O parâmetro $\hat{\lambda}_{i}$ deve satisfazer a seguinte desigualdade $\hat{\lambda}_{i} \geq\left\|M_{i}^{T} \Xi^{-1} M_{i}\right\|$ e minimizar uma função $G(\lambda)$. 


\section{Modelo Dinâmico do Helicóptero}

Este capítulo apresenta o modelo em espaço de estado para o helicóptero utilizado no desenvolvimento das leis de controle deste trabalho. Primeiro serão descritas as equações dinâmicas do helicóptero e depois será apresentado um modelo linearizado para o helicóptero no modo hover [18].

\subsection{Visão Geral}

Uma aeronave pode ser considerada como um corpo rígido de seis graus de liberdade, sendo três graus de liberdade rotacionais em torno dos eixos $x, y$ e $z$ e três graus de liberdade translacionais ao longo dos mesmos eixos. O modelo em questão consiste de um total de quatro entradas de controle e doze variáveis de estado. As entradas e os estados deste modelo são: (entradas)

- $u_{\text {lat }}$ : comando cíclico lateral;

- $u_{l o n}$ : comando cíclico longitudinal;

- $u_{c o l}$ : comando coletivo do rotor principal;

- $u_{\text {ped }}$ : comando coletivo da cauda (guinada).

(estados)

- $u$ : velocidade longitudinal ao longo do eixo $x$;

- $v$ : velocidade lateral ao longo do eixo $y$;

- $w$ : velocidade vertical de subida/descida ao longo do eixo $z$;

- $p$ : velocidade angular em torno do eixo $x$; 
- $q$ : velocidade angular em torno do eixo $y$;

- $r$ : velocidade angular em torno do eixo $z$;

- $\phi$ : ângulo de rolagem, rotação em torno do eixo $x$;

- $\theta$ : ângulo de arfagem, rotação em torno do eixo $y$;

- $\psi$ : ângulo de guinada, rotação em torno do eixo $z$;

- $x$ : eixo $x$, apontando para o Norte verdadeiro;

- $y$ : eixo $y$, apontando para o Leste;

- $z$ : eixo $z$, apontando para baixo.

Adicionalmente, introduz-se o vetor de distúrbios causados pelo vento $\mathrm{w}=\left[u_{w}, v_{w}, w_{w}\right]^{T}$.

Os comandos cíclicos lateral e longitudinal são assim chamados pois mudam o passo das pás do rotor ciclicamente. O resultado é a inclinação do disco do rotor em uma determinada direção, fazendo com que o helicóptero se mova nessa direção. Se houver comando empurrando o cíclico longitudinal para a frente, o disco de rotor inclina para a frente e o rotor produz um impulso para a frente. Se houver comando empurrando o cíclico para a direita, o disco do rotor inclina para a direita, produzindo impulso nesse sentido, fazendo com que o helicóptero paire lateralmente. O comando coletivo do rotor principal muda o ângulo de arfagem de todas as pás do mesmo coletivamente (ou seja, todos ao mesmo tempo) e independente de sua posição. Portanto, se uma entrada colectiva é gerada, todas as lâminas mudam de forma igual e o resultado é o helicóptero aumentando ou diminuindo em altitude. A função do comando coletivo de cauda é controlar a direção para a qual o nariz da aeronave aponta. A aplicação do comando em uma dada direção muda o passo das pás do rotor de cauda, aumentando ou reduzindo o empuxo produzido pelo rotor da cauda e fazendo com que o nariz guine na direção desejada. O comando de cauda muda mecanicamente a arfagem do rotor de cauda, alterando a quantidade de empuxo produzido.

A relação entrada/saída será descrita em um modelo de espaço de estado linearizado:

$$
\dot{\mathbf{x}}=A \mathbf{x}+B \mathbf{u},
$$

com os estados $\mathbf{x}$ e as entradas de controle $\mathbf{u}$ descritos acima. 


\subsection{Forças, Momentos e Equações Cinemáticas}

Para obter um modelo de uma aeronave, as equações de movimento necessitam ser desenvolvidas a partir de equações de força e momento que, juntamente com as equações cinéticas, resultam no modelo de espaço de estado. A Figura 4.1 exibe um diagrama de blocos ilustrando a complexidade do modelo matemático de um helicóptero.

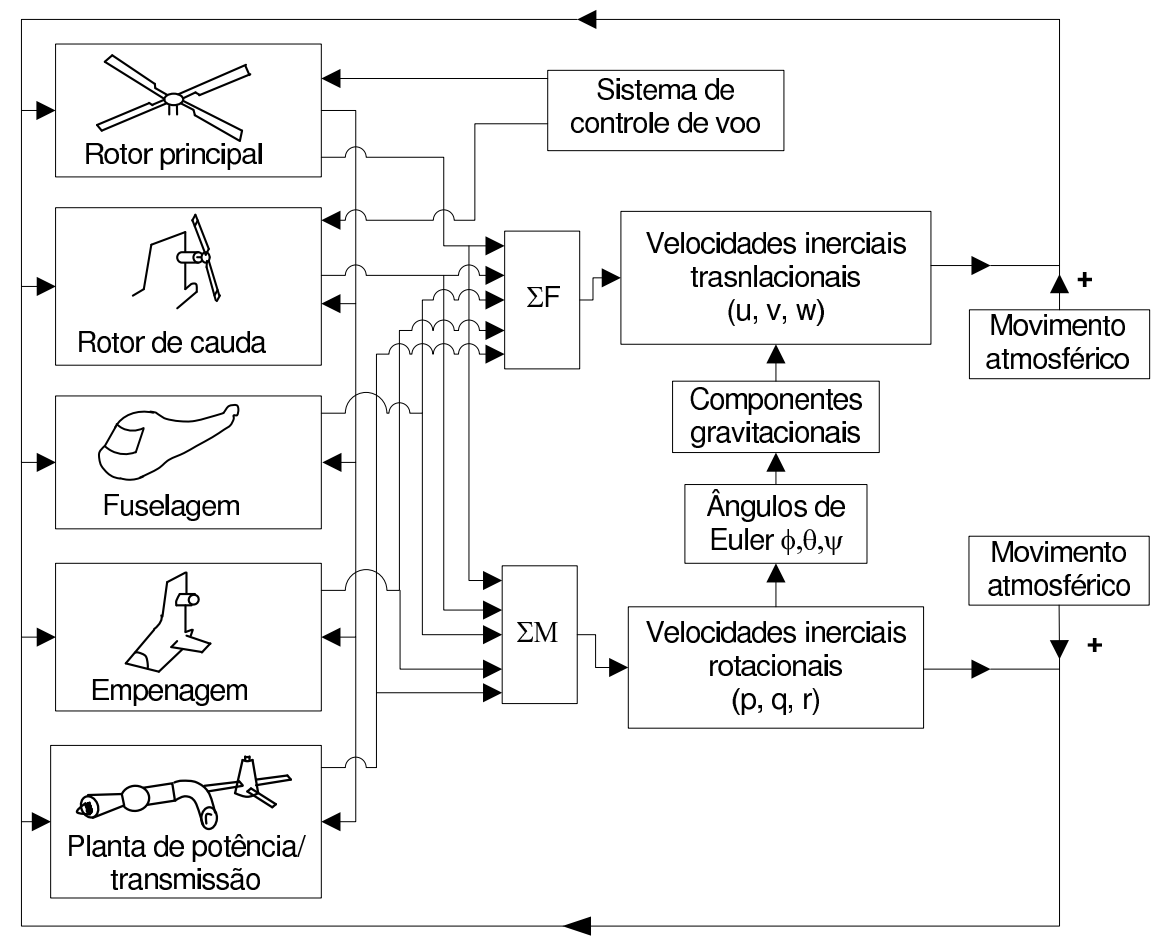

Figura 4.1: Modelo do helicóptero em diagrama de blocos.

O modelo do helicóptero pode ser descrito como um modelo genérico de um corpo rígido de 6 graus de liberdade com forças externas e momentos originados dos rotores principal e cauda, empenagem e fuselagem, conforme descrito pelas seguintes equações:

$$
\begin{aligned}
\dot{u} & =v r-w q-g \operatorname{sen} \theta+X / m \\
\dot{v} & =w p-u r+g \cos \theta \operatorname{sen} \phi+Y / m \\
\dot{w} & =u q-v p+g \cos \theta \cos \phi+Z / m \\
\dot{p} & =q r\left(I_{y y}-I_{z z}\right) / I_{x x}+L / I_{x x} \\
\dot{q} & =r p\left(I_{z z}-I_{x x}\right) / I_{y y}+M / I_{y y} \\
\dot{r} & =p q\left(I_{x x}-I_{y y}\right) / I_{z z}+N / I_{z z} \\
\left(\begin{array}{lll}
\dot{\phi} & \dot{\theta} & \dot{\psi}
\end{array}\right)^{T} & =\Psi(\phi, \theta, \Psi)\left(\begin{array}{lll}
p & q & r
\end{array}\right)^{T} \\
\left(\begin{array}{ccc}
\dot{x} & \dot{y} & \dot{z}
\end{array}\right)^{T} & =\mathbf{R}(\phi, \theta, \psi)\left(\begin{array}{lll}
u & v & w
\end{array}\right)^{T}
\end{aligned}
$$


sendo $I_{x x}, I_{y y}, I_{z z}$ são momentos de inércia (o produto de inércia para este caso é desprezado), $\Psi(\phi, \theta, \psi)$ descreve uma transformação na velocidade angular e $\mathbf{R}(\phi, \theta, \psi) \in S O(3)$ é uma matriz de transformação da velocidade linear. $X, Y, Z, L, M, N$ são forças e momentos induzidos pelo rotor principal, fuselagem, rotor de cauda, asa vertical e asa horizontal, sendo exibidos a seguir:

$$
\begin{aligned}
& X=X_{m r}+X_{f u s} \\
& Y=Y_{m r}+Y_{f u s}+Y_{t r}+Y_{v f} \\
& Z=Z_{m r}+Z_{f u s}+Z_{h t} \\
& L=L_{m r}+L_{t r}+L_{v f} \\
& M=M_{m r}+M_{h t} \\
& N=-Q_{e}+N_{v f}+N_{t r}
\end{aligned}
$$

O conjunto de forças e momentos atuando no helicóptero são organizados por componente: ()$_{m r}$ para rotor principal; ()$_{t r}$ para rotor de cauda; ()$_{f u s}$ para fuselagem; ()$_{v f}$ para asa vertical e ()$_{h t}$ para asa horizontal. As equações para cada componente estão disponíveis em [9].

As equações dinâmicas genéricas de 6 graus de liberdade são também complementadas com as equações da dinâmica flapping do rotor principal lateral e logintudinal, as quais são aproximadas como estado permanente para o propósito de derivação da lei de controle

$$
\begin{aligned}
& b_{1}=-\tau_{f b} p+\frac{\partial b_{1}}{\partial \mu_{v}} \frac{v-v_{\omega}}{V_{t i p}}+K_{l a t} u_{l a t} \\
& a_{1}=-\tau_{f b} q+\frac{\partial a_{1}}{\partial \mu} \frac{u-u_{\omega}}{V_{t i p}}+\frac{\partial a_{1}}{\partial \mu_{z}} \frac{\omega-\omega_{\omega}}{V_{t i p}}+K_{l o n} u_{l o n}
\end{aligned}
$$

sendo $\frac{\partial a_{1}}{\partial \mu}=-\frac{\partial b_{1}}{\partial \mu_{v}}=2 K_{\mu}\left(4 / 3 u_{c o l}-\lambda_{0}\right)$. A aproximação do flapping torna-se parte da equação algébrica não-linear das forças induzidas do rotor principal e dos momentos nas equações de 6 graus de liberdade. Segundo [21], tal aproximação resulta de inúmeros modos ressonantes nas dinâmicas de rolagem e arfagem. Simulações mostram que oscilações excessivas podem surgir quando os parâmetros do modelo são pouco precisos.

\subsection{Modelo Yamaha $R-M A X$ Adaptado}

Para simular os filtros e o sistema de controle, será utilizada uma modificação do modelo linearizado do helicóptero Yamaha $R-M A X$ em modo de voo pairado proposto em [2]. Dois modelos linearizados foram obtidos, em modo de voo de cruzeiro e em modo pairado, utilizando $\mathrm{CIFER}^{\circledR}$, um aplicativo baseado em um método de identificação de sistemas no domínio da 
frequência [40] que, simplificadamente, extrai a descrição matemática do veículo a partir de dados de teste. A Figura 4.2 exibe a aeronave em questão e a Figura 4.3 exibe um diagrama de blocos ilustrando as etapas do sistema CIFER $^{\circledR}$. Experimentos com os modelos linearizados foram utlizados em [18] e [41].

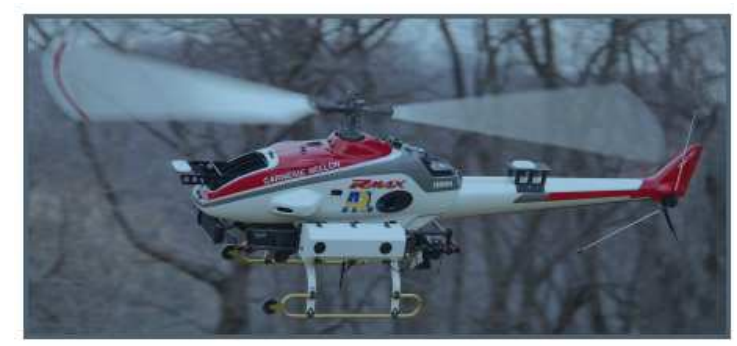

Figura 4.2: Yamaha R-MAX.

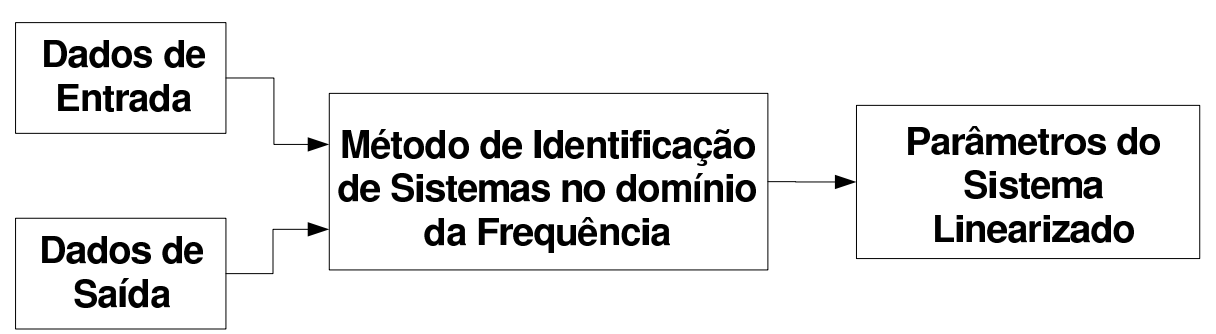

Figura 4.3: Diagrama de blocos ilustrando as etapas do aplicativo CIFER.

A Tabela 4.1 exibe as principais características físicas da aeronave.

Tabela 4.1: Características físicas do Yamaha R-MAX.

\begin{tabular}{|c|c|c|c|}
\hline Parâmetro & Descrição & Unidade & Valor \\
\hline $\mathrm{R}$ & Raio do rotor & $\mathrm{m}$ & 1,555 \\
\hline $\mathrm{M}$ & Massa & $\mathrm{kg}$ & 74 \\
\hline$I_{x x}$ & Inércia de rolagem & $\mathrm{kg} \cdot \mathrm{m}^{2}$ & 3,246 \\
\hline$I_{y y}$ & Inércia de arfagem & $\mathrm{kg} \cdot \mathrm{m}^{2}$ & 11,229 \\
\hline$\Omega$ & Velocidade do rotor & $\mathrm{rad} / \mathrm{s}$ & 90 \\
\hline$I_{\beta}$ & Inércia da pá & $\mathrm{kg} \cdot \mathrm{m}^{2}$ & 1,420 \\
\hline
\end{tabular}

A fim de se aplicar um controlador $\mathscr{H}_{\infty}$ não-linear, adiciona-se ao modelo dinâmico linear duas matrizes de rotação, deixando-o dependente dos estados $\phi, \theta$ e $\psi$. Desta forma, o modelo adotado possui 20 estados, 6 graus de liberdade e dinâmica acoplada dos rotores principal e cauda, empenagem e fuselagem. Tomando $\rho=\{\phi, \theta \psi\}$, o modelo é dado por:

$$
\dot{\mathbf{x}}=\mathbf{A}(\rho) \mathbf{x}+\mathbf{B}_{1} \mathbf{w}+\mathbf{B}_{2} \mathbf{u},
$$

onde $\mathbf{x}$ é o vetor de estado, $\mathbf{u}$ é a entrada de controle e $\mathbf{w}$ representa o distúrbio do vento. 
A Tabela 4.2 apresenta os elementos do vetor de estado $\mathbf{x}$, do vetor de entradas $\mathbf{u}$ e uma breve descrição sobre os mesmos.

Tabela 4.2: Elementos dos Vetores de Estado e de Entradas.

\begin{tabular}{|c|c|}
\hline Elemento & Descrição \\
\hline$u^{B}, \quad v^{B}, \quad w^{B}$ & Velocidades lineares do helicóptero no sistema de referência do corpo. \\
\hline$p, \quad q, \quad r$ & Velocidades angulares do helicóptero. \\
\hline$\phi, \quad \theta, \quad \psi$ & Ângulos de rolagem, arfagem e guinada, respectivamente. \\
\hline$x^{B}, \quad y^{B}, \quad z^{B}$ & Posição do helicóptero no sistema de referência do corpo. \\
\hline$r_{f b}$ & Realimentação da taxa de guinada. \\
\hline$\beta_{l c}, \quad \beta_{l s}$ & Arfagem cíclica lateral e longitudinal das pás principais. \\
\hline$k_{l c}, \quad k_{l s}$ & Arfagem cíclica lateral e longitudinal das pás estabilizadoras. \\
\hline$\beta_{0}, \quad \dot{\beta_{0}}$ & Ângulo entre a referência e as pás principais, quando em vôo, e sua variação. \\
\hline $\mathrm{v}$ & Fluxo de ar. \\
\hline$u_{\text {lat }}$ & Comando lateral. \\
\hline$u_{\text {lon }}$ & Comando longitudinal. \\
\hline$u_{c o l}$ & Comando coletivo. \\
\hline$u_{\text {ped }}$ & Comando do pedal (guinada). \\
\hline
\end{tabular}

As matrizes $\mathbf{A}(\rho)$ e $\mathbf{B}_{2}$ possuem as seguintes estruturas:

$$
\begin{aligned}
\mathbf{A}(\rho)= & {\left[\begin{array}{ccccccc}
\mathbf{A}_{1} & \mathbf{A}_{3} & \mathbf{A}_{7} & \mathbf{0}_{3 \times 3} & \mathbf{A}_{8} & \mathbf{0}_{3 \times 2} & \mathbf{A}_{13} \\
\mathbf{A}_{2} & \mathbf{A}_{4} & \mathbf{0}_{3 \times 3} & \mathbf{0}_{3 \times 3} & \mathbf{A}_{9} & \mathbf{0}_{3 \times 2} & \mathbf{0}_{3 \times 3} \\
\mathbf{0}_{3 \times 3} & \Psi & \mathbf{0}_{3 \times 3} & \mathbf{0}_{3 \times 3} & \mathbf{0}_{3 \times 3} & \mathbf{0}_{3 \times 2} & \mathbf{0}_{3 \times 3} \\
\mathbf{R}_{b}^{g} & \mathbf{0}_{3 \times 3} & \mathbf{0}_{3 \times 3} & \mathbf{0}_{3 \times 3} & \mathbf{0}_{3 \times 3} & \mathbf{0}_{3 \times 2} & \mathbf{0}_{3 \times 3} \\
\mathbf{0}_{3 \times 3} & \mathbf{A}_{5} & \mathbf{0}_{3 \times 3} & \mathbf{0}_{3 \times 3} & \mathbf{A}_{10} & \mathbf{A}_{11} & \mathbf{0}_{3 \times 3} \\
\mathbf{0}_{2 \times 3} & \mathbf{A}_{6} & \mathbf{0}_{2 \times 3} & \mathbf{0}_{2 \times 3} & \mathbf{0}_{2 \times 3} & \mathbf{A}_{12} & \mathbf{0}_{2 \times 3} \\
\mathbf{0}_{3 \times 3} & \mathbf{0}_{3 \times 3} & \mathbf{0}_{3 \times 3} & \mathbf{0}_{3 \times 3} & \mathbf{0}_{3 \times 3} & \mathbf{0}_{3 \times 2} & \mathbf{A}_{14}
\end{array}\right] } \\
\mathbf{B}_{1}= & -\left[\begin{array}{llllllll}
\mathbf{A}_{3}^{T} & \mathbf{A}_{4}^{T} & \Psi^{T} & \mathbf{0}_{3 \times 3}^{T} & \mathbf{A}_{5}^{T} & \mathbf{A}_{6}^{T} & \mathbf{0}_{3 \times 3}^{T}
\end{array}\right]^{T} \\
\mathbf{B}_{2}= & {\left[\begin{array}{lll}
\mathbf{B}_{2_{1}}^{T} & \mathbf{0}_{7 \times 4}^{T} & \mathbf{B}_{22}^{T}
\end{array}\right]^{T} }
\end{aligned}
$$

As matrizes $\mathbf{A}_{i}$ e $\mathbf{B}_{2_{i}}$ são definidas como segue:

$$
\mathbf{A}_{1}=\left[\begin{array}{ccc}
X_{u} & X_{v} & X_{w} \\
Y_{u} & Y_{v} & Y_{w} \\
Z_{u} & Z_{v} & Z_{w}
\end{array}\right], \quad \mathbf{A}_{2}=\left[\begin{array}{ccc}
L_{u} & L_{v} & L_{w} \\
M_{u} & M_{v} & M_{w} \\
N_{u} & N_{v} & N_{w}
\end{array}\right]
$$




$$
\begin{aligned}
& \mathbf{A}_{3}=\left[\begin{array}{ccc}
0 & -W_{0} & \left(X_{r}-W_{0}\right) \\
W_{0} & 0 & \left(Y_{r}-U_{0}\right) \\
\left(Z_{p}-V_{0}\right) & \left(U_{0}+Z_{q}\right) & Z_{r}
\end{array}\right], \quad \mathbf{A}_{4}=\left[\begin{array}{ccc}
0 & 0 & L_{r} \\
0 & 0 & M_{r} \\
N_{p} & N_{q} & N_{r}
\end{array}\right] \\
& \mathbf{A}_{5}=\left[\begin{array}{ccc}
0 & 0 & -K_{r} \\
0 & 1 & 0 \\
1 & 0 & 0
\end{array}\right], \quad \mathbf{A}_{6}=\left[\begin{array}{lll}
0 & 1 & 0 \\
1 & 0 & 0
\end{array}\right] \\
& \mathbf{A}_{7}=\left[\begin{array}{ccc}
0 & -g \operatorname{sen} \theta & 0 \\
g \operatorname{sen} \phi \cos \theta & 0 & 0 \\
0 & g \cos \phi \cos \theta & 0
\end{array}\right], \quad \mathbf{A}_{8}=\left[\begin{array}{ccc}
0 & X_{\beta_{1 c}} & 0 \\
0 & 0 & Y_{\beta_{1 s}} \\
0 & 0 & 0
\end{array}\right], \\
& \mathbf{A}_{9}=\left[\begin{array}{ccc}
0 & 0 & L_{\beta_{1 s}} \\
0 & M_{\beta_{1 c}} & 0 \\
-N_{r_{f b}} & 0 & 0
\end{array}\right], \quad \mathbf{A}_{10}=\left[\begin{array}{ccc}
K_{r_{f b}} & 0 & 0 \\
0 & -\frac{1}{\tau_{f}} & \frac{M_{\beta_{\beta_{1 c}}}}{\tau_{f}} \\
0 & \frac{L_{\beta_{\beta_{1 c}}}}{\tau_{f}} & -\frac{1}{\tau_{f}}
\end{array}\right] \\
& \mathbf{A}_{11}=\left[\begin{array}{cc}
\frac{M_{f_{K_{1 s}}}}{\tau_{f}} & 0 \\
0 & \frac{L_{f_{k_{1 s}}}}{\tau_{f}}
\end{array}\right], \quad \mathbf{A}_{12}=\left[\begin{array}{cc}
-\frac{1}{\tau_{s}} & 0 \\
0 & -\frac{1}{\tau_{s}}
\end{array}\right] \\
& \mathbf{A}_{13}=\left[\begin{array}{ccc}
0 & 0 & 0 \\
0 & 0 & 0 \\
\frac{-\rho \pi R^{2}(\Omega R)^{2}}{m C_{0}} \frac{4 \bar{v}_{0}}{3 \Omega} & 0 & {\left[\frac{1.2727 \rho \pi^{2} \Omega R^{3}}{m C_{0}}\left(\overline{\mathrm{v}}_{0}+\frac{C_{L_{\alpha} \sigma}}{16}\right) C_{0}+\frac{4 \overline{\mathrm{v}}_{0}}{\Omega R}\right]}
\end{array}\right], \\
& \mathbf{A}_{14}=\left[\begin{array}{ccc}
-\frac{\Omega \gamma}{8} & -\Omega^{2} & -\frac{\Omega \gamma}{6 R} \\
0 & 1 & 0 \\
-v_{\beta_{0}} & 0 & -\frac{75 \pi \Omega}{32}\left(\bar{v}_{0}+\frac{C_{L_{\alpha}} \sigma}{16}\right) C_{0}
\end{array}\right]
\end{aligned}
$$$$
\mathbf{R}_{b}^{g}=\left[\begin{array}{ccc}
c \theta c \psi & -c \phi s \psi+s \phi s \theta c \psi & s \phi s \psi+c \phi s \theta c \psi \\
c \theta s \psi & c \phi c \psi+s \phi s \theta s \psi & -s \phi c \psi+c \phi s \theta s \psi \\
-s \theta & s \phi c \theta & c \phi c \theta
\end{array}\right], \quad \Psi=\left[\begin{array}{ccc}
1 & s \phi t \theta & c \phi t \theta \\
0 & c \phi & -s \phi \\
0 & s \phi / c \theta & c \phi / c \theta
\end{array}\right]
$$ 


$$
\begin{aligned}
& \mathbf{B}_{2_{1}}=\left[\begin{array}{cccc}
0 & 0 & X_{c o l} & X_{r} \\
0 & 0 & Y_{u_{c o l}} & Y_{u_{p e d}} \\
Z_{u_{l a t}} & Z_{u_{l o n}} & -\frac{0.4242 \rho \pi^{2} \Omega^{2} R^{4}}{m C_{0}}\left(\frac{C_{L_{\alpha}} \sigma}{8}\right) C_{0} K_{\theta_{0}} & Z_{u_{p e d}} \\
0 & 0 & L_{u_{c o l}} & L_{u_{p e d}} \\
0 & 0 & M_{u_{c o l}} & M_{u_{p e d}} \\
N_{u_{l a t}} & N_{u_{l o n}} & N_{u_{c o l}} & N_{u_{p e d}}
\end{array}\right], \\
& \mathbf{B}_{2_{2}}=\left[\begin{array}{cccc}
\frac{M_{f_{u_{l a t}}}}{\tau_{f}} & \frac{M_{f_{u_{l o n}}}}{\tau_{f}} & 0 & 0 \\
\frac{L_{f_{u_{l a t}}}}{\tau_{f}} & \frac{L_{f_{u_{l o n}}}}{\tau_{f}} & 0 & 0 \\
0 & \frac{M_{s_{u_{l o n}}}}{\tau_{s}} & 0 & 0 \\
\frac{L_{s_{u_{l a t}}}}{\tau_{s}} & 0 & 0 & 0 \\
0 & 0 & \frac{\Omega^{2} \gamma}{8} K_{\theta_{0}} & 0 \\
0 & 0 & 0 & 0 \\
0 & 0 & \frac{25 \pi \Omega^{2} R}{32}\left(\frac{C_{L_{\alpha}} \sigma}{8}\right) C_{0} K_{\theta_{0}} & 0
\end{array}\right] .
\end{aligned}
$$




\section{$5 \quad$ Sistema de Controle}

Este capítulo apresenta a estratégia de controle considerada para definir a posição e a atitude do R-MAX, que é baseada numa combinação em cascata de três metodologias de controle. Para a malha interna, utiliza-se uma lei de controle para estabilizar os pólos localizados no lado direito do plano, em que serão testados um regulador quadrático linear (LQR, linear quadratic regulator em inglês) e um controlador $\mathscr{H}_{\infty}$ não-linear. Para a malha intermediária, utiliza-se um controlador baseado em linearização por realimentação ( $F L C$, em inglês) para desacoplar os pares de entrada/saída e, finalmente, para malha externa utiliza-se um controlador proporcionalderivativo - $P D$ para permitir o rastreamento de trajetória.

A Figura 5.1 exibe o diagrama de blocos ilustrando a estrutura de simulação, em que a sigla E.R. significa Estimador Robusto. O bloco do estimador fornece a estimativa das variáveis de controle definidas na expressão (5.1), enquanto o bloco intitulado Controlador representa o controlador que estabiliza a aeronave.

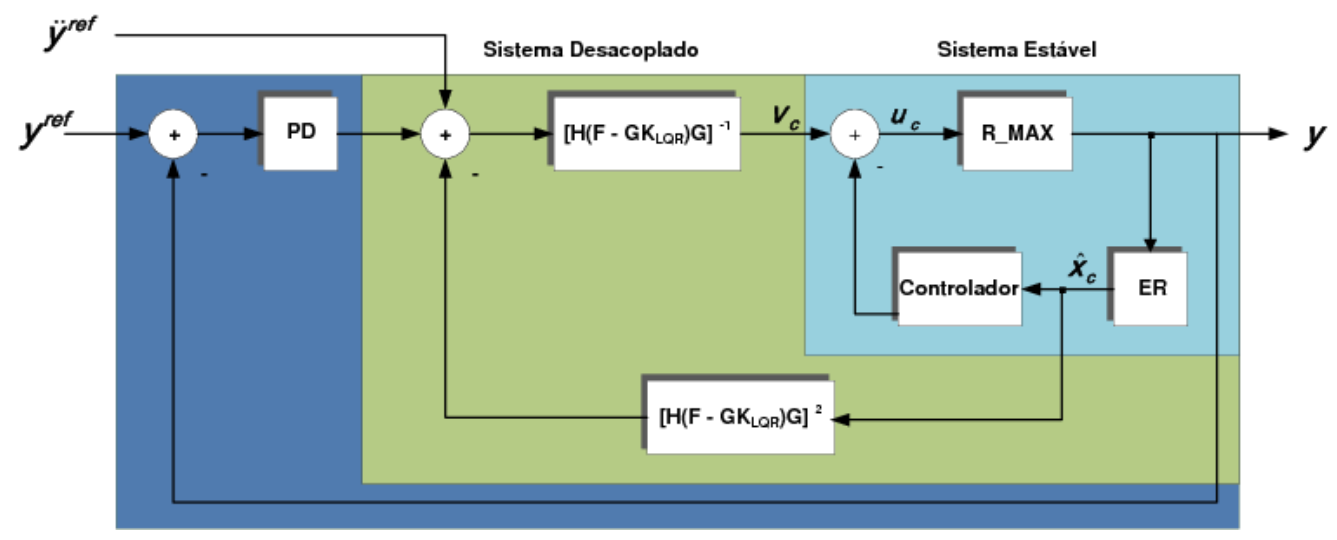

Figura 5.1: Diagrama de blocos do sistema.

A saída do estimador foi empregada como entrada para a etapa de controle. Para isso, utilizou-se um modelo reduzido, obtido a partir das Equações (4.7) e (4.9), eliminando-se as estimativas dos estados associados aos pólos menos dominantes do sistema. Tal modelo contém apenas posição, atitude, velocidades lineares e angulares e a taxa de realimentação da guinada 
[18]:

$$
\begin{array}{r}
\hat{\mathbf{x}}_{c}=\left[\begin{array}{lllllll}
x^{B} & y^{B} & z^{B} & u^{B} & v^{B} & w^{B} \\
\phi & \theta & \psi & p & q & r & r_{f b}
\end{array}\right]^{T}
\end{array}
$$

Desta forma, o modelo para o projeto de controle do helicóptero é dado por:

$$
\begin{aligned}
& \dot{\hat{\mathbf{x}}}_{\mathbf{c}}=A_{c} \hat{\mathbf{x}}_{\mathbf{c}}+B_{c} \mathbf{u}_{\mathbf{c}} \\
& \mathbf{y}_{\mathbf{c}}=C_{c} \hat{\mathbf{x}}_{\mathbf{c}}
\end{aligned}
$$

com

$$
\begin{aligned}
& \mathbf{u}_{\mathbf{c}}=\mathbf{u}=\left[\begin{array}{llll}
\delta_{l a t} & \delta_{l o n} & \delta_{c o l} & \delta_{p e d}
\end{array}\right]^{T}, \\
& \mathbf{y}_{\mathbf{c}}=\mathbf{y}=\left[\begin{array}{llll}
x^{B} & y^{B} & z^{B} & \psi
\end{array}\right]^{T} .
\end{aligned}
$$

A seguir serão abordadas cada etapa do sistema de controle.

\subsection{Malha Interna}

$\mathrm{O}$ vetor $K_{M I}$ é o ganho do controlador da malha interna, se referindo à lei de controle LQR ou à lei de controle $\mathscr{H}_{\infty}$, ambas sendo abordadas nas subseções a seguir.

\subsubsection{Regulador Quadrático Linear}

O LQR pertence à teoria de controle ótimo, campo que tem como objetivo a otimização (geralmente minimização) da energia de um sistema ou de uma função energia, quando o sistema parte de um estado inicial $\mathbf{x}\left(t_{0}\right)$ para um estado final $\mathbf{x}\left(t_{f}\right)$. Em particular, considere um sistema dinâmico descrito pela Equação (5.2), e suponha que o sistema precise alcançar um estado desejado $\mathbf{x}^{d}=\mathbf{0}$ enquanto o seguinte funcional de custo é minimizado:

$$
J=\int_{t_{0}}^{\infty}\left(\hat{\mathbf{x}}_{c}^{T} Q \hat{\mathbf{x}}_{c}+\mathbf{u}_{c}^{T} R \mathbf{u}_{c}\right) d t
$$

A metodologia LQR determina uma matriz de ganho fixo $K_{L Q R}$ tal que a lei de controle de realimentação de estado

$$
\mathbf{u}_{c}=-R^{-1} B^{T} P \hat{\mathbf{x}}_{c}
$$

minimize o funcional de custo enquanto o sistema alcance o estado desejado $\mathbf{x}^{d}$. Na prática, um 
dos efeitos de aplicar a metodologia $L Q R$ é que os pólos do sistema mudam daqueles dados pela matrix $A_{c}$ para aqueles dados por $A_{c}-B_{c} K_{L Q R}$. Desde que o sistema de controle seja estável sob a realimentação de estados via $L Q R$, pode-se utilizar outra metodologia de controle para guiar o sistema para qualquer saída admissível.

\subsubsection{Controlador $\mathscr{H}_{\infty}$ não-linear}

A lei de controle não-linear apresentada a seguir é baseada em Desigualdades Matriciais Lineares (DMLs). Trata-se de uma lei de controle por realimentação de estado $u=F(\rho) x$ que estabiliza o sistema em malha fechada garantindo que um ganho $\mathscr{L}_{2}$ entre o distúrbio e a saída seja limitado por um nível de atenuação $\gamma>0$.

\section{Ganho $\mathscr{L}_{2}$ para sistemas não-lineares variantes no tempo}

Considere um sistema não-linear variante no tempo com entrada de distúrbio afim $w \in \mathfrak{R}^{p}$ e saída controlada $z \in \mathfrak{R}^{q}$

$$
\begin{aligned}
& \dot{x}=f(x, t)+g(x, t) w \\
& z=h(x, t)+k(x, t) w
\end{aligned}
$$

sendo $f(0, t)=0$ e $h(0, t)=0$ para todo $t \in[0, T]$, e $x \in \mathfrak{R}^{n}$ o estado. Assume-se que $f(x, t)$, $g(x, t), h(x, t)$ e $k(x, t)$ são funções continuamente diferenciáveis em relação a $x$ e contínuas em t. O sistema (5.6) possui ganho $\mathscr{L}_{2} \leq \gamma$ no intervalo $[0, T]$ se

$$
\int_{0}^{T}\|z(t)\|^{2} d t \leq \gamma^{2} \int_{0}^{T}\|w(t)\|^{2} d t,
$$

para todo $T \geq 0$ e todo $w \in \mathscr{L}_{2}(0, T)$ com o sistema iniciando em $x(0)=0$. Para sistemas lineares invariantes no tempo, a condição de ganho $\mathscr{L}_{2} \leq \gamma$ corresponde à condição da norma $\mathscr{H}_{\infty}$ da função de transferência entre a entrada de distúrbio e a saída controlada ser limitada por $\gamma$, ou seja, $\left\|T_{z w}(s)\right\|_{\infty} \leq \gamma$.

\section{Síntese do controle $\mathscr{H}_{\infty}$ para sistemas LPV por realimentação do estado}

O problema consiste no controle por realimentação de estado dependente de parâmetro para estabilizar o sistema em malha fechada e fazer a norma $\mathscr{L}_{2}$ menor que um nível de desempenho 
especificado $\gamma$. Considere o problema de síntese do controle por realimentação do estado

$$
\begin{aligned}
\dot{x} & =A(\rho(t)) x+B_{1}(\rho(t)) w+B_{2}(\rho(t)) u \\
z_{1} & =C_{1}(\rho(t)) x \\
z_{2} & =C_{2}(\rho(t)) x+u
\end{aligned}
$$

sendo $x \in \mathfrak{R}^{n}$ o estado, $u \in \mathfrak{R}^{q_{4}}$ a entrada de controle, $w \in \mathfrak{R}^{p}$ o distúrbio de entrada, $z_{1} \in \mathfrak{R}^{q_{3}} \mathrm{e}$ $z_{2} \in \mathfrak{R}^{q_{4}}$ as saídas controladas, $A(\cdot), B_{1}(\cdot), B_{2}(\cdot), C_{1}(\cdot)$ e $C_{2}(\cdot)$ matrizes contínuas de dimensões apropriadas e $\rho(t) \in F_{P}^{v}$ definido por

$$
F_{P}^{v}=\left\{\rho \in \mathscr{C}^{1}\left(\Re^{+}, \Re^{m}\right): \rho(t) \in P,\left\|\dot{\rho}_{i}\right\| \leq v_{i}, i=1, \ldots, m\right\}
$$

sendo $P \subset \mathfrak{R}^{m}$ um conjunto compacto e $\mathrm{v}=\left[\mathrm{v}_{1} \cdots \mathrm{v}_{m}\right]^{T}$ com $\mathrm{v}_{i} \geq 0$.

Definição 5.1.1 [42] O problema de realimentação de estado dependente de parâmetro, para o sistema $L P V$ (5.8), é resolvido se existir uma função $Z \in C^{1}\left(\Re^{s}, S^{n \times n}\right)$ e uma $F \in C^{0}\left(\Re^{s} \times\right.$ $\left.\mathfrak{R}^{s}, \mathfrak{R}^{n_{u} \times n}\right)$ tais que para todo $\rho(t) \in P e\left\|\dot{\rho}_{i}\right\| \leq v_{i}, i=1,2, \ldots, s, Z(\rho)>0 e$

$$
\left[\begin{array}{cc}
A_{F}^{T}(\rho, \dot{\rho}) Z(\rho)+Z(\rho) A_{F}(\rho, \dot{\rho})+\sum_{i=1}^{s}\left(v_{i} \frac{\partial Z}{\partial \rho_{i}}\right)+C^{T}(\rho, \dot{\rho}) C(\rho, \dot{\rho}) & Z(\rho) B_{1}(\rho) \\
B_{1}^{T}(\rho) Z(\rho) & -\gamma^{2} I
\end{array}\right]<0,
$$

sendo $A_{F}(\rho, \dot{\rho}):=A(\rho)+B_{2}(\rho) F(\rho, \dot{\rho}), C(\rho, \dot{\rho}):=C_{1}(\rho)+D_{12} F(\rho, \dot{\rho})$ e $D_{12}=[0 I]^{T}$.

Se o problema de realimentação de estado dependente de parâmetro (5.9) tem solução, então a lei de controle $u=F(\rho(t)) x$ estabilizará exponencialmente o sistema em malha fechada e garantirá que a norma $\mathscr{L}_{2}$ induzida seja menor que $\gamma$. O seguinte teorema fornece uma condição de existência para o controlador de realimentação de estado expresso em DMLs para o sistema LPV em malha aberta (5.8).

Teorema 5.1.1 [42] Dado um conjunto compacto $P \in \mathfrak{R}^{s}$, um nível de desempenho $\gamma>0$ e o sistema (5.8), o problema de realimentação de estado tem solução se e somente se existir uma função $X \in C^{1}\left(\Re^{s}, S^{n \times n}\right)$ tal que para todo $\rho \in P, X(\rho)>0 e$

$$
\left[\begin{array}{ccc}
E(\rho) & X(\rho) C_{1}^{T}(\rho) & B_{1}(\rho) \\
C_{1}(\rho) X(\rho) & -I & 0 \\
B_{1}^{T}(\rho) & 0 & -\gamma^{2} I
\end{array}\right]<0
$$

sendo

$$
E(\rho)=-\sum_{i=1}^{m} \pm\left(v_{i} \frac{\partial X}{\partial \rho_{i}}\right)+\widehat{A}(\rho) X(\rho)+X(\rho) \widehat{A}(\rho)^{T}-B_{2}(\rho) B_{2}^{T}(\rho)
$$


$e \widehat{A}(\rho)=A(\rho)-B_{2}(\rho) C_{2}(\rho)$.

Este teorema fornece um ganho $F(\rho)$ de realimentação de estado tal que

$$
u=-\left(B_{2}(\rho)^{T} X^{-1}(\rho)+C_{2}(\rho)\right) x
$$

garante que o sistema em malha tenha ganho $\mathscr{L}_{2} \leq \gamma$ para toda variação paramétrica $\rho(t) \in F_{P}^{v}$.

O resultado acima é uma generalização natural da teoria de controle $\mathscr{H}_{\infty}$ para sistemas lineares, assumindo uma função de Lyapunov dependente de parâmetros na forma $V(x, t)=$ $x^{T}(t) X^{-1}(\rho(t)) x(t)$. Como resultado, deve-se resolver as DMLs paramétricas (5.10) que é um problema de otimização convexo com dimensão infinita.

\section{Cálculo do Controlador}

Um algoritmo prático para a obtenção do controlador [42, 43, 44, 45] é utilizado para resolver as desigualdades matriciais lineares presentes na análise e síntese dos problemas LPV.

Para determinar $X(\rho(t))$ na Equação (5.10), considere um conjunto de funções $\mathscr{C}^{1},\left\{f_{i}(\rho(t))\right\}_{i=1}^{M}$, como base para $X(\rho(t))$, ou seja,

$$
X(\rho(t))=\sum_{i=1}^{M} f_{i}(\rho(t)) X_{i}
$$

sendo $X_{i} \in S^{n \times n}$ a matriz coeficiente para $f_{i}(\rho(t))$. Substituindo $X(\rho(t))$, na Equação (5.10), por (5.12), o problema de realimentação de estado se transforma no seguinte problema de otimização

$$
\min _{\left\{X_{i}\right\}_{i=1}^{M}} \gamma^{2}
$$

sujeito a

$$
\left[\begin{array}{ccc}
E^{*}(\rho) & \sum_{j=1}^{M} f_{j}(\rho) X_{j} C_{1}^{T}(\rho) & B_{1}(\rho) \\
C_{1}(\rho) \sum_{j=1}^{M} f_{j}(\rho) X_{j} & -I & 0 \\
B_{1}^{T}(\rho) & 0 & -\gamma^{2} I
\end{array}\right]<0,
$$

sendo

$$
E^{*}(\rho)=-\sum_{i=1}^{M} \pm\left(v_{i} \sum_{j=1}^{M} \frac{\partial f_{j}}{\partial \rho_{i}} X_{j}\right)+\sum_{j=1}^{M} f_{j}(\rho)\left(\widehat{A}(\rho) X_{j}+X_{j} \widehat{A}(\rho)^{T}\right)-B_{2}(\rho) B_{2}^{T}(\rho)
$$


As DMLs (5.13) em termos das variáveis matriciais $\left\{X_{i}\right\}_{i=1}^{M}$ devem ser satisfeitas para todo parâmetro $\rho(t)$ em $P$. Este problema de otimização de dimensão infinita é resolvido ao se dividir o conjunto de parâmetros $P$ em $N$ pontos $\left\{\rho_{k}\right\}_{k=1}^{N}$ em cada dimensão, calculando as DMLs acima para estes pontos. Desde que a Equação (5.10) consiste de $2^{m}$ vínculos, um total de $\left(2^{m}+1\right) N^{m}$ desigualdades matriciais afins em termos das $M$ variáveis matriciais $\left\{X_{i}\right\}$ devem ser resolvidas e $\sum \pm(\cdot)$ indica que toda combinação $+(\cdot)$ e $-(\cdot)$ deve ser satisfeita. Uma aproximação da densidade de pontos particionados, $N$, que garante uma solução global das DMLs é dada por [42, 43].

Para este algoritmo, o número de parâmetros considerados e o número de divisões $N$ devem ser escolhidos de forma que a solução seja obtida em um número realizável de iterações.

\subsection{Malha Intermediária - Controlador de Linearização por Realimentação}

O controle através de linearização por realimentação consiste em determinar uma transformação e uma lei de realimentação visando linearizar o sistema e desacoplar os estados, permitindo o controle de cada saída independentemente das outras através de um simples controle linear. Como o sistema considerado é linear, este controlador foi utilizado apenas com o objetivo de desacoplar os estados e assim poder fechar a malha de controle com um controlador proporcional-derivativo. De um ponto de vista simplificado, a metodologia requer que se calcule sucessivas derivadas em relação ao tempo das saídas do sistema, até que todas as entradas apareçam explicitamente nas equações diferenciais resultantes. A esta altura, a equação é invertida e a entrada é calculada como uma função explícita das matrizes e estado do sistema.

Escolhe-se uma entrada de controle $\mathbf{u}_{c}$ como uma combinação da realimentação de estados $K_{M I}$ e uma entrada auxiliar $\mathbf{v}_{c}$ :

$$
\mathbf{u}_{c}=\mathbf{v}_{c}-K_{M I} \hat{\mathbf{x}}_{c}
$$

onde $K_{M I}$ é a matriz de ganho de realimentação, considerando o estado desejado $\mathbf{x}^{d}=\mathbf{0}$.

Susbstituindo a Equação (5.14) em (․2) e (5.3), tem-se

$$
\begin{aligned}
\dot{\hat{\mathbf{x}}}_{\mathbf{c}} & =\left(A_{c}-B_{c} K_{M I}\right) \hat{\mathbf{x}}_{c}+B_{c} \mathbf{v}_{c} \\
\mathbf{y}_{c} & =C_{c} \hat{\mathbf{x}}_{c}
\end{aligned}
$$


Diferenciando (5.16) e manipulando algebricamente, chega-se em:

$$
\begin{aligned}
\mathbf{v}_{c}= & {\left[C_{c}\left(A_{c}-B_{c} K_{M I}\right) B_{c}\right]^{-1} } \\
& \cdot\left[\overline{\mathbf{v}}_{c}-C_{c}\left(A_{c}-B_{c} K_{M I}\right)^{2} \dot{\hat{x}}_{c}\right],
\end{aligned}
$$

portanto o comportamento do laço fechado de $\mathbf{y}_{c}$ é ditado por $\ddot{\mathbf{y}}_{c}=\overline{\mathbf{v}}_{c}$.

\subsection{Malha Externa - Controlador PD}

Finalmente, escolhe-se $\overline{\mathbf{v}}_{c}$ como um controle proporcional-derivativo da forma

$$
\overline{\mathbf{v}}_{c}=\ddot{\mathbf{y}}_{\mathbf{c}}^{\text {ref }}+K_{d}\left(\dot{\mathbf{y}}_{c}^{r e f}-\dot{\mathbf{y}}_{c}\right)+K_{p}\left(\mathbf{y}_{c}^{r e f}-\mathbf{y}_{c}\right)
$$

em que $\mathbf{y}_{c}^{r e f}$ é a saída desejada. O comportamento dinâmico completo do erro $\mathbf{e}_{c}=\mathbf{y}_{c}^{r e f}-\mathbf{y}_{c}$ torna-se:

$$
\ddot{\mathbf{e}}_{c}+K_{d} \dot{\mathbf{e}}_{c}+K_{p} \mathbf{e}_{c}=\mathbf{0}
$$

Na ausência de erros de modelagem, uma seleção cuidadosa das matrizes de ganho $K_{p}$ e $K_{d}$ garante $\mathbf{e}_{c} \rightarrow \mathbf{0}$, ou seja, $\mathbf{y}_{c}$ converge para seu valor de referência com uma taxa de referência determinada pelos elementos das matrizes de ganho. Escolhendo matrizes diagonais e definidas positivas, cada saída é controlada independentemente das outras e sua dinâmica pode ser determinada arbitrariamente.

\subsection{Lei de Controle em Cascata}

O controlador final, portanto, é obtido pela combinação das estratégias descritas anteriormente:

$$
\mathbf{u}_{c}=\left[C_{c}\left(A c-B_{c} K_{M I}\right) B_{c}\right]^{-1}\left[\ddot{\mathbf{y}}_{\mathbf{c}}^{\text {ref }}+K_{d} \dot{\mathbf{e}}_{c}+K_{p} \mathbf{e}_{c}-C_{c}\left(A_{c}-B_{c} K_{M I}\right)^{2} \hat{\mathbf{x}}_{c}\right]-K_{M I} \hat{\mathbf{x}}_{c}
$$

\subsection{Seguidor de Waypoints em Sistema de Coordenadas do Corpo}

Retornando às variáveis $x^{B}, y^{B}, z^{B}$ presentes na estimativa do vetor $\hat{\mathbf{x}}_{c}$, elas representam a posição do helicóptero em um sistema de coordenadas que rotaciona juntamente com o corpo do helicóptero mas cuja origem é a mesma do sistema de coordenadas de navegação. Como 
guia de waypoints (destinos de um trecho de navegação) é geralmente definido no sistema de coordenadas de navegação, que não rotaciona mas é estritamente fixo na Terra, é necessário transformar os waypoints para o sistema de coordenadas do corpo no qual o controlador foi projetado. Para obter tal resultado, usa-se o fato de que a posição no sistema de coordenadas do corpo é igual a:

$$
\left[\begin{array}{c}
x^{B} \\
y^{B} \\
z^{B}
\end{array}\right]=R_{g}^{b}\left[\begin{array}{l}
x^{g} \\
y^{g} \\
z^{g}
\end{array}\right]
$$

em que $R_{g}^{b}$ é a matriz de transformação do sistema de navegação para corpo, dada pela Equação (2.6).

Portanto, o seguidor de waypoints em sistemas de coordenadas do corpo $x^{B}, y^{B}, z^{B}$ é alcançado utilizando como saída de referência

$$
\left(y_{c}^{r e f}\right)^{B}=R_{g}^{b}\left[\begin{array}{cccc} 
& & R_{g}^{b} & \\
0 & 0 & 0 & 1
\end{array}\right]\left(y_{c}^{r e f}\right)^{g}
$$




\section{$6 \quad$ Resultados}

Este capítulo se divide em duas seções: a primeira apresenta os resultados de simulação para a estimativa da posição e atitude utilizando os filtros descritos no Capítulo 3 e a segunda apresenta os resultados de simulação para cada uma das leis de controle da malha interna. Todos as simulações consideraram a Equação (5.3) como saída do sistema.

\subsection{Comparação entre Filtros}

O sistema utilizado para esta simulação é similar ao apresentado na Subseção 6.1, porém, compara-se o desempenho entre o filtro de Kalman padrão [46], o estimador robusto A (Seção 3.3) e o estimador robusto B (Seção 3.4).

Para simular o sistema proposto, foram feitas as seguintes considerações:

- As matrizes de estado $F$ e $G$ são afetadas por incertezas paramétricas;

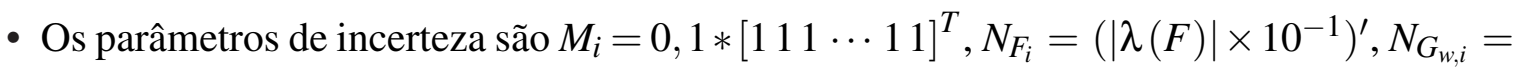
$\left(|\operatorname{svd}(G)| \times 10^{-3}\right)^{\prime}$. Frações de autovalores de $F$ e frações de valores singulares de $G$ foram escolhidos para representarem imperfeições no modelo;

- Os ruídos de estado e de medida são gaussianos e não correlacionados;

- O valor $\Delta_{i}$ é escalar e varia aleatoriamente em cada passo da simulação;

- Condições iniciais $P_{0 \mid 0}=\left(\Pi_{0}^{-1}+\left(H_{0}^{T} R_{0}{ }^{-1} H_{0}\right)^{-1}\right)^{-1}$ e $\hat{x}_{0}=P_{0 \mid 0} H_{0}^{T} R_{0}^{-1} y_{0}$;

- As trajetórias de referência foram geradas a partir de polinômios de $5^{a}$ ordem;

- Para o estimador robusto $\mathrm{A}, \alpha=1$;

- Para o estimador robusto B: $E_{i+1}=\mathbf{I}_{n}, G_{v, i+1}=\mathbf{0}_{n \times p},, K_{w, i}=\mathbf{0}_{p \times m}, K_{v, i+1}=\mathbf{I}_{p}, J_{i}=$ $\mathbf{0}_{p \times n}, N_{G_{w, i}} \approx \mathbf{0}_{1 \times m}, N_{G_{v, i+1}} \approx \mathbf{0}_{1 \times p}, N_{H_{i+1}} \approx \mathbf{0}_{1 \times n}, N_{E_{i+1}} \approx \mathbf{0}_{1 \times n}, N_{J_{i}}=\mathbf{0}_{1 \times n}, N_{K_{w, i}}=\mathbf{0}_{1 \times m}$, 
$N_{K_{v, i+1}} \approx \mathbf{0}_{1 \times p}, M_{2}=d t *\left[\begin{array}{llll}1 & 1 & \cdots & 1\end{array}\right]^{T}$ e $\mu=10000$. Os valores aproximados são utilizados por questões numéricas;

A simulação foi realizada no Matlab ${ }^{\circledR}$ da seguinte forma: cinquenta experimentos de cinquenta segundos para cada filtro com passo de dez milissegundos. O cálculo do erro é dado por:

$$
\varepsilon=\frac{1}{10} \sum_{i=1}^{10}\left\|x[k]-\hat{x}[k]^{(i)}\right\|
$$

onde $i$ corresponde ao número de experimentos, $k$ corresponde ao instante de tempo, $x$ é o estado e $\hat{x}$ corresponde à estimativa filtrada.

A seguir são apresentados os gráficos comparativos entre os desempenhos do filtro de Kalman nominal e dos estimadores robustos.

As Figuras 6.1 a, 6.1b e 6.1c exibem os resultados encontrados referentes à posição do helicóptero em cada eixo. Cada gráfico contém duas curvas que se referem: à trajetória do estado (linha sólida azul) e à trajetória da estimativa nominal (linha tracejada preta).

As Figuras 6.2 $\mathrm{a}, 6.2 \mathrm{~b}$ e 6.2 $\mathrm{k}$ exibem os gráficos das atitudes resultantes a partir do próprio estado e da estimativa nominal. Para estes casos, não há trajetórias de referência a serem seguidas, e os ângulos de rolagem e de guinada devem manter o intervalo de atuação limitado (por exemplo, $|\phi| \leq 10^{\circ}$ ).

As Figuras 6.3a, 6.3b e 6.3c exibem os resultados encontrados referentes à posição do helicóptero em cada eixo. Cada gráfico contém duas curvas que se referem: à trajetória do estado (linha sólida azul) e à trajetória da estimativa robusta A (linha tracejada preta).

As Figuras 6.4a, 6.4b e 6.4 exibem os gráficos das atitudes resultantes a partir do próprio estado e do filtro robusto A. As restrições de ângulos também se aplicam neste caso.

As Figuras 6.5a, 6.5b e 6.5c exibem os resultados encontrados referentes à posição do helicóptero em cada eixo. Cada gráfico contém duas curvas que se referem: à trajetória do estado (linha sólida azul) e à trajetória da estimativa robusta B (linha tracejada preta).

As Figuras 6.6a, 6.6 b e 6.6 exibem os gráficos das atitudes resultantes a partir do próprio estado e da estimativa robusta B. As restrições de ângulos também se aplicam neste caso.

A Tabela 6.1 exibe a comparação do desempenho de cada filtro segundo o erro calculado pela Equação 6.1).

De acordo com a Tabela 6.1 percebe-se que o erro da estimativa robusta A é cerca de 


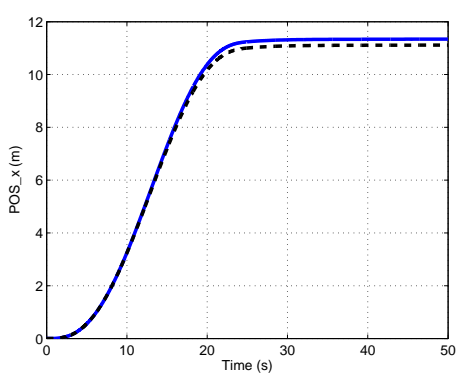

(a)

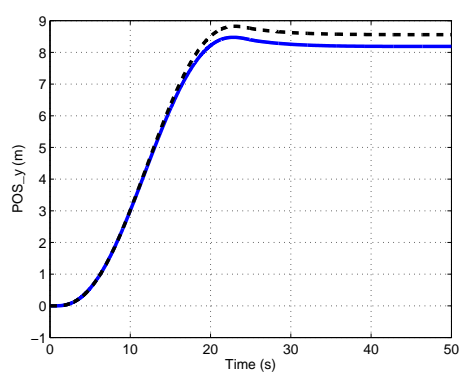

(b)

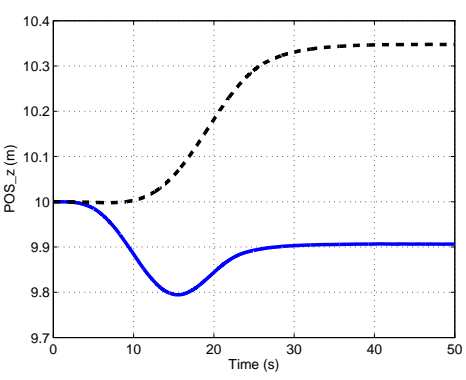

(c)

Figura 6.1: Comparação entre a posição da aeronave e a saída do filtro de Kalman: (a) Em x, (b) Em y, (c) Em z.

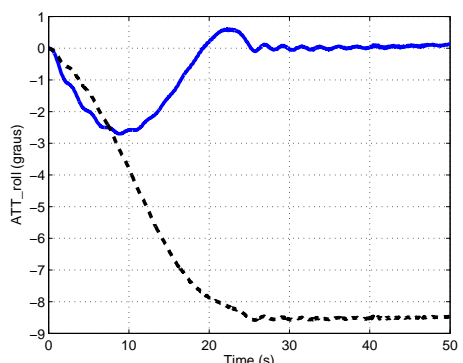

(a)

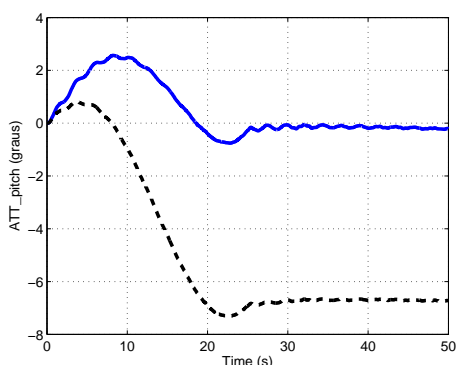

(b)

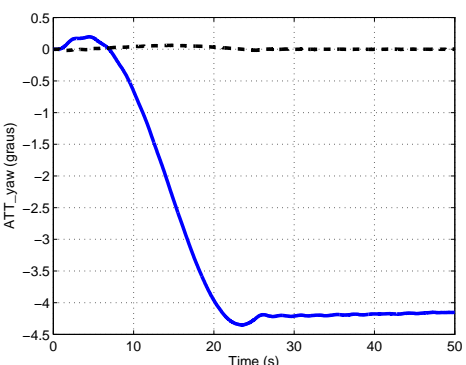

(c)

Figura 6.2: Comparação entre as atitudes e as saídas correspondentes do filtro de Kalman: (a) Rolagem, (b) Arfagem (c) Guinada.

\begin{tabular}{|c|c|}
\hline \multicolumn{1}{|c|}{ Filtro } & $\varepsilon$ \\
\hline Filtro Nominal & 1,1109 \\
\hline Estimativa Robusta A & 0,7045 \\
\hline Estimativa Robusta B & 0,7173 \\
\hline
\end{tabular}

Tabela 6.1: Desempenho de cada filtro de acordo com a Equação (6.1).

$36,58 \%$ menor que o erro do filtro nominal, enquanto que o erro da estimativa robusta B é cerca de $35,43 \%$ menor que o erro do filtro nominal. Note que em virtude de problemas numéricos no Filtro B nã foi considerado $\mu=10^{7}$ e não $\mu \rightarrow \infty$. Esses aspectos numéricos deverão ser tratados em trabalhos futuros.

\subsection{Comparação entre Sistemas de Controle da Malha In- terna}

Esta seção apresenta os resultados de simulação entre o regulador quadrático linear e o controlador $\mathscr{H}_{\infty}$ não-linear atuando na malha interna do controlador. Considerou-se que os dados estariam disponíveis para o controlador, portanto, não houve etapa de filtragem. 


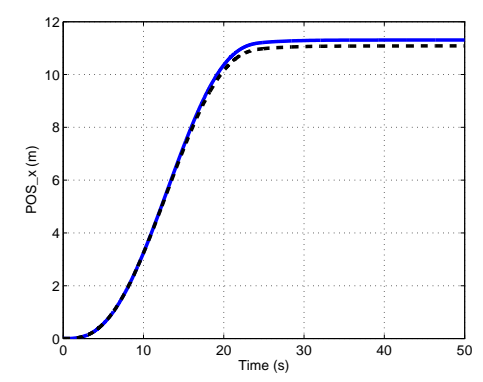

(a)

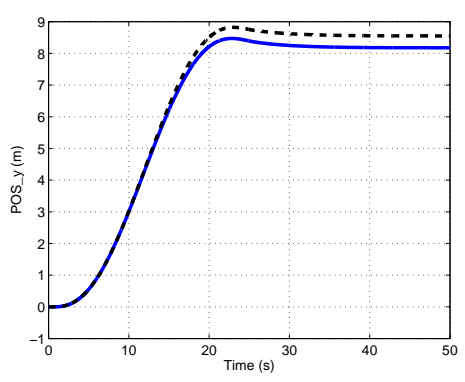

(b)

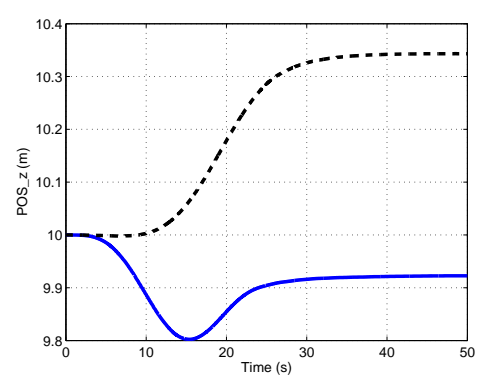

(c)

Figura 6.3: Comparação entre a posição da aeronave e a saída da estimativa robusta A: (a) Em $x$, (b) Em y, (c) Em z.

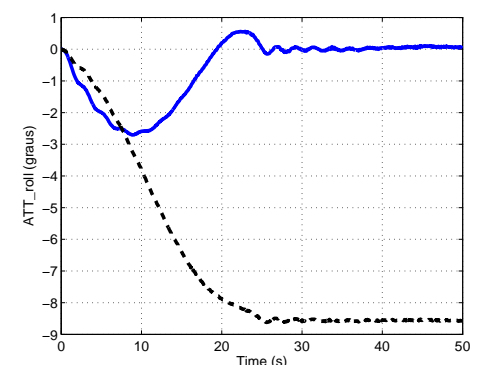

(a)

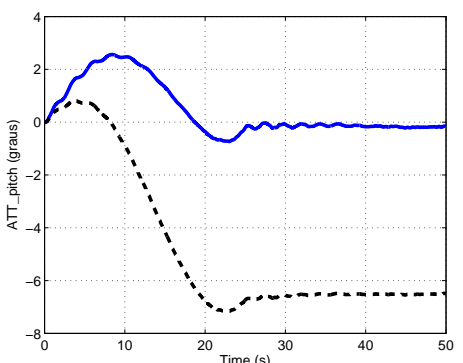

(b)

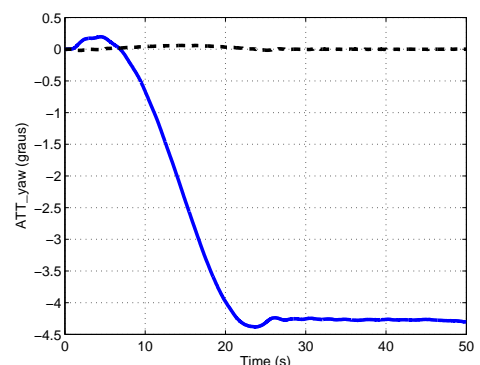

(c)

Figura 6.4: Comparação entre as atitudes e as saídas correspondentes da estimativa robusta A: (a) Rolagem, (b) Arfagem (c) Guinada.

Para verificar a robustez dos controladores, distúrbios externos do tipo senóide amortecida simulando rajadas de vento, iniciando em $t=10 \mathrm{~s}$, foram introduzidos artificialmente. Os distúrbios são dados por:

$$
\text { wind }=\left[\begin{array}{l}
W_{x} \exp ^{-2 t} \operatorname{sen}(2 \pi t) \\
W_{y} \exp ^{-2 t} \operatorname{sen}(2 \pi t) \\
W_{z} \exp ^{-2 t} \operatorname{sen}(2 \pi t)
\end{array}\right]
$$

sendo $W_{x}, W_{y}$ e $W_{z}$ as amplitudes dos distúrbios, em $m / s$, nas direções $x, y$ e $z$, respectivamente. A Figura 6.7 mostra os distúrbios utilizados, com $\left[\begin{array}{lll}W_{x} & W_{y} & W_{z}\end{array}\right]=\left[\begin{array}{lll}2 & 1 & 1\end{array}\right]$.

Para obter a lei de controle $\mathscr{H}_{\infty}$ não-linear, o conjunto compacto $P$ é definido por $\rho \in$ $[-30,30]^{\circ} \times[-30,30]^{\circ} \times[-90,90]^{\circ}$. A taxa de variação dos parâmetros é limitada a $|\dot{\rho}| \leq$ $60^{\circ} / \mathrm{s}$. As funções escolhidas para serem utilizadas como base para $X(\rho)$ foram: 


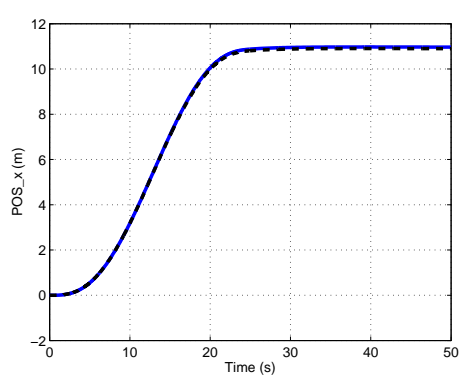

(a)

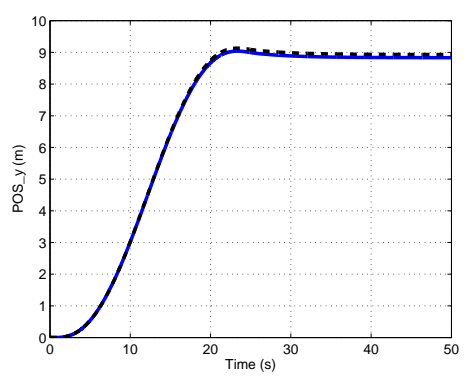

(b)

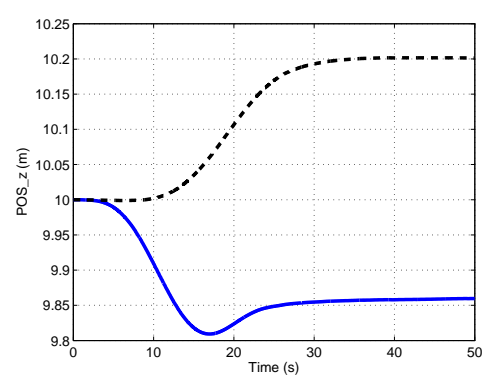

(c)

Figura 6.5: Comparação entre a posição da aeronave e a saída da estimativa robusta B: (a) Em $x$, (b) Em y, (c) Em z.

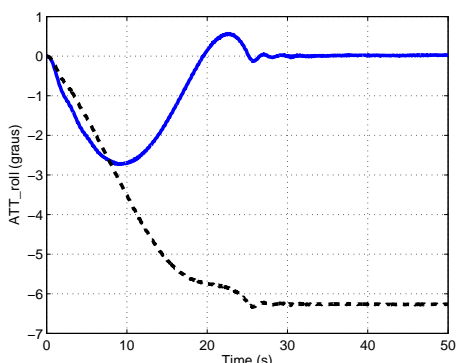

(a)

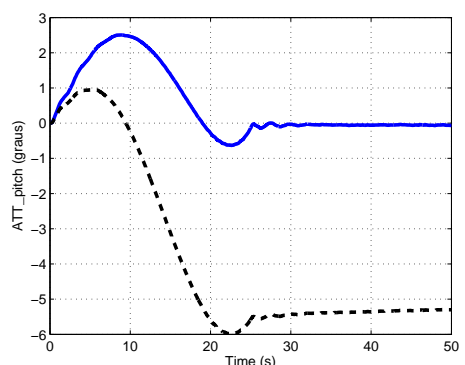

(b)

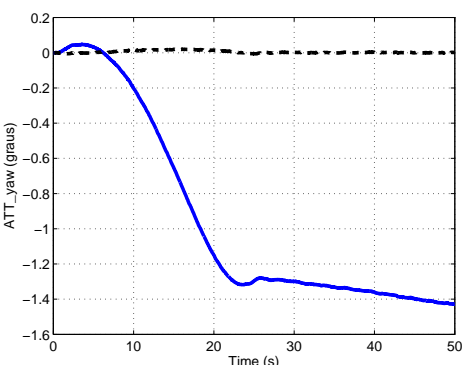

(c)

Figura 6.6: Comparação entre as atitudes e as saídas correspondentes da estimativa robusta B: (a) Rolagem, (b) Arfagem (c) Guinada.

$$
\begin{aligned}
& f_{1}(\rho(x))=15 \\
& f_{2}(\rho(x))=12 \cos \phi \\
& f_{3}(\rho(x))=7 \operatorname{sen} \theta \\
& f_{4}(\rho(x))=25 \operatorname{sen} \psi .
\end{aligned}
$$

O conjunto $P$ foi dividido em 3 para cada paramêtro $\phi, \theta$ e $\psi$. A desigualdade (5.10) foi resolvida utilizando o software MATLAB [47]. Sendo que, em (5.8), as matrizes $A(\rho(t))$, $B_{1}(\rho(t))$ e $B_{2}(\rho(t))$ são dadas por (4.7), (4.8) e (4.9). As matrizes $X_{i}$ foram determinadas com nível de atenuação de 1,25 .

Os ganhos das malhas externas de controle foram ajustados da maneira análoga à seção anterior: $K_{p}=\left\{\begin{array}{llll}1 & 1 & 30 & 50\end{array}\right\}, K_{d}=\left\{\begin{array}{llll}2 & 2 & 3 & 5\end{array}\right\}$. Além disso, foi utilizado um vetor de ganhos artificiais, $K_{u}=\{0,2 \quad 0,2 \quad 0,75 \quad 1\}$, para multiplicar cada elemento em $\mathbf{u}_{c}$ a fim de limitar a ação de controle [18].

A aeronave partiu da posição inicial $\{0 ; 0 ; 5\} \mathrm{m}$, com ângulo de direção $-12^{\circ}$, para a po- 


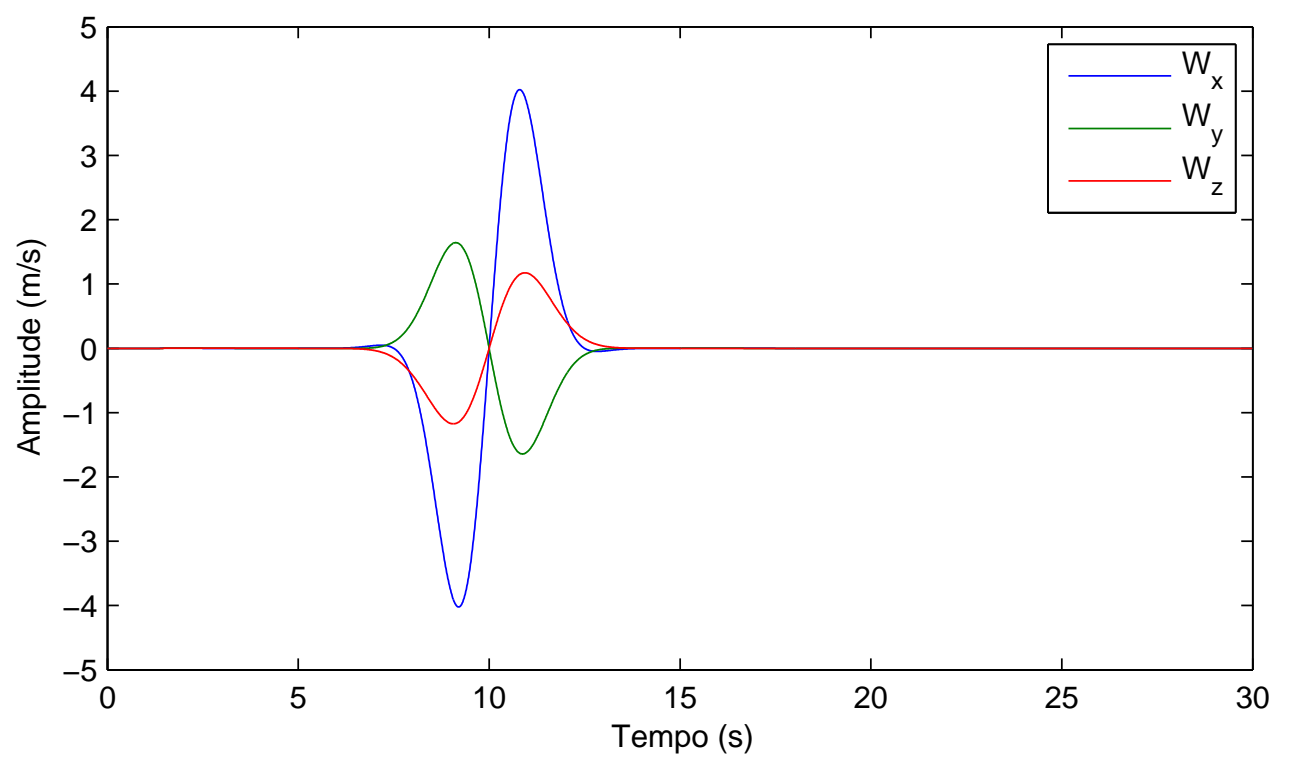

Figura 6.7: Distúrbios externos.

sição final $\{10 ; 10 ; 15\} \mathrm{m}$, com ângulo de direção $22^{\circ}$. A seguir são apresentados os gráficos comparativos entre os desempenhos do LQR e do controlador $\mathscr{H}_{\infty}$ não-linear.

As Figuras 6.8 $\mathrm{a}, 6.8 \mathrm{~b}$ e 6.8c exibem os resultados encontrados referentes à posição do helicóptero em cada eixo e as Figuras 6.9 a, 6.9 b e 6.9 $\mathrm{c}$ exibem os gráficos da atitude resultante.

Nesses gráficos relativos às posições e à direção $\psi$ as curvas que se referem: à trajetória de referência (linha sólida azul), à trajetória obtida através do LQR (linha sólida preta) e à trajetória obtida através do controlador $\mathscr{H}_{\infty}$ (linha traço-ponto vermelha). Para os ângulos $\phi$ e $\theta$, não há trajetórias de referência a serem seguidas. Os gráficos (a) e (b) possui duas curvas cada: trajetória obtida através do LQR (linha sólida preta) e à trajetória obtida através do controlador $\mathscr{H}_{\infty}$ (linha traço-ponto vermelha).

A Figura 6.10 exibe a variação da norma da matriz $A(\rho(t))$ durante a simulação.

A utilização do controlador $\mathscr{H}_{\infty}$ não-linear assumirá um papel mais importante, se comparado com o controlador linear, se for considerado um modelo matemático mais elaborado que considere, por exemplo, as características não-lineares da contribuição da propulsão dos rotores principal e de cauda. 


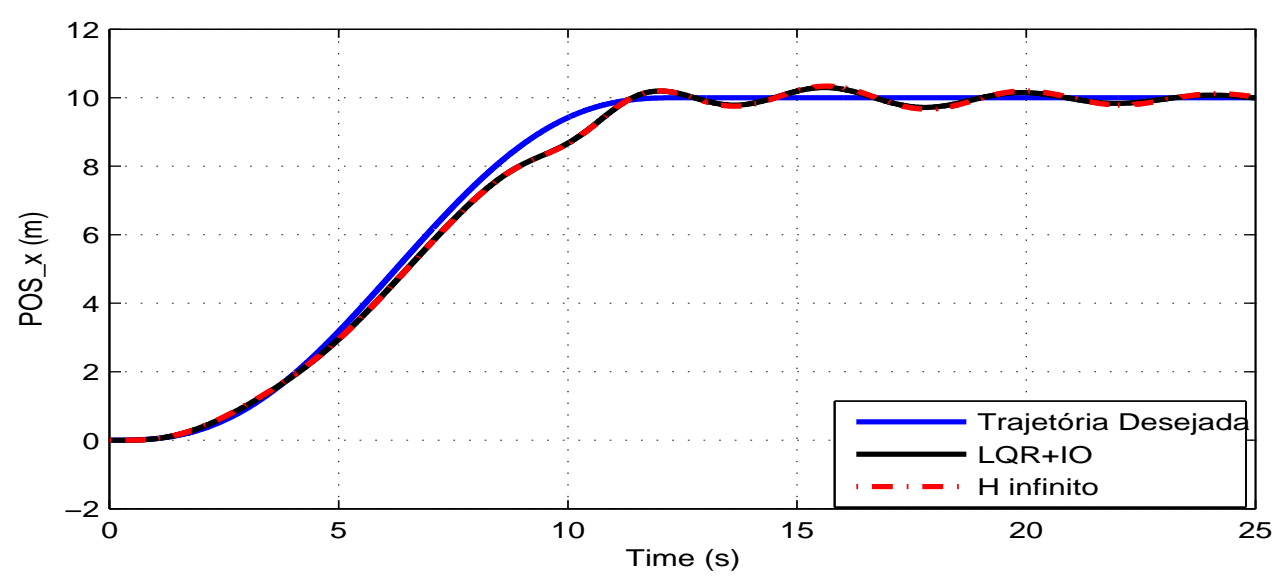

(a) Posição no eixo $x$.

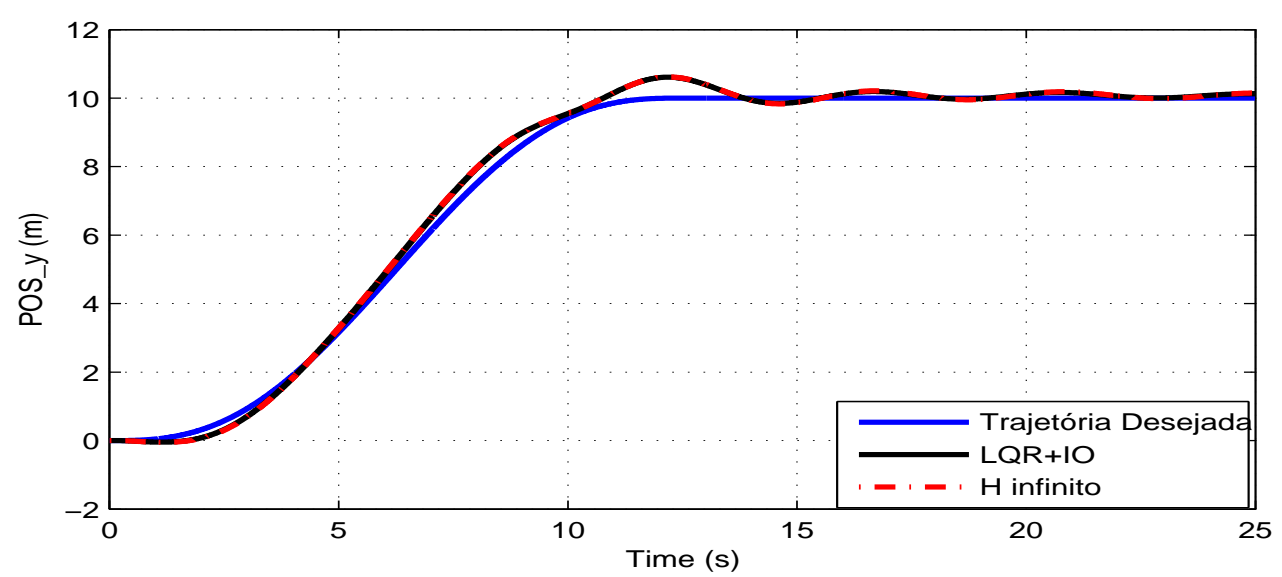

(b) Posição no eixo y.

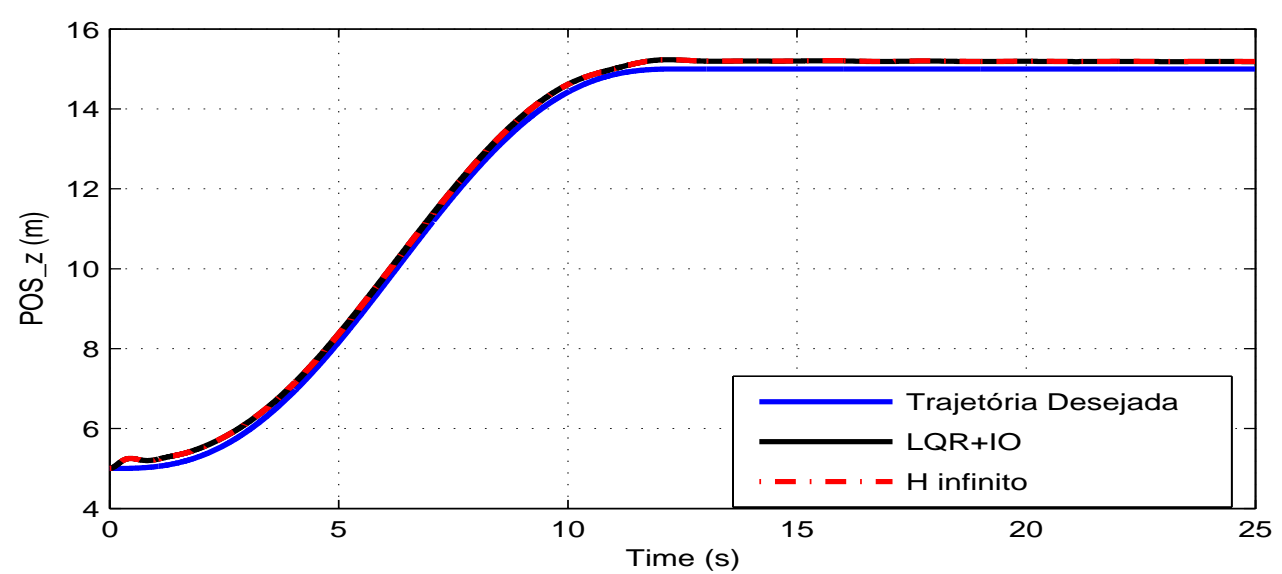

(c) Posição no eixo z.

Figura 6.8: Comparação entre a posição desejada da aeronave e a posição obtida, para distúbrio $\left[\begin{array}{lll}W_{x} & W_{y} & W_{z}\end{array}\right]=\left[\begin{array}{lll}2 & 1 & 1\end{array}\right]:(a)$ em $x,(b)$ em y e $(c)$ em $z$. 


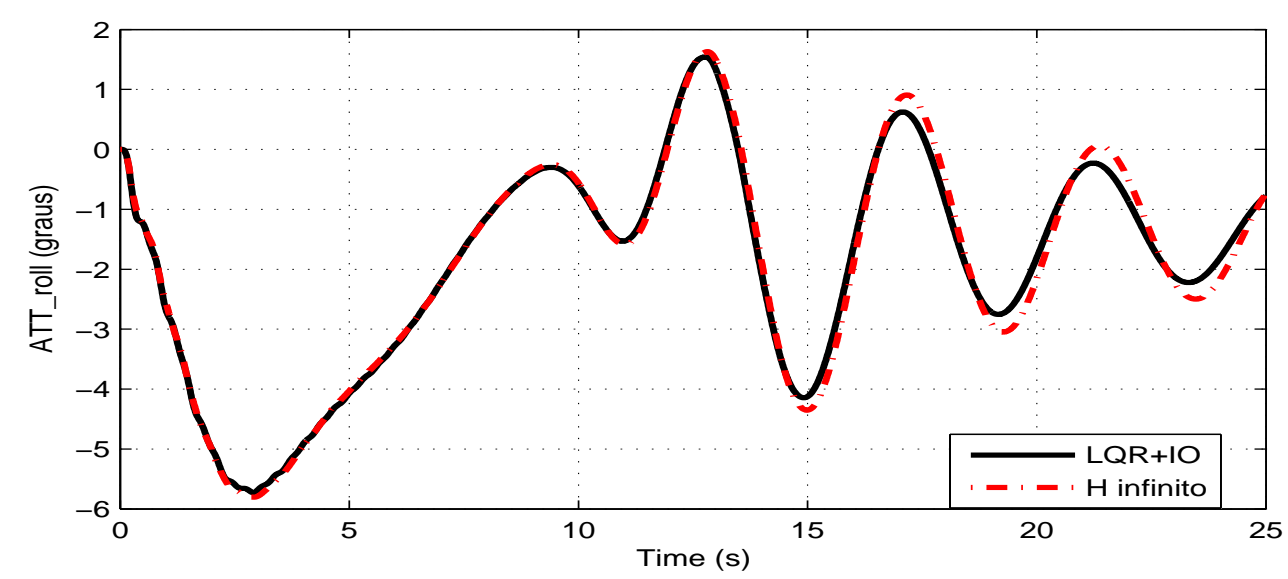

(a) Ângulo de rolagem.

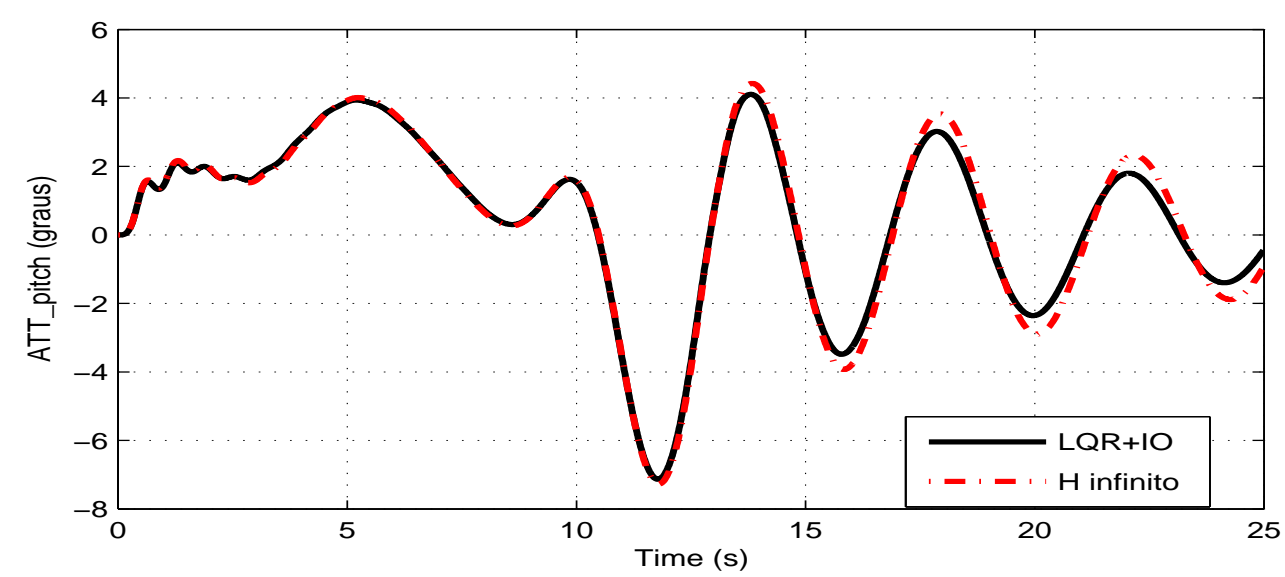

(b) Ângulo de arfagem.

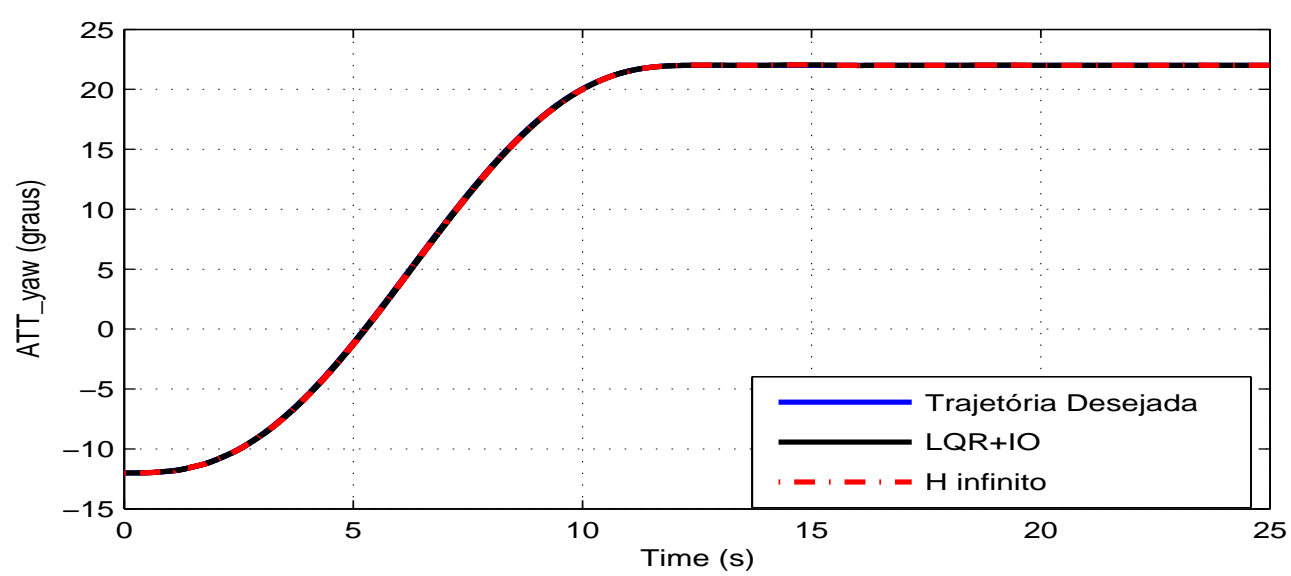

(c) Ângulo de guinada.

Figura 6.9: Comparação entre a atitude da aeronave para o distúrbio $\left[\begin{array}{lll}W_{x} & W_{y} & W_{z}\end{array}\right]=$ $\left[\begin{array}{lll}2 & 1 & 1\end{array}\right]$ : (a) rolagem, (b) arfagem e (c) guinada . 


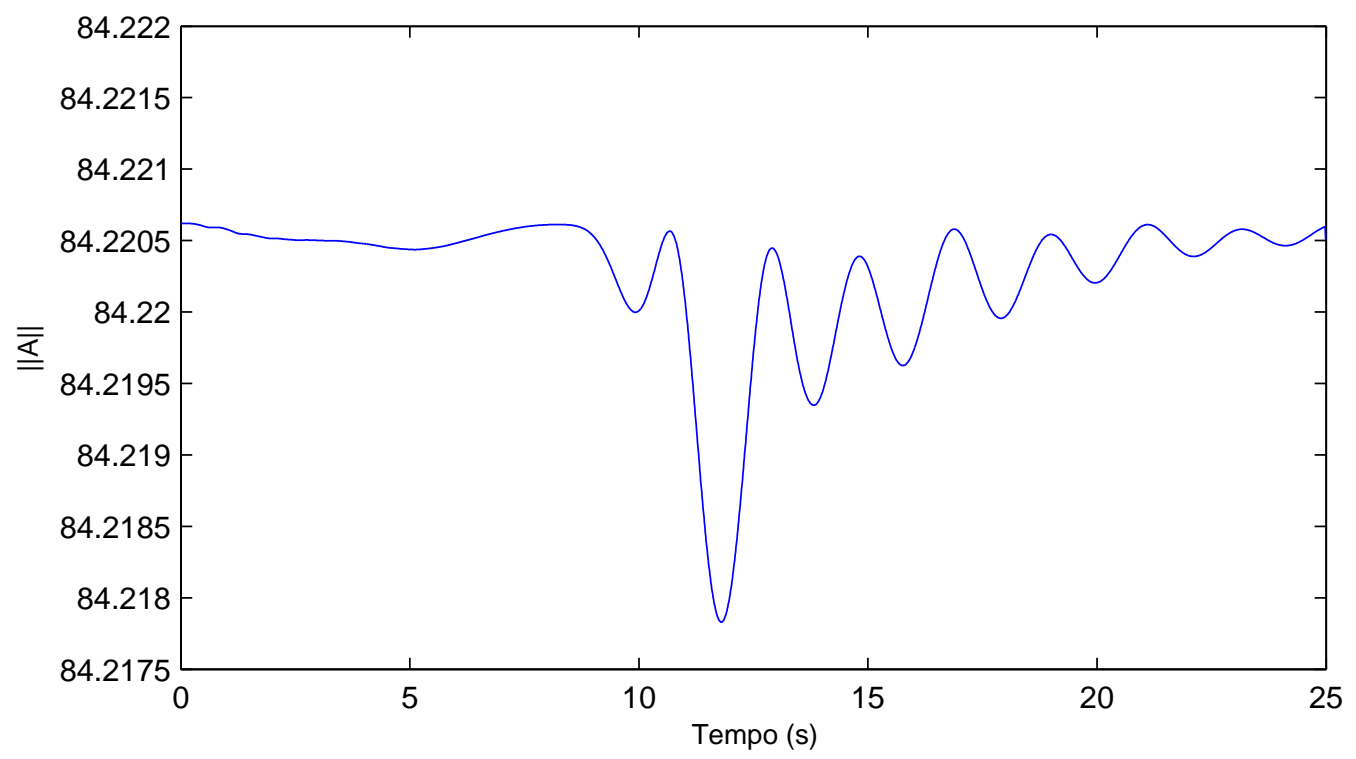

Figura 6.10: Variação da norma da matriz $A(\rho(t))$ durante o período de simulação. 


\section{Conclusão}

Este trabalho propôs o estudo de um sistema de referência inercial de posição e atitude para um helicóptero não-tripulado utilizando fusão de sensores através de filtragem. Filtros robustos foram aplicados com o intuito de minimizar os efeitos das incertezas nas estimativas dos estados de um helicóptero autônomo modelo Yamaha R-MAX. Os desempenhos da estimativa BDU e do filtro ótimo robuto foram comparados entre si e com uma formulação baseada no filtro de Kalman nominal.

Outro objetivo deste trabalho era utilizar o controlador $\mathscr{H}_{\infty}$ não-linear para estabilizar o helicóptero. Para isto, a matriz de parâmetros $A(\rho)$ do modelo em espaço de estado do helicópetro foi considerada linear com parâmetros variantes nos ângulos $\phi, \theta$ e $\psi$.

Como sugestão para trabalhos futuros, visando aproveitar o potencial do controlador $\mathscr{H}_{\infty}$ não-linear, deve-se utilizar modelos mais complexos que considerem as características nãolineares da contribuição da propulsão dos rotores principal e de cauda à dinâmica da aeronave.

Para o filtro ótimo robusto, Filtro B, a utilização de algoritmos que aproveitem a esparsidade da matriz a ser invertida para a obtenção da matriz de Riccati pode contribuir para melhorar o desempenho desse filtro. 


\section{Referências Bibliográficas}

[1] G. Cai, B. M. Chen, and T. H. Lee, "An overview on development fo miniature unmanned rotorcraft systems," Frontiers of Electrical and Electronic Engineering in China, vol. 5, no. 1 , pp. $1-14,2010$.

[2] R. P. Cheng, M. B. Tischler, and G. J. Schulein, "R-MAX helicopter state-space model identification for hover and forward-flight," Journal of the American Helicopter Society, vol. 51, no. 2, pp. 202 - 210, Abril 2006.

[3] V. Gavrilets, B. Mettler, and E. Feron, "Dynamic model for a miniature aerobatic helicopter,” MIT-LIDS report, no. LIDS-P-2580, Tech. Rep., 2003.

[4] E. D. Beckmann and G. A. Borges, "Nonlinear modeling, identification and control for a simulated miniature helicopter," in 5th Latin American Robotics Symposium, Salvador, BA, Brazil, 2008.

[5] Z. Zhou, J. Hu, S. Liu, and B. Chen, "System identification of UAV based on EWCLMS," in IMACS Multiconference on Computational Engineering in Systems Applications, 2006.

[6] A. Kallapur, M. Samal, V. Puttige, S. Anavatti, and M. Garratt, "A UKF-NN framework for system identification of small unmanned aerial vehicles," in Proceedings of the 11th International IEEE Conference on Intelligent Transportation Systems, Beijing, China, October 2008.

[7] O. Amidi, T. Kanade, , and J. R. Miller, "Vision-based autonomous helicopter research at Carnegie Mellon Robotics Institute 1991-1997," in American Helicopter Society International Conference, Heli, Japan, April 1998.

[8] H. Kim, D. Shim, and S. Sastry, "Flying robots: modeling, control and decision making," in Robotics and Automation, 2002. Proceedings. ICRA '02. IEEE International Conference on, vol. 1, 2002, pp. 66-71.

[9] V. Gavrilets, A. Shterenberg, M. Dahleh, and E. Feron, "Avionics system for a small unmanned helicopter performing aggressive maneuvers," in Proceedings of 19th Digital Avionics Systems Conference, 2000.

[10] R. D. Garcia and K. P. Valavanis, "The implementation of an autonomous helicopter testbed," Journal of Intelligent and Robotic Systems, vol. 54, no. 1 - 3, pp. 423-454, March 2009.

[11] H. Shim, T. Koo, F. Hoffmann, and S. Sastry, "A comprehensive study of control design for an autonomous helicopter," in Decision and Control, 1998. Proceedings of the 37th IEEE Conference on, Tampa, Florida, USA, December 1998. 
[12] R. Mahony, T. Hamel, and A. Dzul, "Hover control via Lyapunov control for an autonomous model helicopter," in Decision and Control, 1999. Proceedings of the 38th IEEE Conference on, Phoenix, Arizona, USA, December 1999.

[13] A. Isidori, L. Marconi, and A. Serran, "Robust nonlinear motion control of a helicopter," IEEE Transactions on Automatic Control, vol. 48, pp. 413-426, 2003.

[14] G. M. Voorsluijs, S. Bennani, and C. W. Scherer, "Linear and parameter-dependent robust control techniques applied to a helicopter UAV," in Proceedings of the 38-th AIAA Guidance, Navigation and Control Conference, Providence, RI; USA, 2004.

[15] A. Bogdanov, E. Wan, and G. Harvey, "SDRE flight control for X-CELL and R-MAX autonomous helicopters," in Decision and Control, 2004. CDC. 43rd IEEE Conference on, vol. 2, 2004, pp. $1196-1203$.

[16] R. D. Nardi, J. Togelius, O. E. Holland, and S. M. Lucas, "Evolution of neural networks for helicopter control: Why modularity matters," in Proceedings of the IEEE Congress on Evolutionary Computation, Vancouver, Canada, 2006.

[17] S. Saripalli and G. Sukhatme, "Landing a helicopter on a moving target," in Robotics and Automation, 2007 IEEE International Conference on, april 2007, pp. 2030 -2035.

[18] M. Bergerman, O. Amidi, J. R. Miller, N. Vallidis, and T. Dudek, "Cascaded position and heading control of a robotic helicopter," in Proceedings of the 2007 IEEE/RSJ International Conference on Intelligent Robots and Systems, San Diego, CA, USA, Oct - Nov 2007.

[19] L. Marconi and R. Naldi, "Robust full degree-of-freedom tracking control of a helicopter," Automatica, vol. 43, no. 11, pp. 1909 - 1920, 2007.

[20] K. Peng, G. Cai, B. M. Chen, M. Dong, K. Y. Lum, and T. H. Lee, "Design and implementation of an autonomous flight control law for a UAV helicopter," Automatica, vol. 45, no. 10, pp. $2333-2338,2009$.

[21] A. Bogdanov, M. Carlsson, G. Harvey, J. Hunt, R. Kieburtz, R. van der Merwe, and E. Wan, "State-dependent Riccati equation control of a small unmanned helicopter," in Proceedings of the AIAA Guidance, Navigation, and Control Conference and Exhibit, Austin, Texas, 2003.

[22] A. A. Bogdanov and E. A. Wan, "Model predictive neural control of a high-fidelity helicopter model," in Proceedings of the AIAA Guidance, Navigation, and Control Conference and Exhibit, Montreal, Canada, 2001.

[23] P. J. Garcia-Pardo, G. S. Sukhatme, and J. F. Montgomery, "Towards vision-based safe landing for an autonomous helicopter," Robotics and Autonomous Systems, vol. 38, no. 1, pp. 19-29, 2001.

[24] K. Harbick, J. F. Montgomery, and G. S. Sukhatme, "Planar spline trajectory following for an autonomous helicopter," Journal of Advanced Computational Intelligence - Computational Intelligence in Robotics and Automation, vol. 8, no. 3, pp. 237-242, May 2004. 
[25] L. O. Mejias, S. Saripalli, P. Cervera, and G. S. Sukhatme, "Visual servoing of an autonomous helicopter in urban areas using feature tracking," Journal of Field Robotics, vol. 23, no. 3, pp. 185-199, 2006.

[26] K. Natesan, D.-W. Gu, I. Postlethwaite, and J. Chen, "Design and flight controllers based on simplified LPV model of a UAV," in Proceedings of the 45th IEEE Conference on Decision and Control, San Diego, CA, USA, December 2006.

[27] E. A. Wan and A. A. Bogdanov, "Model predictive neural control with applications to a 6 dof helicopter model," in Proceedings of the American Control Conference, Arlington, $V A, 2001$.

[28] E. A. Wan, A. A. Bogdanov, R. Kieburtz, A. Baptista, M. Carlsson, Y. Zhang, and M. Zulauf, Software Enabled Control: Information Technologies for Dynamical Systems. IEEE Press, Wiley \& Sons, 2003, ch. Model Predictive Neural Control for Aggressive Helicopter Maneuvers.

[29] C. Hide, T. Moore, and M. Smith, "Adaptive Kalman filtering algorithms for integrating GPS and low cost INS," in Position Location and Navigation Symposium, 2004. PLANS 2004, April 2004, pp. 227-233.

[30] T. Gao, Z. Gong, J. Luo, W. Ding, and W. Feng, "An attitude determination system for a small unmanned helicopter using low-cost sensors," Robotics and Biomimetics, IEEE International Conference on, vol. 0, pp. 1203-1208, 2006.

[31] J. M. Pflimlin, T. Hamel, and P. Souères, "Nonlinear attitude and gyroscope's bias estimation for a VTOL UAV," International Journal of Systems Science, vol. 38, no. 3, pp. 197 -210, March 2007.

[32] N. Abdelkrim, N. Aouf, A. Tsourdos, and B. White, "Robust nonlinear filtering for INS/GPS UAV localization," in 16th Mediterranean Conference on Control and Automation, Ajaccio, France, June 2008.

[33] Z. Xiaochuan, L. Qingsheng, H. Baoling, and L. Xiyu, "A novel information fusion algorithm for GPS/INS navigation system," in Proceedings of the 2009 IEEE International Conference on Information and Automation, Zhuhai/Macau, China, June 2009.

[34] A. H. Sayed, "A framework for state-space estimation with uncertain models," IEEE Trans. on Automatic Control, vol. 46, no. 7, pp. 998-1013, 2001.

[35] A. F. Bianco, "Filtros de Kalman robustos para sistemas dinâmicos singulares em tempo discreto," Ph.D. dissertation, Escola de Engenharia de São Carlos - USP, 2009.

[36] J. A. Farrel, Aided Navigation GPS with High Rate Sensors. McGraw-Hill Professional, 2008.

[37] L. Xia, J. Wang, and G. Yan, "RBFNN aided extended Kalman filter for MEMS AHRS/GPS," in Embedded Software and Systems, 2009. ICESS '09. International Conference on, May 2009, pp. 559-564.

[38] R. S. Inoue, M. H. Terra, A. A. G. Siqueira, and J. M. S. C. Yabarrena, "Filtragem robusta para navegação inercial," in Simpósio Brasileiro de Automação Inteligente, Setembro 2009. 
[39] J. Farrel and M. Barth, The global positioning system and inertial navigation, S. Chapman, Ed. McGraw-Hill, 1998.

[40] M. B. Tischler and R. Remple, Aircraft And Rotorcraft System Identification, Engineering Methods With Flight-test Examples. AIAA Education Series, 2006.

[41] D. F. de A. Lopes, M. H. Terra, and M. Bergerman, "Controle de helicóptero autonomo baseado em filtragem robusta," in Simpósio Brasileiro de Automação Inteligente, Setembro 2009.

[42] F. Wu, "Control of linear parameter-varying systems." Ph.D. dissertation, Department of Mechanical Engineering, University of California, Berkeley, 1995.

[43] F. Wu, X. H. Yang, A. Packard, and G. Becker, "Induced $\mathscr{L}_{2}$-norm control for lpv systems with bounded parameter variation rates," International Journal of Robust and Nonlinear Control, vol. 6, no. 9-10, pp. 983-998, 1996.

[44] Y. Huang and A. Jadbabaie, "Nonlinear $\mathscr{H}_{\infty}$ control: An enhanced quasi-lpv approach," in IEEE International Conference on Decision and Control, 37, Tampa, Florida, USA. Workshop in $\mathscr{H}_{\infty}$ nonlinear control by J. C. Doyle, Caltech, 1998.

[45] A. A. G. Siqueira and M. H. Terra, "Nonlinear and markovian $\mathscr{H}_{\infty}$ controls of underactuated manipulators," IEEE Transactions on Control System Tecnology, vol. 12, no. 6, pp. 811-826, November 2004.

[46] T. Kailath, A. Sayed, and B. Hassibi, Linear Estimation. Prentice Hall, 1999.

[47] R. S. Inoue, "Controle robusto de robôs móveis com rodas," Master's thesis, Universidade de São Paulo, 2007.

[48] J. B. Kuipers, Quaternions and rotation sequences. Princenton, New Jersey: Pricenton University Press, 1998. 


\section{APÊEDICE A - Quatérnios}

Conforme mostrado em [48], o quatérnio foi desenvolvido por William Rowan Hamilton em 1843 como uma generalização dos números complexos. Ele é representado por quatro parâmetros de números reais $\left(q_{0}, q_{1}, q_{2}, q_{3}\right)$ e pode ser definido pela soma:

$$
q=q_{0}+\mathbf{q}=q_{0}+q_{1} \mathbf{i}+q_{2} \mathbf{j}+q_{3} \mathbf{k},
$$

sendo $q_{0}$ e $\mathbf{q}$ a parte escalar e vetorial do quatérnio, respectivamente.

A respeito de operações de quatérnios, tem-se o seguinte:

1. Dois quatérnios $p=p_{0}+p_{1} \mathbf{i}+p_{2} \mathbf{j}+p_{3} \mathbf{k}$ e $q=q_{0}+q_{1} \mathbf{i}+q_{2} \mathbf{j}+q_{3} \mathbf{k}$ são iguais se, e somente se, os seus componentes são exatamente os mesmos. Ou seja, $p=q$ se, e somente se,

$$
p_{0}=q_{0}, p_{1}=q_{1}, p_{2}=q_{2}, p_{3}=q_{3}
$$

2. A soma de dois quatérnios $p$ e $q$ acima é definida pela adição dos componentes correspondentes:

$$
p+q=\left(p_{0}+q_{0}\right)+\left(p_{1}+q_{1}\right) \mathbf{i}+\left(p_{2}+q_{2}\right) \mathbf{j}+\left(p_{3}+q_{3}\right) \mathbf{k}
$$

3. O produto de um escalar $c$ e um quatérnio $q$ é dado por

$$
c q=c q_{0}+c q_{1} \mathbf{i}+c q_{2} \mathbf{j}+c q_{3} \mathbf{k}
$$


4. O produto de dois quatérnios é definido pelos produtos dos elementos de base:

$$
\begin{aligned}
\mathbf{i}^{2} & =\mathbf{j}^{2}=\mathbf{k}^{2}=\mathbf{i j k}=-1, \\
\mathbf{i j} & =\mathbf{k}=-\mathbf{j i} \\
\mathbf{j} \mathbf{k} & =\mathbf{i}=-\mathbf{k} \mathbf{j} \\
\mathbf{k i} & =\mathbf{j}=-\mathbf{i k} .
\end{aligned}
$$

Assim, tem-se

$$
\begin{aligned}
p \otimes q & =\left(p_{0}+p_{1} \mathbf{i}+p_{2} \mathbf{j}+p_{3} \mathbf{k}\right)\left(q_{0}+q_{1} \mathbf{i}+q_{2} \mathbf{j}+q_{3} \mathbf{k}\right) \\
& =p_{0} q_{0}-\left(p_{1} q_{1}+p_{2} q_{2}+p_{3} q_{3}\right) \\
& +p_{0}\left(q_{1} \mathbf{i}+q_{2} \mathbf{j}+q_{3} \mathbf{k}\right)+q_{0}\left(p_{1} \mathbf{i}+p_{2} \mathbf{j}+p_{3} \mathbf{k}\right) \\
& +\left(p_{2} q_{3}-p_{3} q_{2}\right) \mathbf{i}+\left(p_{3} q_{1}-p_{1} q_{3}\right) \mathbf{j}+\left(p_{1} q_{2}-p_{2} q_{1}\right) \mathbf{k}
\end{aligned}
$$

sendo $\otimes$ definido como a multiplicação entre dois quatérnios.

Lembrando o produto escalar e o produto vetorial da álgebra de vetores em três dimensões, têm-se que, para os vetores

$$
\mathbf{a}=\left(a_{1}, a_{2}, a_{3}\right) \text { e } \mathbf{b}=\left(b_{1}, b_{2}, b_{3}\right)
$$

o produto escalar é dado por:

$$
\mathbf{a} \cdot \mathbf{b}=a_{1} b_{1}+a_{2} b_{2}+a_{3} b_{3}
$$

e o produto vetorial é:

$$
\begin{aligned}
\mathbf{a} \times \mathbf{b} & =\left|\left[\begin{array}{ccc}
\mathbf{i} & \mathbf{j} & \mathbf{k} \\
a_{1} & a_{2} & a_{3} \\
b_{1} & b_{2} & b_{3}
\end{array}\right]\right|=\left(a_{2} b_{3}-a_{3} b_{2}\right) \mathbf{i}+\left(a_{3} b_{1}-a_{1} b_{3}\right) \mathbf{j}+\left(a_{1} b_{2}-a_{2} b_{1}\right) \mathbf{k} \\
& =\left[\begin{array}{ccc}
0 & -a_{3} & a_{2} \\
a_{3} & 0 & -a_{1} \\
-a_{2} & a_{1} & 0
\end{array}\right] \mathbf{b}=\left[\mathbf{a}^{\times}\right] \mathbf{b}
\end{aligned}
$$


forma:

$$
\begin{aligned}
p \otimes q & =p_{0} q_{0}-\mathbf{p} \cdot \mathbf{q}+p_{0} \mathbf{q}+q_{0} \mathbf{p}+\mathbf{p} \times \mathbf{q} \\
& =\left[\begin{array}{c}
p_{0} q_{0}-\mathbf{p} \cdot \mathbf{q} \\
p_{0} \mathbf{q}+q_{0} \mathbf{p}+\mathbf{p} \times \mathbf{q}
\end{array}\right] \\
& =\left[\begin{array}{cccc}
p_{0} & -p_{1} & -p_{2} & -p_{3} \\
p_{1} & p_{0} & -p_{3} & p_{2} \\
p_{2} & p_{3} & p_{0} & -p_{1} \\
p_{3} & -p_{2} & p_{1} & p_{0}
\end{array}\right]\left[\begin{array}{c}
q_{0} \\
q_{1} \\
q_{2} \\
q_{3}
\end{array}\right] .
\end{aligned}
$$

5. O conjugado complexo de um quatérnio é definido por

$$
q^{*}=q_{0}-\mathbf{q}=q_{0}-q_{1} \mathbf{i}-q_{2} \mathbf{j}-q_{3} \mathbf{k}
$$

6. O módulo do quatérnio é definido por

$$
|q|=\sqrt{q \otimes q^{*}}=\sqrt{q_{0}^{2}+q_{1}^{2}+q_{2}^{2}+q_{3}^{2}}
$$

7. Todo quatérnio diferente de zero tem um multiplicativo inverso. Pela definição de inversa, tem-se

$$
q^{-1} \otimes q=q \otimes q^{-1}=1
$$

Assim, pré e pós multiplicando pelo complexo conjugado $q^{*}$, pode-se escrever:

$$
q^{-1} \otimes q \otimes q^{*}=q^{*} \otimes q \otimes q^{-1}=q^{*}
$$

E desde que $q \otimes q^{*}=|q|^{2}$, tem-se:

$$
q^{-1}=\frac{q^{*}}{|q|^{2}}
$$

Perceba que se $q$ é um quatérnio unitário ou normalizado, $|q|=1$, então a inversa é simplesmente o complexo conjugado

$$
q^{-1}=q^{*}
$$




\section{A.1 Considerações geométricas}

Sabe-se que

$$
\cos ^{2} \frac{\theta}{2}+\operatorname{sen}^{2} \frac{\theta}{2}=1 .
$$

Então, desde que

$$
q_{0}^{2}+|\mathbf{q}|^{2}=1
$$

pode-se chamar

$$
\cos ^{2} \frac{\theta}{2}=q_{0}^{2}
$$

$\mathrm{e}$

$$
\operatorname{sen}^{2} \frac{\theta}{2}=|\mathbf{q}|^{2}
$$

para $\frac{\theta}{2}$ que satisfaz a restrição

$$
-\pi<\frac{\theta}{2} \leq \pi
$$

Dessa forma, definindo um vetor unitário u que representa a direção de $\mathbf{q}$

$$
\mathbf{u}=\frac{\mathbf{q}}{|\mathbf{q}|}=\frac{\mathbf{q}}{\operatorname{sen} \frac{\theta}{2}}
$$

pode-se escrever o quatérnio unitário $q$ em função do ângulo $\frac{\theta}{2}$ e do vetor unitário u como

$$
q=q_{0}+\mathbf{q}=\cos \frac{\theta}{2}+\operatorname{sen} \frac{\theta}{2} \mathbf{u}
$$

Desse modo, o quatérnio $q$ será sempre um quatérnio unitário ou normalizado, ou seja, com módulo 1

$$
q_{0}^{2}+|\mathbf{q}|^{2}=1
$$

Observe que, substituindo $\frac{\theta}{2}$ por $-\frac{\theta}{2}$, obtém-se o complexo conjugado de $q$, dado por:

$$
\begin{aligned}
\cos \left(-\frac{\theta}{2}\right)+\operatorname{sen}\left(-\frac{\theta}{2}\right) \mathbf{u} & =\cos \frac{\theta}{2}+\left(-\operatorname{sen} \frac{\theta}{2}\right) \mathbf{u} \\
& =\cos \frac{\theta}{2}-\operatorname{sen} \frac{\theta}{2} \mathbf{u}=q^{*} .
\end{aligned}
$$


Assim, por convenção, o quatérnio

$$
q=q_{0}+\mathbf{q}
$$

é representado por

$$
q=\cos \frac{\theta}{2}+\operatorname{sen} \frac{\theta}{2} \mathbf{u}
$$

\section{A.2 Operador quatérnio de rotação}

O resultado da rotação $q$ (representado como um quatérnio unitário) sobre algum ponto $\mathbf{x}$ (representado como um vetor) é dado por:

$$
\begin{aligned}
\mathbf{w} & =q \otimes\left[\begin{array}{l}
0 \\
\mathbf{v}
\end{array}\right] \otimes q^{*} \\
& =\left(q_{0}+\mathbf{q}\right)(0+\mathbf{v})\left(q_{0}-\mathbf{q}\right) \\
& =\left(q_{0} \mathbf{v}+\mathbf{q v}\right)\left(q_{0}-\mathbf{q}\right) \\
& =q_{0}^{2} \mathbf{v}-q_{0} \mathbf{v q}+q_{0} \mathbf{q} \mathbf{v}-\mathbf{q v q} \\
& =q_{0}^{2} \mathbf{v}-q_{0}(-\mathbf{v} \cdot \mathbf{q}+\mathbf{v} \times \mathbf{q})+q_{0}(-\mathbf{q} \cdot \mathbf{v}+\mathbf{q} \times \mathbf{v})-(-\mathbf{q} \cdot \mathbf{v}+\mathbf{q} \times \mathbf{v}) \mathbf{q} \\
& =q_{0}^{2} \mathbf{v}+q_{0}(\mathbf{v} \cdot \mathbf{q})-q_{0}(\mathbf{v} \times \mathbf{q})-q_{0}(\mathbf{q} \cdot \mathbf{v})+q_{0}(\mathbf{q} \times \mathbf{v})+(\mathbf{q} \cdot \mathbf{v}) \mathbf{q}-(\mathbf{q} \times \mathbf{v}) \mathbf{q} \\
& =q_{0}^{2} \mathbf{v}+2 q_{0}(\mathbf{q} \times \mathbf{v})+(\mathbf{q} \cdot \mathbf{v}) \mathbf{q}-(\mathbf{q} \times \mathbf{v}) \mathbf{q} \\
& =q_{0}^{2} \mathbf{v}+2 q_{0}(\mathbf{q} \times \mathbf{v})+(\mathbf{q} \cdot \mathbf{v}) \mathbf{q}+(\mathbf{q} \times \mathbf{v}) \cdot \mathbf{q}-\mathbf{q} \times \mathbf{v} \times \mathbf{q}
\end{aligned}
$$

Como $(\mathbf{q} \times \mathbf{v}) \cdot \mathbf{q}=0$ e $\mathbf{q} \times \mathbf{v} \times \mathbf{q}=(\mathbf{q} \cdot \mathbf{q}) \mathbf{v}-(\mathbf{q} \cdot \mathbf{v}) \mathbf{q}$, o operador $\mathbf{w}$ torna-se

$$
\begin{aligned}
\mathbf{w} & =q_{0}^{2} \mathbf{v}+2 q_{0}(\mathbf{q} \times \mathbf{v})+(\mathbf{q} \cdot \mathbf{v}) \mathbf{q}-(\mathbf{q} \cdot \mathbf{q}) \mathbf{v}+(\mathbf{q} \cdot \mathbf{v}) \mathbf{q} \\
& =\left(q_{0}^{2}-\mathbf{q} \cdot \mathbf{q}\right) \mathbf{v}+2(\mathbf{q} \cdot \mathbf{v}) \mathbf{q}+2 q_{0}(\mathbf{q} \times \mathbf{v}) \\
& =\left(q_{0}^{2}-|\mathbf{q}|^{2}\right) \mathbf{v}+2(\mathbf{q} \cdot \mathbf{v}) \mathbf{q}+2 q_{0}(\mathbf{q} \times \mathbf{v}) \\
& =\left(\left(q_{0}^{2}-\boldsymbol{q}^{T} \mathbf{q}\right) I_{3 \times 3}+2 \boldsymbol{q} \boldsymbol{q}^{T}+2 q_{0}\left[\boldsymbol{q}^{\times}\right]\right) \mathbf{v} \\
& \left.=\left(\begin{array}{ccc}
q_{1}^{2} & q_{1} q_{2} & q_{1} q_{3} \\
q_{1} q_{2} & q_{2}^{2} & q_{2} q_{3} \\
q_{1} q_{3} & q_{2} q_{3} & q_{3}^{2}
\end{array}\right]+2 q_{0}\left[\begin{array}{ccc}
0 & -q_{3} & q_{2} \\
q_{3} & 0 & -q_{1} \\
-q_{2} & q_{1} & 0
\end{array}\right]\right) \mathbf{v} \\
& =\left[\begin{array}{ccc}
\left.q_{0}^{2}+q_{1}^{2}-q_{2}^{2}-q_{3}^{2}\right) I_{3 \times 3}+2\left(q_{1} q_{2}-q_{0} q_{3}\right) & 2\left(q_{1} q_{3}+q_{0} q_{2}\right) \\
2\left(q_{1} q_{2}+q_{0} q_{3}\right) & q_{0}^{2}-q_{1}^{2}+q_{2}^{2}-q_{3}^{2} & 2\left(q_{2} q_{3}-q_{0} q_{1}\right) \\
2\left(q_{1} q_{3}-q_{0} q_{2}\right) & 2\left(q_{2} q_{3}+q_{0} q_{1}\right) & q_{0}^{2}-q_{1}^{2}-q_{2}^{2}+q_{3}^{2}
\end{array}\right] \mathbf{v} .
\end{aligned}
$$


Utilizando A.24 em A.28, pode-se escrever o operador $\mathbf{w}$ como

$$
\begin{aligned}
\mathbf{w} & =\left[\begin{array}{lll}
2\left(q_{0}^{2}+q_{1}^{2}\right)-1 & 2\left(q_{1} q_{2}-q_{0} q_{3}\right) & 2\left(q_{1} q_{3}+q_{0} q_{2}\right) \\
2\left(q_{1} q_{2}+q_{0} q_{3}\right) & 2\left(q_{0}^{2}+q_{2}^{2}\right)-1 & 2\left(q_{2} q_{3}-q_{0} q_{1}\right) \\
2\left(q_{1} q_{3}-q_{0} q_{2}\right) & 2\left(q_{2} q_{3}+q_{0} q_{1}\right) & 2\left(q_{0}^{2}+q_{3}^{2}\right)-1
\end{array}\right] \mathbf{v} \\
& =Q \mathbf{v} .
\end{aligned}
$$

Um resultado equivalente pode ser obtido para

$$
\begin{aligned}
\mathbf{w} & =q^{*} \otimes\left[\begin{array}{l}
0 \\
\mathbf{v}
\end{array}\right] \otimes q \\
& =\left(\left(q_{0}^{2}-\boldsymbol{q}^{T} \boldsymbol{q}\right) I_{3 \times 3}+2 \boldsymbol{q} \boldsymbol{q}^{T}-2 q_{0}\left[\boldsymbol{q}^{\times}\right]\right) \mathbf{v} \\
& =\left[\begin{array}{ccc}
q_{0}^{2}+q_{1}^{2}-q_{2}^{2}-q_{3}^{2} & 2\left(q_{1} q_{2}+q_{0} q_{3}\right) & 2\left(q_{1} q_{3}-q_{0} q_{2}\right) \\
2\left(q_{1} q_{2}-q_{0} q_{3}\right) & q_{0}^{2}-q_{1}^{2}+q_{2}^{2}-q_{3}^{2} & 2\left(q_{2} q_{3}+q_{0} q_{1}\right) \\
2\left(q_{1} q_{3}+q_{0} q_{2}\right) & 2\left(q_{2} q_{3}-q_{0} q_{1}\right) & q_{0}^{2}-q_{1}^{2}-q_{2}^{2}+q_{3}^{2}
\end{array}\right] \\
& =\left[\begin{array}{lll}
2\left(q_{0}^{2}+q_{1}^{2}\right)-1 & 2\left(q_{1} q_{2}+q_{0} q_{3}\right) & 2\left(q_{1} q_{3}-q_{0} q_{2}\right) \\
2\left(q_{1} q_{2}-q_{0} q_{3}\right) & 2\left(q_{0}^{2}+q_{2}^{2}\right)-1 & 2\left(q_{2} q_{3}+q_{0} q_{1}\right) \\
2\left(q_{1} q_{3}+q_{0} q_{2}\right) & 2\left(q_{2} q_{3}-q_{0} q_{1}\right) & 2\left(q_{0}^{2}+q_{3}^{2}\right)-1
\end{array}\right] \\
& =Q^{T} \mathbf{v}
\end{aligned}
$$

\section{A.3 Conversão entre quatérnios e ângulos de Euler}

O operador quatérnio de rotação é equivalente à matriz de rotação para a sequência de ângulos de Euler aeroespacial $\psi, \theta$ e $\phi$ (ângulos de proa, de arfagem e de rolagem, respectivamente). Os quatérnios constituintes são:

$$
\begin{aligned}
& q_{z}=\cos \frac{\psi}{2}+\operatorname{sen} \frac{\psi}{2} \mathbf{k} \\
& q_{y}=\cos \frac{\theta}{2}+\operatorname{sen} \frac{\theta}{2} \mathbf{j} \mathrm{e} \\
& q_{x}=\cos \frac{\phi}{2}+\operatorname{sen} \frac{\phi}{2} \mathbf{i}
\end{aligned}
$$

assim

$$
q=q_{z} q_{y} q_{x}=q_{0}+q_{1} \mathbf{i}+q_{2} \mathbf{j}+q_{3} \mathbf{k},
$$


sendo

$$
\begin{aligned}
& q_{0}=\cos \frac{\psi}{2} \cos \frac{\theta}{2} \cos \frac{\phi}{2}+\operatorname{sen} \frac{\psi}{2} \operatorname{sen} \frac{\theta}{2} \operatorname{sen} \frac{\phi}{2}, \\
& q_{1}=\cos \frac{\psi}{2} \cos \frac{\theta}{2} \operatorname{sen} \frac{\phi}{2}-\operatorname{sen} \frac{\psi}{2} \operatorname{sen} \frac{\theta}{2} \cos \frac{\phi}{2}, \\
& q_{2}=\cos \frac{\psi}{2} \operatorname{sen} \frac{\theta}{2} \cos \frac{\phi}{2}+\operatorname{sen} \frac{\psi}{2} \cos \frac{\theta}{2} \operatorname{sen} \frac{\phi}{2} \mathrm{e} \\
& q_{3}=\operatorname{sen} \frac{\psi}{2} \cos \frac{\theta}{2} \cos \frac{\phi}{2}-\cos \frac{\psi}{2} \operatorname{sen} \frac{\theta}{2} \operatorname{sen} \frac{\phi}{2} .
\end{aligned}
$$

Vale ressaltar que a sequência ângulo/eixo aeroespacial, $(\psi, \theta, \phi) \rightarrow(z y x)$, é meramente uma das vinte sequências possíveis.

A matriz de rotação em termos de cossenos diretores, correspondente a rotação dos ângulos de Euler $(\psi, \theta, \phi)$, com convenção $(z y x)$ é

$$
\begin{aligned}
R & =[\text { rij] }] R_{\phi}^{x} R_{\theta}^{y} R_{\psi}^{z} \\
& =\left[\begin{array}{ccc}
\cos \psi \cos \theta & \operatorname{sen} \psi \cos \theta & -\operatorname{sen} \theta \\
\cos \psi \operatorname{sen} \theta \operatorname{sen} \phi-\operatorname{sen} \psi \cos \phi & \operatorname{sen} \psi \operatorname{sen} \theta \operatorname{sen} \phi+\cos \psi \cos \phi & \cos \theta \operatorname{sen} \phi \\
\cos \psi \operatorname{sen} \theta \cos \phi+\operatorname{sen} \psi \operatorname{sen} \phi & \operatorname{sen} \psi \operatorname{sen} \theta \cos \phi-\cos \psi \operatorname{sen} \phi & \cos \theta \cos \phi
\end{array}\right] \\
& =\left[\begin{array}{ccc}
q_{0}^{2}+q_{1}^{2}-q_{2}^{2}-q_{3}^{2} & 2\left(q_{1} q_{2}+q_{0} q_{3}\right) & 2\left(q_{1} q_{3}-q_{0} q_{2}\right) \\
2\left(q_{1} q_{2}-q_{0} q_{3}\right) & q_{0}^{2}-q_{1}^{2}+q_{2}^{2}-q_{3}^{2} & 2\left(q_{2} q_{3}+q_{0} q_{1}\right) \\
2\left(q_{1} q_{3}+q_{0} q_{2}\right) & 2\left(q_{2} q_{3}-q_{0} q_{1}\right) & q_{0}^{2}-q_{1}^{2}-q_{2}^{2}+q_{3}^{2}
\end{array}\right] \\
= & {\left[\begin{array}{ccc}
2\left(q_{0}^{2}+q_{1}^{2}\right)-1 & 2\left(q_{1} q_{2}+q_{0} q_{3}\right) & 2\left(q_{1} q_{3}-q_{0} q_{2}\right) \\
2\left(q_{1} q_{2}-q_{0} q_{3}\right) & 2\left(q_{0}^{2}+q_{2}^{2}\right)-1 & 2\left(q_{2} q_{3}+q_{0} q_{1}\right) \\
2\left(q_{1} q_{3}+q_{0} q_{2}\right) & 2\left(q_{2} q_{3}-q_{0} q_{1}\right) & 2\left(q_{0}^{2}+q_{3}^{2}\right)-1
\end{array}\right] }
\end{aligned}
$$

Para os ângulos de Euler tem-se

$$
\begin{aligned}
& \tan \psi=\frac{r_{12}}{r_{11}}=\frac{2\left(q_{1} q_{2}+q_{0} q_{3}\right)}{q_{0}^{2}+q_{1}^{2}-q_{2}^{2}-q_{3}^{2}}=\frac{2\left(q_{1} q_{2}+q_{0} q_{3}\right)}{2\left(q_{0}^{2}+q_{1}^{2}\right)-1} \\
& \operatorname{sen} \theta=-r_{13}=-2\left(q_{1} q_{3}-q_{0} q_{2}\right) \\
& \tan \phi=\frac{r_{23}}{r_{33}}=\frac{2\left(q_{2} q_{3}+q_{0} q_{1}\right)}{q_{0}^{2}-q_{1}^{2}-q_{2}^{2}+q_{3}^{2}}=\frac{2\left(q_{2} q_{3}+q_{0} q_{1}\right)}{2\left(q_{0}^{2}+q_{3}^{2}\right)-1}
\end{aligned}
$$




\section{A.4 Derivada do quatérnio}

Pode-se relacionar $q(t)$ e $q(t+\Delta t)$ como segue

$$
\begin{aligned}
q(t+\Delta t) & =q(t) \Delta r(t) \\
\text { sendo } \Delta r(t) & =\cos \frac{\Delta \alpha}{2}+\operatorname{sen} \frac{\Delta \alpha}{2} \boldsymbol{r}(t)
\end{aligned}
$$

o quatérnio de transição incremental. Seu ângulo de rotação é $\Delta \alpha$ sobre o eixo definido pelo vetor unitário $\boldsymbol{r}(t)$. Assumindo $\Delta t \rightarrow 0$, tem-se

$$
\begin{aligned}
\Delta r(t) & =\cos \frac{\Delta \alpha}{2}+\operatorname{sen} \frac{\Delta \alpha}{2} \boldsymbol{r}(t) \\
& =1+\frac{\Delta \alpha}{2} \boldsymbol{r}(t) .
\end{aligned}
$$

Então

$$
\begin{aligned}
q(t+\Delta t) & =q(t)\left(1+\frac{\Delta \alpha}{2} \boldsymbol{r}(t)\right) \\
q(t+\Delta t)-q(t) & =\frac{\Delta \alpha}{2}\left[\begin{array}{c}
0 \\
\boldsymbol{r}(t)
\end{array}\right]
\end{aligned}
$$

Dividindo por $\Delta t$ e utilizando limite, obtém-se

$$
\begin{aligned}
\dot{q}(t) & =\lim _{\Delta t \rightarrow 0} \frac{q(t+\Delta t)-q(t)}{\Delta t} \\
& =\lim _{\Delta t \rightarrow 0} \frac{q(t) \otimes\left[\begin{array}{c}
0 \\
\boldsymbol{r}(t)
\end{array}\right] \frac{\Delta \alpha}{2}}{\Delta t} \\
& =q(t) \otimes\left[\begin{array}{c}
0 \\
\boldsymbol{r}(t)
\end{array}\right] \dot{\alpha}(t)=\frac{1}{2} q(t) \otimes\left[\begin{array}{c}
0 \\
\boldsymbol{\omega}(t)
\end{array}\right],
\end{aligned}
$$

sendo $\boldsymbol{\omega}(t)=\dot{\alpha} \boldsymbol{r}(t)$ o vetor velocidade angular do quatérnio $\Delta_{r}$.

Na forma de matriz, a derivada do quatérnio pode ser escrita

$$
\dot{q}(t)=\frac{1}{2} q(t) \otimes\left[\begin{array}{c}
0 \\
\omega(t)
\end{array}\right]=\frac{1}{2}\left[\begin{array}{cccc}
0 & -\omega_{1} & -\omega_{2} & -\omega_{3} \\
\omega_{1} & 0 & \omega_{3} & -\omega_{2} \\
\omega_{2} & -\omega_{3} & 0 & \omega_{1} \\
\omega_{3} & \omega_{2} & -\omega_{1} & 0
\end{array}\right]\left[\begin{array}{l}
q_{0} \\
q_{1} \\
q_{2} \\
q_{3}
\end{array}\right]
$$




$$
\begin{aligned}
= & \frac{1}{2}\left[\begin{array}{cccc}
q_{0} & -q_{1} & -q_{2} & -q_{3} \\
q_{1} & q_{0} & -q_{3} & q_{2} \\
q_{2} & q_{3} & q_{0} & -q_{1} \\
q_{3} & -q_{2} & q_{1} & q_{0}
\end{array}\right]\left[\begin{array}{c}
0 \\
\omega_{1} \\
\omega_{2} \\
\omega_{3}
\end{array}\right] \\
= & \frac{1}{2}\left[\begin{array}{ccc}
-q_{1} & -q_{2} & -q_{3} \\
q_{0} & -q_{3} & q_{2} \\
q_{3} & q_{0} & -q_{1} \\
-q_{2} & q_{1} & q_{0}
\end{array}\right]\left[\begin{array}{l}
\omega_{1} \\
\omega_{2} \\
\omega_{3}
\end{array}\right] .
\end{aligned}
$$

Similarmente, para a derivada do conjugado, tem-se

$$
\begin{aligned}
q^{*}(t)=-\frac{1}{2}\left[\begin{array}{c}
0 \\
\boldsymbol{\omega}(t)
\end{array}\right] \otimes q^{*}(t)=\frac{1}{2}\left[\begin{array}{cccc}
0 & \omega_{1} & \omega_{2} & \omega_{3} \\
-\omega_{1} & 0 & \omega_{3} & -\omega_{2} \\
-\omega_{2} & -\omega_{3} & 0 & \omega_{1} \\
-\omega_{3} & \omega_{2} & -\omega_{1} & 0
\end{array}\right]\left[\begin{array}{c}
q_{0} \\
-q_{1} \\
-q_{2} \\
-q_{3}
\end{array}\right] \\
=\frac{1}{2}\left[\begin{array}{cccc}
q_{0} & -q_{1} & -q_{2} & -q_{3} \\
-q_{1} & -q_{0} & q_{3} & -q_{2} \\
-q_{2} & -q_{3} & -q_{0} & q_{1} \\
-q_{3} & q_{2} & -q_{1} & -q_{0}
\end{array}\right]\left[\begin{array}{c}
0 \\
\omega_{1} \\
\omega_{2} \\
\omega_{3}
\end{array}\right] \\
=\frac{1}{2}\left[\begin{array}{ccc}
-q_{1} & -q_{2} & -q_{3} \\
-q_{0} & q_{3} & -q_{2} \\
-q_{3} & -q_{0} & q_{1} \\
q_{2} & -q_{1} & -q_{0}
\end{array}\right]\left[\begin{array}{c}
\omega_{1} \\
\omega_{2} \\
\omega_{3}
\end{array}\right] .
\end{aligned}
$$

\section{A.5 Integração do quatérnio}

A abordagem do quatérnio usando um vetor de quatro parâmetros com uma restrição de norma unitária permite três graus de liberdade. Devido à restrição de norma unitária, os quatérnios são elementos de uma esfera unitária em quatro espaços. Essa esfera unitária não é um espaço vetorial Euclideano onde se pode aplicar as definições de adição de vetores e escalonamento. Portanto, deve-se tomar cuidado na integração de equações diferenciais de quatérnios. 
Como mostrado em [36], se a taxa de amostragem é alta o suficiente e precisa para considerar a taxa de rotação como constante sobre o intervalo de amostragem $T$, então é possível encontrar e implementar uma solução de forma fechada para a Equação (A.37), que pode ser reescrita como:

$$
\dot{q}(t)=\frac{1}{2} Q(t) q(t)
$$

Como pretende-se encontrar $q\left(t_{2}\right)$ para $q\left(t_{1}\right)$ conhecido e $Q(t)$ constante para $t \in\left(t_{1}, t_{2}\right]$, sendo $T=t_{2}-t_{1}$, então

$$
Q(t)=Q=\frac{1}{2}\left[\begin{array}{cccc}
0 & -\omega_{1} & -\omega_{2} & -\omega_{3} \\
\omega_{1} & 0 & \omega_{3} & -\omega_{2} \\
\omega_{2} & -\omega_{3} & 0 & \omega_{1} \\
\omega_{3} & \omega_{2} & -\omega_{1} & 0
\end{array}\right]
$$

Definindo o fator de integração como $e^{-\int_{t_{1}}^{t} Q(\tau) d \tau}$ e multiplicando-o do lado esquerdo de A.39) tem-se

$$
\begin{aligned}
e^{-\int_{t_{1}}^{t} Q(\tau) d \tau} \dot{q}(t)-e^{-\int_{t_{1}}^{t} Q(\tau) d \tau} Q q(t) & =0 \\
\frac{d}{d t}\left(e^{-\int_{t_{1}}^{t} Q(\tau) d \tau} q(t)\right) & =0 .
\end{aligned}
$$

Integrando ambos os lados sobre o intervalo, tem-se

$$
\begin{aligned}
e^{-\int_{t_{1}}^{t_{2}} Q(\tau) d \tau} q\left(t_{2}\right) & =e^{-\int_{t_{1}}^{t_{1}} Q(\tau) d \tau} q\left(t_{1}\right) \\
q\left(t_{2}\right) & =e^{\int_{t_{1}}^{t_{2}} Q(\tau) d \tau} q\left(t_{1}\right),
\end{aligned}
$$

sendo identificado que $\left(e^{-\int_{t_{1}}^{t} Q(\tau) d \tau}\right)^{-1}=e^{\int_{t_{1}}^{t} Q(\tau) d \tau}$.

Para escrever (A.42) de uma forma mais conveniente, define-se $\mathbf{w}=\left[\begin{array}{lll}W_{1} & W_{2} & W_{3}\end{array}\right]^{T} \mathrm{e}$

$$
W=\left[\begin{array}{cccc}
0 & -W_{1} & -W_{2} & -W_{3} \\
W_{1} & 0 & -W_{3} & W_{2} \\
W_{2} & W_{3} & 0 & -W_{1} \\
W_{3} & -W_{2} & W_{1} & 0
\end{array}\right]
$$

sendo $W_{1}=\frac{1}{2} \int_{t_{1}}^{t} \omega_{1}(\tau) d \tau, W_{2}=\frac{1}{2} \int_{t_{1}}^{t} \omega_{2}(\tau) d \tau, W_{3}=\frac{1}{2} \int_{t_{1}}^{t} \omega_{3}(\tau) d \tau$.

Com estas definições, a equação (A.42) é equivalente a

$$
q\left(t_{2}\right)=e^{\int_{t_{1}}^{t_{2}} Q(\tau) d \tau} q\left(t_{1}\right) .
$$


Expandindo $e^{W}$ usando séries de Taylor, tem-se

$$
\begin{aligned}
e^{W} & =I+W+\frac{W^{2}}{2 !}+\frac{W^{3}}{3 !}+\ldots \\
& =\left(I+\frac{W^{2}}{2 !}+\frac{\left(W^{2}\right)^{2}}{4 !}+\ldots\right)+W\left(I+\frac{W^{2}}{3 !}+\frac{\left(W^{2}\right)^{2}}{5 !}+\ldots\right) \\
& =\cos (\| \mathbf{w} \mid) I+\frac{\sin (\| \mathbf{w} \mid)}{\|\mathbf{w}\|} W .
\end{aligned}
$$

Como a dedução acima pode ser repetida para qualquer intervalo de duração $T$ para qual é assumido que a taxa angular constante é válida, tem-se a equação geral

$$
q\left(t_{k}\right)=\left(\cos (\| \mathbf{w} \mid) I+\frac{\sin (\|\mathbf{w}\|)}{\|\mathbf{w}\|} W\right) q\left(t_{k-1}\right) .
$$

sendo $\mathbf{w},\|\mathbf{w}\|$ e $W$ envolve a integral da taxa angular sobre o intervalo de amostragem $t \in$ $\left(t_{k-1}, t_{k}\right]$.

A solução é em forma fechada dada a suposição que $Q(t)$ é constante sobre o intervalo de tamanho $T$. E a solução preserva a norma pois o lado direito da equação (A.46) é igual a $\left\|q\left(t_{k-1}\right)\right\|$, conforme mostrado em [36]. 